\title{
Testing Trace-Element Distribution and the Zr-Based Thermometry of Accessory Rutile from Chromitite
}

\author{
Federica Zaccarini ${ }^{1, *}$, Giorgio Garuti ${ }^{1}$, George L. Luvizotto ${ }^{2}$, Yuri de Melo Portella ${ }^{3}$ and Athokpam K. Singh ${ }^{4}$ \\ 1 Department of Applied Geosciences and Geophysics, University of Leoben, Peter Tunner Str. 5, \\ A8700 Leoben, Austria; giorgio.garuti1945@gmail.com \\ 2 Department of Geology, São Paulo State University (UNESP), 24-A Avenue, 1515, Rio Claro 13506-900, Brazil; \\ george.luvizotto@unesp.br \\ 3 Petrobras-Petróleo Brasileiro S.A., Avenida Henrique Valadares 28, Centro, Rio de Janeiro 20231-030, Brazil; \\ yuri_portella@hotmail.com \\ 4 Wadia Institute of Himalayan Geology, 33, General Mahadev Singh Road, Dehradun 248001, India; \\ aksingh_wihg@rediffmail.com \\ * Correspondence: federica.zaccarini@unileoben.ac.at; Tel.: +43-3842-402-6202
}

Citation: Zaccarini, F.; Garuti, G.; Luvizotto, G.L.; de Melo Portella, Y.; Singh, A.K. Testing Trace-Element Distribution and the Zr-Based Thermometry of Accessory Rutile from Chromitite. Minerals 2021, 11, 661. https://doi.org/10.3390/ $\min 11070661$

Academic Editor: Shoji Arai

Received: 24 May 2021

Accepted: 19 June 2021

Published: 22 June 2021

Publisher's Note: MDPI stays neutral with regard to jurisdictional claims in published maps and institutional affiliations.

Copyright: (c) 2021 by the authors. Licensee MDPI, Basel, Switzerland. This article is an open access article distributed under the terms and conditions of the Creative Commons Attribution (CC BY) license (https:// creativecommons.org/licenses/by/ $4.0 /)$.
Abstract: Trace element distribution and Zr-in-rutile temperature have been investigated in accessory rutile from stratiform (UG2, Merensky Reef, Jacurici), podiform (Loma Peguera), and metamorphic chromitites in cratonic shields (Cedrolina, Nuasahi). Rutile from chromitite has typical finger-print of $\mathrm{Cr}-\mathrm{V}-\mathrm{Nb}-\mathrm{W}-\mathrm{Zr}$, whose relative abundance distinguishes magmatic from metamorphic chromitite. In magmatic deposits, rutile precipitates as an intercumulus phase, or forms by exsolution from chromite, between $870{ }^{\circ} \mathrm{C}$ and $540{ }^{\circ} \mathrm{C}$. The $\mathrm{Cr}-\mathrm{V}$ in rutile reflects the composition of chromite, both $\mathrm{Nb}$ and $\mathrm{Zr}$ are moderately enriched, and $\mathrm{W}$ is depleted, except for in Jacurici, where moderate $\mathrm{W}$ excess was a result of crustal contamination of the mafic magma. In metamorphic deposits, rutile forms by removal of Ti-Cr-V from chromite during metamorphism between $650{ }^{\circ} \mathrm{C}$ and $400{ }^{\circ} \mathrm{C}$, consistent with greenschist-amphibolite facies, and displays variable $\mathrm{Cr}-\mathrm{Nb}$, low $\mathrm{V}-\mathrm{Zr}$, and anomalous enrichment in $\mathrm{W}$ caused by reaction with felsic fluids emanating from granitoid intrusions. All deposits, except Cedrolina, contain Rutile+PGM composite grains $(<10 \mu \mathrm{m})$ locked in chromite, possibly representing relics of orthomagmatic assemblages. The high $\mathrm{Cr}-\mathrm{V}$ content and the distinctive $\mathrm{W}-\mathrm{Nb}-\mathrm{Zr}$ signature that typifies accessory rutile in chromitite provide a new pathfinder to trace the provenance of detrital rutile in placer deposits.

Keywords: rutile; trace elements; Zr-in-rutile thermometry; chromite deposits; magmatic crystallization; metamorphism

\section{Introduction}

Titanium was discovered by William Gregor in 1791, and since the second half of the twentieth century, it has become a basic commodity essential to several modern technologies in the metal-alloys, steel, and pigment industries [1]. Rutile, the most common tetragonal form of titanium dioxide $\left(\mathrm{TiO}_{2}\right)$, and ilmenite $\left(\mathrm{FeTiO}_{3}\right)$ are the principal natural sources for titanium recovery. Rutile is a relatively abundant mineral in the Earth's crust [2], occurring as an accessory phase in various igneous and high-grade metamorphic rocks, although half of the present day annual production comes from detrital sedimentary sources in shoreline and fluvial placer deposits.

Besides its indisputable economic importance, rutile plays an important role in geosciences as a powerful petrogenetic indicator [3]. Although characterized by a simple chemical composition, $\mathrm{TiO}_{2}$, rutile may carry a variety of trace elements replacing $\mathrm{Ti}$ in the crystal lattice. The extent of the substitution of some specific elements is an effective guide to petrology, geochronology, and geothermometry of the rutile-bearing host rock, and can be used in some cases to identify the source rock of detrital rutile in sedimentary deposits [2-11]. 
It has been experimentally demonstrated [12] that the solubility of $\mathrm{ZrO}_{2}$ in rutile is temperature-dependent, particularly when rutile is properly buffered with zircon $\left(\mathrm{ZrSiO}_{4}\right)$ and silica $\left(\mathrm{SiO}_{2}\right)$. Furthermore, recent investigations have shown that the $\mathrm{Zr}$-based thermometer can be successfully applied to rutile from silica-free, ultramafic rocks such as chromitites $[13,14]$, since the obtained temperatures were in agreement with the geological setting and the temperatures calculated with other geothermometers. Before and after these contributions, accessory rutile had been reported from a number of chromitite occurrences [15-22]. However, detailed trace-element systematic has been provided only for single chromite deposits of Brazil and India $[13,23]$. This contribution presents the results of the first systematic study of the distribution of $\mathrm{Zr}$ and other trace elements in accessory rutile from chromitite of different ages and geological settings. The analyzed samples represent chromitite associated with high-grade metamorphic terranes of India and Brazil mentioned above, stratiform chromitite from continental layered intrusions of South Africa and Brazil, and one podiform chromitite from the Caribbean ophiolite belt of the Dominican Republic.

The main objectives were to (1) characterize the trace-element finger-print of rutile derived from chromitites with different magmatic and post-magmatic histories, (2) test the validity of the Zr-in-rutile thermometer applied to silica under-saturated rocks composed mainly of chromite, (3) evaluate the role of metamorphism in the re-distribution of $\mathrm{Zr}$ and other trace elements in chromitite-hosted rutile, and (4) discuss rutile-chromite relationships (texture, composition) and provide further argument to the debate on the origin of accessory rutile in chromitite: a primary magmatic phase, a product of subsolidus exsolution from the host chromite during magmatic cooling, or a result of regional metamorphism?

\section{Methodology}

Rutile was previously located on thin sections and polished blocks by reflected-light microscope and confirmed by Raman spectroscopy. Afterwards, electron microprobe analyses of chromite and rutile were carried out at the Eugen F. Stumpfl laboratory (Leoben University, Leoben, Austria), using a Jeol JXA 8200 Superprobe (Jeol, Tokyo, Japan). Wavelength dispersive system (WDS) was used for quantitative analyses. Rutile grains less than $10 \mu \mathrm{m}$ in size were qualitatively analyzed by energy dispersive system (EDS). The same instrument was used to obtain electron images.

Chromite was analyzed with $15 \mathrm{kV}$ accelerating voltage and $10 \mathrm{nA}$ beam current. All the elements were analyzed using the $\mathrm{K} \alpha$ line, and were calibrated on natural chromite, rhodonite, ilmenite, kaersutite, and olivine. The following diffracting crystals were used: TAP for Mg and Al; PETJ for Si; and LIFH for Ti, V, Cr, Mn, and Fe. Counting times of 20 and $10 \mathrm{~s}$ were used on peak and backgrounds, respectively. The amount of $\mathrm{Fe}^{3+}$ in chromite was calculated assuming the ideal spinel stoichiometry.

Preliminary electron microprobe analysis of accessory rutile indicated the systematic occurrence of trace amounts of $\mathrm{Mg}, \mathrm{Al}, \mathrm{V}, \mathrm{Cr}, \mathrm{Mn}, \mathrm{Fe}, \mathrm{Zr}, \mathrm{Nb}$, and $\mathrm{W}$. Qualitative analyses failed in finding other elements commonly reported from terrestrial rutiles (e.g., $\mathrm{Hf}, \mathrm{Ta}$, Th). Since most of the recorded metals are among the major constituents of the host chromite, and the analyses were performed in situ, special attention was paid to avoid spurious fluorescence from the matrix and unwanted interferences between analytical lines. Rutile was analyzed with $20 \mathrm{kV}$ accelerating voltage and $30 \mathrm{nA}$ beam current. The counting times were increased up to $60 \mathrm{~s}$ and $30 \mathrm{~s}$ on peak and backgrounds, respectively. The $\mathrm{K} \alpha$ line was used for $\mathrm{Mg}, \mathrm{Al}, \mathrm{Ti}, \mathrm{V}, \mathrm{Cr}, \mathrm{Mn}$, and $\mathrm{Fe}$, and the $\mathrm{L} \alpha$ line for $\mathrm{Nb}, \mathrm{Zr}$ and $\mathrm{W}$. The following standards were selected: natural olivine, chromite, rutile, rhodonite, magnetite and zircon, synthetic metallic $\mathrm{Nb}, \mathrm{V}$, and $\mathrm{W}$. The TAP crystal was used for $\mathrm{Mg}$ and $\mathrm{Al}$; the PETJ for $\mathrm{Nb}, \mathrm{Mn}$, and $\mathrm{Zr}$; and the LIFH for $\mathrm{Cr}, \mathrm{Ti}, \mathrm{W}, \mathrm{Fe}$, and $\mathrm{V}$. The detection limits of elements occurring as trace concentrations were automatically calculated by the Jeol software as follows: $\mathrm{Mg}, \mathrm{Al}, \mathrm{V}, \mathrm{Cr}, \mathrm{Zr}$, and $\mathrm{Fe}=50 \mathrm{ppm} ; \mathrm{Nb}, \mathrm{Ti}$, and $\mathrm{Mn}=100 \mathrm{ppm}$; and $\mathrm{W}=150 \mathrm{ppm}$. The same instrument was used to obtain the Back-Scattered Electron (BSE) images. Monitoring the accuracy and precision of several tens of analyses carried out under 
the abovementioned analytical conditions, it was concluded that only rutile grains larger than $10 \mu \mathrm{m}$ yielded reliable results, free of fluorescence effects and chemical contamination from the chromite host.

\section{Geological Setting and Petrographic Notes}

In order to provide a statistically significant collection of data, only chromitites containing high proportion of accessory rutile were considered in this work (Table 1). Podiform chromitites in ophiolitic mantle tectonite from supra-subduction-zone (SSZ) were found to be unsuitable due to the absence or scarcity of accessory rutile.

Table 1. Mafic-Ultramafic complexes host of rutile-bearing chromitite and sample description.

\begin{tabular}{|c|c|c|c|c|}
\hline $\begin{array}{l}\text { Complex/Tectonic } \\
\text { Setting/Deposit }\end{array}$ & Sample & Type/Size of Deposit & Ore Type/Alteration & Host Rock \\
\hline \multicolumn{5}{|c|}{$\begin{array}{l}\text { UNMETAMORPHOSED } \\
\text { Bushveld Complex (South Africa): Continental Layered Intrusion } \\
\text { (Paleoproterozoic) } \\
\text { W. Bushweld, Rustenburg underground section }\end{array}$} \\
\hline \multirow[t]{2}{*}{ UG2 chromitite layer } & UG2-A & $\begin{array}{l}\text { chromitite layer } 0.75 \mathrm{~m} \\
\text { thick top }\end{array}$ & Massive & pyroxenite, anorthosite \\
\hline & UG2-B & $\begin{array}{l}\text { chromitite layer } 0.75 \mathrm{~m} \\
\text { thick middle }\end{array}$ & Massive & pyroxenite, anorthosite \\
\hline \multirow[t]{2}{*}{$\begin{array}{c}\text { Merensky Reef } \\
\text { sulfide-PGE deposit }\end{array}$} & MR1 & $\begin{array}{l}\text { reef top chromite-seam } \\
0.3-0.8 \mathrm{~cm} \text { thick }\end{array}$ & $\begin{array}{l}\text { Massive to } \\
\text { disseminated }\end{array}$ & $\begin{array}{c}\text { pegmatoidal, feldspatic } \\
\text { pyroxenite }\end{array}$ \\
\hline & MR2 & $\begin{array}{c}\text { reef bottom } \\
\text { chromite-seam } 0.5-1.5 \\
\text { cm thick }\end{array}$ & Massive & $\begin{array}{c}\text { pegmatoidal, feldspatic } \\
\text { pyroxenite }\end{array}$ \\
\hline \multicolumn{5}{|c|}{$\begin{array}{l}\text { Jacurici Complex (Bahia State, Brazil): Intracratonic mafic-ultramafic sill } \\
\text { (Paleoproterozoic) }\end{array}$} \\
\hline $\begin{array}{l}\text { Ipuera Socò Prospect, } \\
\text { drill core S1-1 } 45^{\circ}\end{array}$ & JC135 & $\begin{array}{l}\text { chromitite layer } 1.7 \mathrm{~m} \\
\text { thick }\end{array}$ & Massive, altered rims & serpentinized dunite \\
\hline \multirow{2}{*}{$\begin{array}{l}\text { Varzea do Macaco-Teiù, } \\
\text { drill coreT1-5 } 45^{\circ}\end{array}$} & JC148 & $\begin{array}{l}\text { chromitite layer } 4.5 \mathrm{~m} \\
\text { thick, top }\end{array}$ & Massive, altered rims & $\begin{array}{l}\text { serpentinite }+ \\
\text { carbonate vein }\end{array}$ \\
\hline & JC150 & $\begin{array}{l}\text { chromitite layer } 4.5 \mathrm{~m} \\
\text { thick, middle }\end{array}$ & Massive, altered rims & $\begin{array}{l}\text { serpentinite }+ \\
\text { carbonate vein }\end{array}$ \\
\hline \multicolumn{5}{|c|}{$\begin{array}{l}\text { Loma Caribe Peridotite Massif (Dominican Republic): Intraoceanic Mantle } \\
\text { Tectonite Plume (Mesozoic) }\end{array}$} \\
\hline \multirow[t]{2}{*}{$\begin{array}{l}\text { Loma Peguera Nickel } \\
\text { mine (Bonao District) }\end{array}$} & LP4 & $\begin{array}{l}\text { chromitite pod } 0.30 \mathrm{~m} \\
\text { thick }\end{array}$ & Massive, altered rims & $\begin{array}{l}\text { laterite weathered } \\
\text { harzburgite }\end{array}$ \\
\hline & LP6 & $\begin{array}{l}\text { chromitite pod } 0.15 \mathrm{~m} \\
\text { thick }\end{array}$ & Massive, altered rims & $\begin{array}{l}\text { laterite weathered } \\
\text { harzburgite }\end{array}$ \\
\hline \multicolumn{5}{|c|}{$\begin{array}{l}\text { METAMORPHOSED } \\
\text { Nuasahi Complex (Orissa, Eastern India): Intracratonic Layered Intrusion } \\
\text { (Archean) }\end{array}$} \\
\hline $\begin{array}{l}\text { S. Nuasahi, hanging } \\
\text { wall Breccia Zone }\end{array}$ & NS9 & $\begin{array}{l}\text { chromitite fragment in } \\
\text { gabbro matrix }\end{array}$ & Massive altered & serpentinite \\
\hline \multicolumn{5}{|c|}{$\begin{array}{l}\text { Cedrolina Complex (Goias state, Brazil): Pilar de Goiás greenstone belt } \\
\text { (Paleoproterozoic) }\end{array}$} \\
\hline \multirow{5}{*}{$\begin{array}{l}\text { Cedrolina prospect, } \\
\text { detrital blocks }\end{array}$} & CD100 & $\begin{array}{l}\text { nudular chromitite } \\
\qquad(<0.5 \mathrm{~cm})\end{array}$ & Strongly weathered & talc-chlorite schist \\
\hline & CD105C & $\begin{array}{l}\text { brecciated nodular } \\
\text { chromitite }(<1.0 \mathrm{~cm})\end{array}$ & Moderately weathered & talc-chlorite schist \\
\hline & CD106D & $\begin{array}{l}\text { nudular chromitite } \\
\qquad(<1.5 \mathrm{~cm})\end{array}$ & Slightly altered & talc-chlorite schist \\
\hline & CD143 & & Slightly altered & talc-chlorite schist \\
\hline & CD47A3 & $\begin{array}{l}\text { brecciated nodular } \\
\text { chromitite }(<1.0 \mathrm{~cm})\end{array}$ & Intensely weathered & talc-chlorite schist \\
\hline
\end{tabular}


Three of the investigated deposits represent unmetamorphosed, magmatic chromitite deposited in distinctive geological settings and ages. They are Paleoproterozoic stratiform deposits associated with the layered intrusions of Bushveld (Transvaal Province, South Africa), the ultramafic-mafic sill of Jacurici (Bahia State, Brazil), and a Mesozoic podiform chromitite of Loma Peguera in the Loma Caribe ophiolite massif (Dominican Republic). Both Bushveld and Jacurici complexes intrude high-grade metamorphic terranes; however, texture, mineralogy, and chemical variations proper of cumulate rocks are generally well preserved, suggesting that regional metamorphism was not effective in these cases. The podiform chromitite of Loma Peguera is unaffected by high-grade regional metamorphism, but nonetheless the host peridotite shows widespread low-temperature serpentinization typical of sub-oceanic upper mantle, and lateritic weathering under supergene conditions.

Two of the examined chromite deposits are examples of chromitites involved in highgrade regional metamorphism, following primary magmatic deposition. The Nuasahi chromite deposit consists of a strongly deformed and metamorphosed layered sill associated with the Archaean cratonic shield of Singhbhum (Orissa province, Eastern India). The Cedrolina chromitite is located in the Archean-Paleoproterozoic greenstone belt of Pilar de Goiás (Central Brazil). The amphibolite facies metamorphic overprint of the area, metasomatic reactions with exotic fluids, and poly-cyclical tectonic deformations have prevented the identification of the original chromite magmatic affinity (podiform or stratiform).

\subsection{Bushveld Layered Intrusion (UG2 Chromitite Layer, Merensky Reef)}

The Bushveld Complex, located in the Transvaal Province of South Africa, is the largest mafic-ultramafic layered intrusion in the world. It consists of a $9 \mathrm{~km}$ thick sequence of layered mafic and ultramafic cumulates that hosts, in specific mineralized reefs, world-class deposits of chromium, platinum group elements (PGE), nickel, copper, and vanadium. Despite ages as old as $2.06 \mathrm{Ga}$, magmatic features are well preserved, showing minimal deformation and metamorphic effects [24,25].

The occurrence of accessory rutile in the chromitites was documented by various authors (e.g., [14-16,19-21]). Analytical data have been provided for rutile in pyroxenite from various localities of the UG2 and Merensky Reef units [14]. In this work, we analyzed rutile from massive chromitite collected along the Rustenburg Section of the Rustenburg Platinum Mine during the Third International Platinum Symposium, Pretoria, July 1981. They represent the UG2 chromitite layer and the chromite seams associated with the Ni-Cu-PGE deposit of Merensky Reef (UG2 and MR labels).

The UG2 is one of the uppermost chromitite layers in the Critical Zone of the Bushveld Complex and contains the largest single PGE resource in the world, while $\mathrm{Cr}$ is a byproduct [25-27]. In the Rustemburg Section, the UG2 chromitite is about $0.75 \mathrm{~m}$ thick and is located below the Merensky Reef, at an estimated stratigraphic distance of about $120 \mathrm{~m}$ [26]. The examined UG2 samples consist of a massive 60 to 90 modal percent chromite showing a granular texture derived from accumulation of euhedral to subhedral chromite variable in size from few microns up to $1 \mathrm{~mm}$. Internal layering is marked by alternating zones of massive chromite with zones of high-grade disseminated chromite with increasing modal percent of interstitial silicate mainly composed of pyroxene and plagioclase (Figure 1A).

In the Rustemburg Section, the Merensky Reef consists of a pegmatoidal feldspatic pyroxenite of about $0.3 \mathrm{~m}$ average thickness, densely spotted with $\mathrm{Ni}-\mathrm{Cu}$ magmatic sulfides and grains of platinum-group minerals (PGM). The Reef is bounded by two chromite layers, about $0.2-2 \mathrm{~cm}$ thick, marking the top and the bottom contacts with feldspatic pyroxenite hangingwall and spotted anorthosite footwall, respectively. 

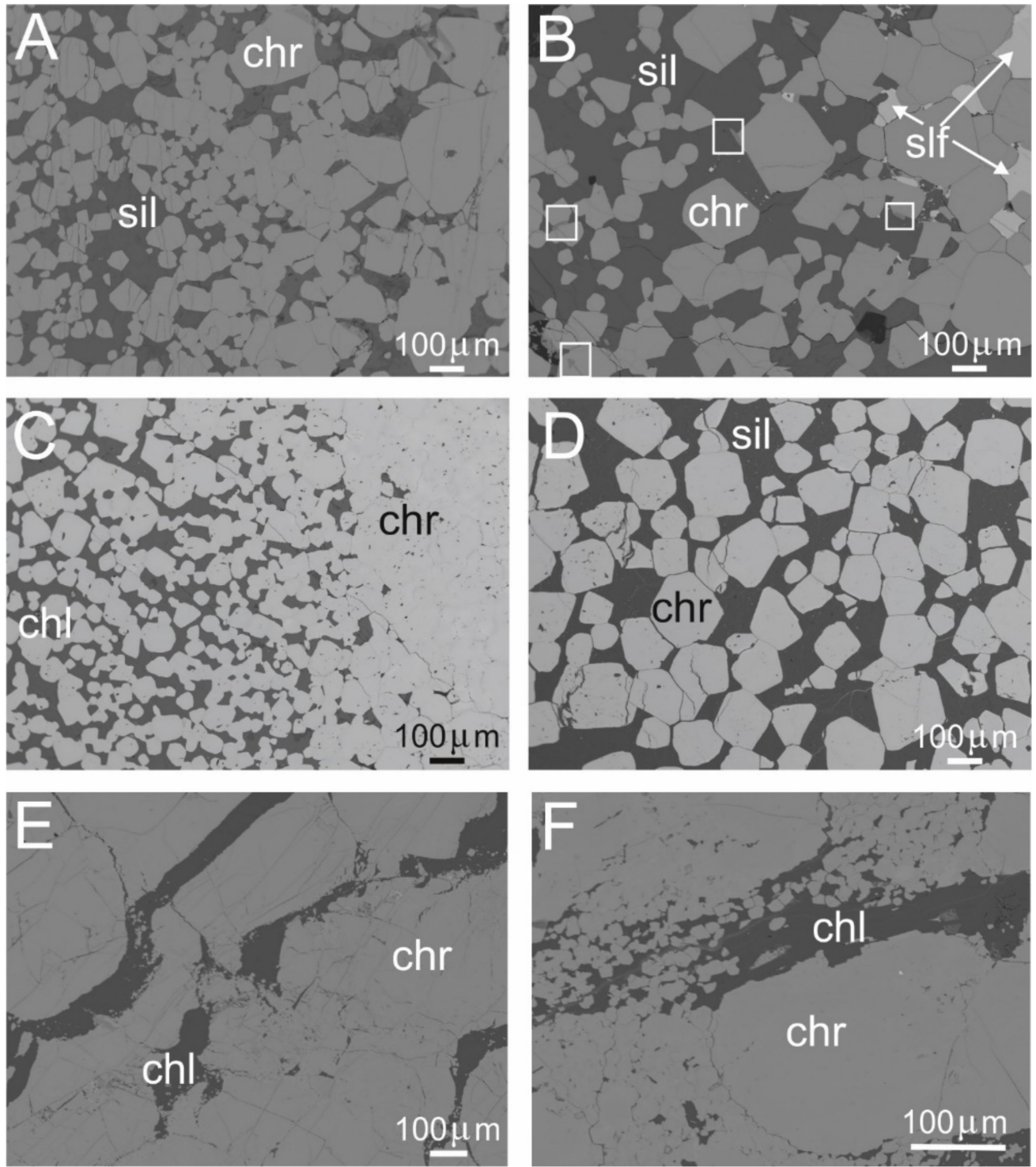

Figure 1. Back-scattered electron (BSE) images of chromite textures in unmetamorphic magmatic deposits. Cumulus textures showing transition from massive to high-grade disseminated chromite in UG2 (A), Merensky Reef (B), and Jacurici (sample JC96) (C). Equigranular cumulus chromite from Jacurici (sample JC150) (D). Massive fractured chromitite (E) and Massive chromitite with a cumulitic-like texture from Loma Peguera (sample LP4) (F). Boxes in B indicate position of rutile grains. Abbreviations: chr, chromite; sil, silicates; chl, chlorite; slf, sulfides.

Our chromitite samples consist of euhedral and subhedral grains, up to $1 \mathrm{~mm}$ in size, arranged in a typical cumulus texture (Figure 1B). Chromite grains are completely fresh and do not show any sign of alteration; however, the associated silicate matrix, mainly coarse pyroxene and plagioclase, contains minor amounts of hydrous minerals (actinolite, micas, talc, chlorite, and a serpentine subgroup phases) that have been recognized as a result of deuteric reaction with late-stage hydrous melt during solidification of the Merensky Reef [28-30].

\subsection{Jacurici Mafic-Ultramafic Sill (São Francisco Craton)}

The Jacurici Complex consists of a suite of ultramafic bodies intruding ArchaeanPaleoproterozoic terranes of the São Francisco Craton in the NE of Bahia State, Brazil [31-33]. According to zircon $\mathrm{U}-\mathrm{Pb}$ data, the crystallization age of the Jacurici Complex can be placed at about $2085 \mathrm{Ma}$ [34]. Structural reconstruction indicates that the ultramafic bodies of 
Jacurici represent dismembered segments of a single mafic-ultramafic layered sill, having an estimated thickness of about $300 \mathrm{~m}$, and extending laterally over about $100 \mathrm{~km}$ [35-37].

Ideal stratigraphy comprises a cumulus sequence of dunite, harzburgite, pyroxenite, and gabbro norite, with granulitic gneisses at the footwall, and calc-silicate marble at the hanging-wall. Despite its age and tectonic deformation, the Jacurici mafic-ultramafic sill does not appear to have suffered the high-grade metamorphism that affects the host rocks of the São Francisco Craton. However, the presence of partially melted xenoliths of carbonate country rocks and geochemical isotopic data support significant contamination of the mafic magma with crust-compatible lithophile elements [37].

A huge chromitite layer up to 2-8 $\mathrm{m}$ thick is found in the upper part of the ultramafic section (dunite, harzburgite), representing the largest chromite deposit in Brazil. The chromitite is generally massive and typical cumulus textures are common, showing decreasing grain size-grading, passing from massive to disseminated ore (Figure 1C), or locally characterized by accumulation of equigranular euhedral crystals (Figure 1D). Chromite is always well preserved, devoid of deformation and alteration, whilst most of the interstitial silicates (olivine, orthopyroxene) were transformed into chlorite by low temperature alteration. The altered silicate matrix contains scattered crystals of zircon up to $100 \mu \mathrm{m}$ in size. Accessory rutile in the chromitite of the Jacurici Complex has been reported by [22] and interpreted as an intercumulus mineral. Three samples of massive chromitite (JC label) from boreholes at the localities of Ipuera/Socò and Varzea do Macaco/Teiù were found to contain accessory rutile.

\subsection{Loma Peguera (Loma Caribe Ophiolite)}

The $100 \mathrm{~km}$ long peridotite belt of Loma Caribe in the Central Cordillera of the Dominican Republic is one of the ophiolite-related, ultramafic massifs exposed along the northern margin of the Caribbean Plate [38]. Unlike most ophiolite complexes worldwide, the Loma Caribe ophiolite is exclusively composed of harzburgitic mantle tectonite, with subordinate dunite and lherzolite, but lacks the other elements typical of ophiolites, i.e., the cumulus transition zone, sheeted dyke complex, pillow lavas, and pelagic sediments. According to the recently proposed geodynamic classification of ophiolites [39], the Loma Caribe peridotite belt may represent a rare case of mantle-plume emplaced in the oceanic plateau of the Mesozoic Caribbean basin [40-42].

Prolonged exposition to supergene alteration and weathering generated a thick layer of laterite on the top of the ultramafic rocks, leading to economic deposits of silicate$\mathrm{Ni}[43,44]$. In the mid1990s, mining exploration for Ni-laterite revealed the presence of small pods and lenses of chromitite $(<2 \mathrm{~m})$ randomly distributed within small masses of serpentinized dunite, in the area of Loma Peguera.

Two samples labelled LP were collected from the chromitites exposed at the outcrops visited during the field trip in the framework of the 18va Conferencia Geologica del Caribe in 2008. The samples consist of massive ore with less than $10 \%$ interstitial silicate, locally affected by fracturing and stretching (Figure 1E). Despite widespread brittle deformation, the chromite is generally fresh, and cumulus-like texture is observed in micro-domains consisting of tiny polygonal crystals in a silicate matrix mostly composed of chlorite and minor serpentine (Figure 1F). Several minute rutile crystals up to $20 \mu \mathrm{m}$ in size were observed in between or partially included in chromite grains.

\subsection{Nuasahi Layered Complex (Singhbhum Craton)}

The Nuasahi Complex, North Orissa Province, is situated in the southeastern part of the Singhbhum craton in the Eastern Indian Shield. The craton contains only a small proportion of relatively young $(2.1 \mathrm{Ga})$ Paleoproterozoic terranes, while the great bulk is composed of Archaean (3.5-3.1 Ga) formations characterized by high-grade metamorphic overprint. They comprise, in decreasing order of age, small fragments of an old supracrustal suite $(3.5 \mathrm{Ga})$, granite-gneiss batholits and tonalite-granodiorite intrusions $(3.5-3.1 \mathrm{Ga})$, greenstone belts $(>3.1 \mathrm{Ga}$ ) containing banded iron formation (BIF)-type ore deposits, and 
sill-like mafic-ultramafic intrusions with chromite-PGE deposits, including the Nuasahi Complex (3.3-3.1 Ga) [23,45-47].

The Nuasahi Complex forms an N-S elongated body extending over about $60 \mathrm{~km}$ NE of the city of Sukinda. A W-E cross section of the Nuasahi mining area [48] shows a $\sim 500 \mathrm{~m}$ thick ultramafic zone composed of enstatitite, olivine orthopyroxenite, dunite with chromitite layers, and orthopyroxenite, limited to the $\mathrm{E}$ by a several decametres-thick zone of tectonic breccia that marks the transition to the Suite-2 gabbroic formation. Structural data indicate that the breccia resulted from the gabbro intrusion [47] and consists of a gabbroic matrix engulfing angular to sub-rounded fragments of massive, and banded chromitite. The Breccia Zone is intruded by pegmatitic gabbro and quartz-diorite veins and displays widespread evidence of greenschist-amphibolite grade metamorphism [48]. The chromitite sample analyzed in this contribution (label NS9) comes from a strongly deformed area of the Suite-2 gabbro, in the hanging-wall of the Breccia Zone, exposed to the south of the Nuasahi mining area [23].

Under the microscope, the chromitite is massive to disseminated and composed of about $70 \%$ chromite by volume. Chromite grains appear as irregular fragments with jagged boundaries and extremely variable size, from a few hundred microns to more than one millimeter (Figure 2A-C), and interpreted as a result of recrystallization [23]. The interstitial silicate matrix essentially consists of enstatite and minor Mg-hornblende (Figure 2B), with minimal formation of low-temperature hydrous silicates, i.e., chlorite, suggesting that the metamorphic grade was higher than hydrothermal. The presence of rutile in chromitites from Nuasahi and Singhbhum craton was previously reported by $[23,49]$.

\subsection{Cedrolina Ultramafic Body (Pilar de Goiás Greenstone Belt)}

The Pilar de Goiás greenstone belt is exposed in the Tocantins Province, State of Goiás, central Brazil. The belt consists of a volcano-sedimentary sequence metamorphosed under greenschist to amphibolite facies conditions. It is intruded by small bodies of albite-granite and affected by a multistage deformation during Paleoproterozoic and Neoproterozoic times [50].

The Cedrolina chromitite body is located within the lower stratigraphic units of the greenstone belt, where metakomatiites and metabasalts (talc-chlorite-schist, amphibolite and amphibole-schists) predominate over inter-bedded metasediments (metacherts, BIF). The chromitite has a tabular geometry of about $230 \mathrm{~m} \times 100 \mathrm{~m}$ and $1-2.4 \mathrm{~m}$ thickness, being in contact with micaschists to the SE, and amphibole rich BIF to the NW $[13,51]$. At the scale of hand samples, the chromitite consists of rounded nodules, up to $1 \mathrm{~cm}$ in diameter, embedded in a matrix of chlorite and talc, with a mean modal composition of $60 \%$ chromite, $25 \%$ talc, and $15 \%$ Cr-rich chlorite.

Under the microscope, the chromite nodules display a complex internal texture possibly resulting from coalescence of polygonal chromite crystals that still preserve sharp angular boundaries and triple junctions (Figure 2D). The nodules may be strongly fractured, with fissures mostly filled with chlorite. The borders may be extremely rugged, suggesting late corrosion or tectonic deformation, or smooth, with the nodules displaying less intense fissuring (Figure 2E). Different patterns of corrosion/deformation are seen causing dense riddling of the spinel body with microscopic holes (Figure 2F, left side) or developing minute fissures according to the cubic symmetry of spinel faces (Figure 2F, center and top).

Single crystals of chromite, variable in size from few microns up to $1 \mathrm{~mm}$ and, eventually, with hexagonal and octahedral shapes, have been also described [13,51], but are not shown in the pictures. According to these authors, the Cedrolina chromitite suffered intense metasomatism related to hydrothermal fluids emanated by albite-granite intrusions, which generated a complex suite of exotic accessory minerals such as monazite-Ce [52], uraninite, thorianite, and zircon. The samples studied here (CD label) are taken from the same collection of [13]. 

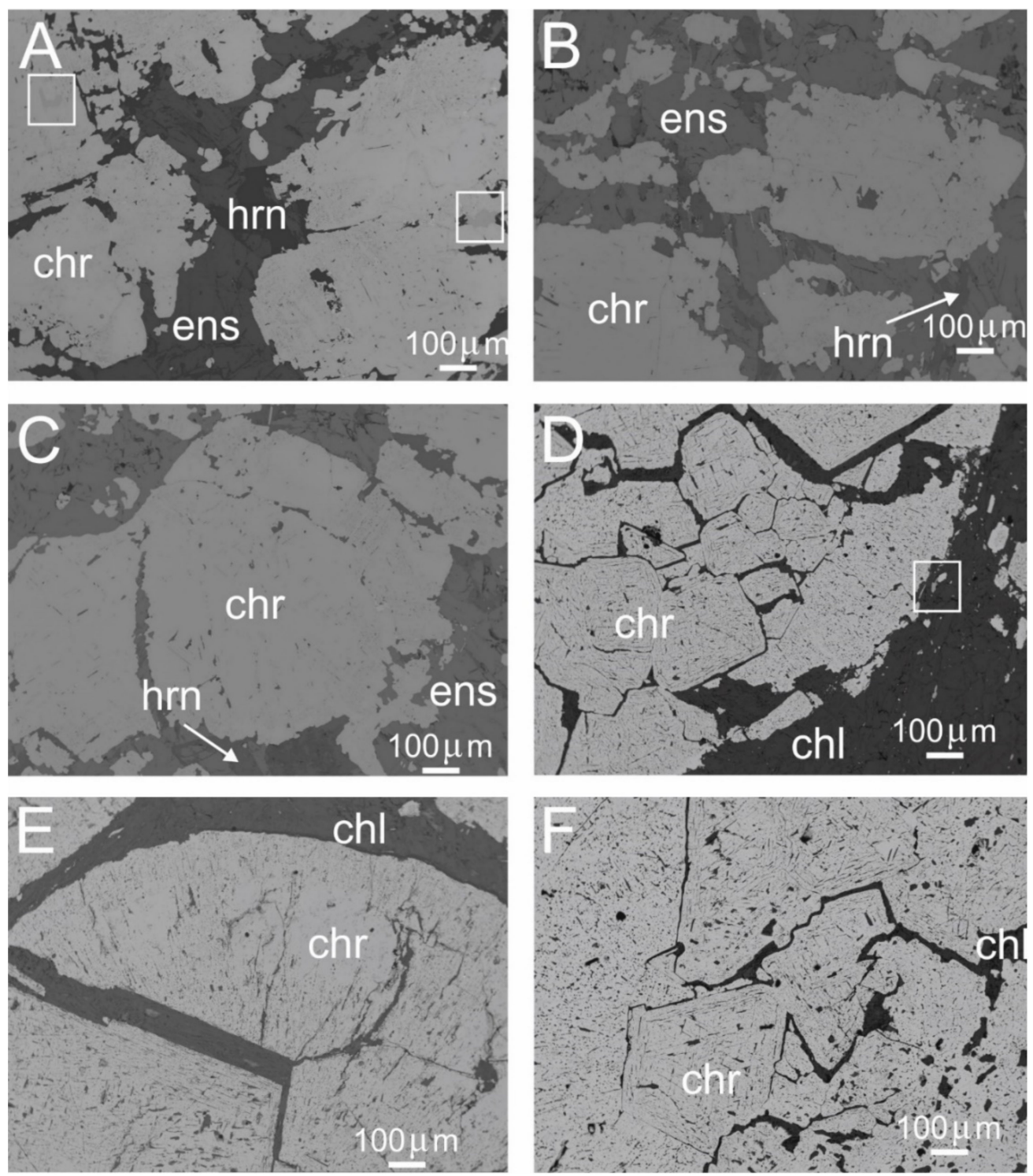

Figure 2. BSE images of chromite texture in metamorphic deposits. Strongly recrystallized and partially altered chromite crystals from the chromite fragment in the Nuasahi Breccia Zone (sample NS9) (A-C). Internal texture of altered and fractured chromite nodules of Cedrolina showing polygonal chromite fragments with sharp angular boundaries and triple junctions (sample CD105C) (D). Large nodule displaying increasing alteration towards borders and crosscutting fractures (sample CD105B) (E,F). Different corrosion patterns in altered spinel involving development of minute fissures according to the cubic symmetry (center and top of picture) or dense riddling with microscopic holes (left side of picture) (sample CD105C) (F). Boxes in A and D indicate position of rutile grains. Abbreviations: ens, enstatite; hrn, hornblende; others as in Figure 1.

\section{Chromite Mineral Chemistry}

Representative compositions of chrome spinels from the rutile-bearing chromitite are listed in Table 2 and presented as relevant binary diagrams in Figure 3. Most compositions (UG2, MR, LP, CD) plot at relatively high $\mathrm{Cr} \#$ and $\mathrm{Fe}^{2} \#$ numbers, in the ranges $\mathrm{Cr} /(\mathrm{Cr}+\mathrm{Al})$ $=0.70-0.90$ and $\mathrm{Fe}^{2} /\left(\mathrm{Fe}^{2}+\mathrm{Mg}\right)=0.56-0.92$, respectively (Figure 3A). Chromitites from Jacurici and Nuasahi plot out of this field showing relatively low chromium numbers at low and intermediate iron numbers of $\mathrm{Fe}^{2} \#=0.43-0.48$ at Jacurici, and $\mathrm{Fe}^{2} \#=0.65-0.73$ at Nuasahi. Variation of $\mathrm{TiO}_{2}, \mathrm{Fe}_{2} \mathrm{O}_{3}$, and $\mathrm{V}_{2} \mathrm{O}_{3}$ as function of $\mathrm{Cr}_{2} \mathrm{O} 3 \mathrm{wt} \%$ define distinctive trends according to the various types of deposits (Figure 3B-D). 
Table 2. Selected microprobe analyses ( $w \mathrm{t} \%$ ) of chromite from rutile-bearing chromitites.

\begin{tabular}{|c|c|c|c|c|c|c|c|c|c|c|c|c|c|c|c|}
\hline Sample & $\mathrm{SiO}_{2}$ & $\mathrm{TiO}_{2}$ & $\mathrm{Al}_{2} \mathrm{O}_{3}$ & $\mathrm{FeO}$ & $\mathrm{Fe}_{2} \mathrm{O}_{3}$ & $\mathrm{MgO}$ & $\mathrm{MnO}$ & $\mathrm{Cr}_{2} \mathrm{O}_{3}$ & $\mathrm{~V}_{2} \mathrm{O}_{3}$ & Total & $\mathrm{Fe}^{2} \#$ & Cr\# & Al & $\mathrm{Fe}^{3}$ & $\mathrm{Cr}$ \\
\hline & \multicolumn{15}{|c|}{ Bushveld (UG2 chromitite layer) } \\
\hline UG2-1 & 0.00 & 0.89 & 11.70 & 24.68 & 14.71 & 6.03 & 0.39 & 41.16 & 0.34 & 99.89 & 0.70 & 0.70 & 0.470 & 0.38 & 1.11 \\
\hline UG2-2 & 0.02 & 0.97 & 11.55 & 24.74 & 14.17 & 6.05 & 0.28 & 41.56 & 0.48 & 99.82 & 0.70 & 0.71 & 0.465 & 0.36 & 1.12 \\
\hline UG2-3 & 0.01 & 1.14 & 11.32 & 24.44 & 13.88 & 6.21 & 0.35 & 41.75 & 0.39 & 99.49 & 0.69 & 0.71 & 0.457 & 0.36 & 1.13 \\
\hline UG2-4 & 0.00 & 1.19 & 11.35 & 25.49 & 14.31 & 5.78 & 0.38 & 41.77 & 0.44 & 100.70 & 0.71 & 0.71 & 0.454 & 0.37 & 1.12 \\
\hline UG2-5 & 0.00 & 1.17 & 11.62 & 24.59 & 13.70 & 6.29 & 0.39 & 42.01 & 0.41 & 100.18 & 0.69 & 0.71 & 0.465 & 0.35 & 1.13 \\
\hline UG2-6 & 0.03 & 1.42 & 10.62 & 25.21 & 14.41 & 6.05 & 0.38 & 42.12 & 0.38 & 100.62 & 0.70 & 0.73 & 0.426 & 0.37 & 1.13 \\
\hline UG2-7 & 0.01 & 0.78 & 12.39 & 24.82 & 14.08 & 6.23 & 0.41 & 42.19 & 0.45 & 101.36 & 0.69 & 0.70 & 0.489 & 0.35 & 1.12 \\
\hline UG2-8 & 0.01 & 1.03 & 10.64 & 24.80 & 14.99 & 6.07 & 0.34 & 42.31 & 0.39 & 100.59 & 0.70 & 0.73 & 0.427 & 0.38 & 1.14 \\
\hline UG2-9 & 0.01 & 1.35 & 10.44 & 25.06 & 14.31 & 6.03 & 0.33 & 42.35 & 0.40 & 100.29 & 0.70 & 0.73 & 0.420 & 0.37 & 1.14 \\
\hline UG2-10 & 0.02 & 1.13 & 11.48 & 25.58 & 13.74 & 5.83 & 0.34 & 42.40 & 0.45 & 100.97 & 0.71 & 0.71 & 0.458 & 0.35 & 1.13 \\
\hline UG2-11 & 0.01 & 1.22 & 11.61 & 24.90 & 12.87 & 6.16 & 0.40 & 42.52 & 0.45 & 100.14 & 0.69 & 0.71 & 0.465 & 0.33 & 1.14 \\
\hline UG2-12 & 0.02 & 1.18 & 11.70 & 25.03 & 12.94 & 6.14 & 0.46 & 42.76 & 0.40 & 100.63 & 0.70 & 0.71 & 0.466 & 0.33 & 1.14 \\
\hline UG2-13 & 0.00 & 1.10 & 11.62 & 25.13 & 12.87 & 6.07 & 0.34 & 43.18 & 0.41 & 100.72 & 0.70 & 0.71 & 0.463 & 0.33 & 1.15 \\
\hline UG2-14 & 0.00 & 1.03 & 11.57 & 24.91 & 13.21 & 5.90 & 0.29 & 41.95 & 0.41 & 99.27 & 0.70 & 0.71 & 0.468 & 0.34 & 1.14 \\
\hline UG2-15 & 0.03 & 1.23 & 11.13 & 25.08 & 14.06 & 5.90 & 0.38 & 41.61 & 0.43 & 99.85 & 0.70 & 0.71 & 0.449 & 0.36 & 1.13 \\
\hline UG2-16 & 0.03 & 1.08 & 11.30 & 25.51 & 14.61 & 5.90 & 0.33 & 42.08 & 0.46 & 101.30 & 0.71 & 0.71 & 0.450 & 0.37 & 1.12 \\
\hline UG2-17 & 0.07 & 0.96 & 11.80 & 24.48 & 13.13 & 6.01 & 0.41 & 41.41 & 0.42 & 98.68 & 0.70 & 0.70 & 0.479 & 0.34 & 1.13 \\
\hline UG2-18 & 0.01 & 1.03 & 11.63 & 25.12 & 14.06 & 5.95 & 0.39 & 41.90 & 0.43 & 100.53 & 0.70 & 0.71 & 0.465 & 0.36 & 1.12 \\
\hline UG2-19 & 0.00 & 0.88 & 11.68 & 25.18 & 14.00 & 5.90 & 0.38 & 42.45 & 0.43 & 100.89 & 0.71 & 0.71 & 0.465 & 0.36 & 1.14 \\
\hline UG2-20 & 0.00 & 1.36 & 10.66 & 24.91 & 14.39 & 5.95 & 0.42 & 41.45 & 0.43 & 99.57 & 0.70 & 0.72 & 0.432 & 0.37 & 1.13 \\
\hline \multirow[t]{2}{*}{$\begin{array}{c}\text { UG2- } \\
\text { Average }\end{array}$} & 0.01 & 1.11 & 11.39 & 24.98 & 13.92 & 6.02 & 0.37 & 42.05 & 0.42 & 100.28 & 0.70 & 0.71 & 0.46 & 0.36 & 1.13 \\
\hline & \multicolumn{15}{|c|}{ Bushveld (Merensky Reef) } \\
\hline MR1-1 & 0.02 & 2.71 & 4.93 & 31.87 & 16.75 & 1.85 & 0.40 & 40.94 & 0.90 & 100.37 & 0.91 & 0.85 & 0.211 & 0.46 & 1.18 \\
\hline MR1-2 & 0.01 & 1.54 & 5.40 & 31.05 & 18.14 & 1.52 & 0.47 & 40.53 & 0.83 & 99.50 & 0.92 & 0.83 & 0.234 & 0.50 & 1.18 \\
\hline MR1-3 & 0.01 & 1.91 & 5.36 & 31.96 & 18.10 & 1.49 & 0.47 & 41.08 & 0.82 & 101.19 & 0.92 & 0.84 & 0.228 & 0.49 & 1.17 \\
\hline MR1-4 & 0.04 & 2.10 & 5.06 & 31.84 & 17.46 & 1.69 & 0.38 & 41.60 & 0.91 & 101.08 & 0.91 & 0.85 & 0.215 & 0.47 & 1.19 \\
\hline MR1-5 & 0.03 & 1.68 & 5.15 & 31.16 & 17.94 & 1.73 & 0.47 & 41.69 & 0.86 & 100.71 & 0.91 & 0.84 & 0.220 & 0.49 & 1.19 \\
\hline MR2-1 & 0.03 & 3.25 & 9.24 & 28.71 & 14.48 & 4.50 & 0.33 & 37.95 & 0.54 & 99.03 & 0.78 & 0.73 & 0.384 & 0.38 & 1.06 \\
\hline MR2-2 & 0.01 & 3.23 & 9.27 & 29.02 & 14.80 & 4.42 & 0.43 & 38.45 & 0.52 & 100.16 & 0.79 & 0.74 & 0.381 & 0.39 & 1.06 \\
\hline MR2-3 & 0.02 & 2.30 & 9.20 & 27.96 & 15.56 & 4.35 & 0.37 & 38.72 & 0.54 & 99.02 & 0.78 & 0.74 & 0.382 & 0.41 & 1.08 \\
\hline MR2-4 & 0.00 & 2.90 & 9.41 & 28.72 & 14.73 & 4.44 & 0.32 & 38.71 & 0.51 & 99.75 & 0.78 & 0.73 & 0.388 & 0.39 & 1.07 \\
\hline MR2-5 & 0.00 & 3.33 & 9.33 & 29.11 & 15.02 & 4.39 & 0.36 & 37.64 & 0.56 & 99.74 & 0.79 & 0.73 & 0.385 & 0.40 & 1.04 \\
\hline MR2-6 & 0.05 & 2.64 & 9.14 & 28.40 & 16.29 & 4.45 & 0.35 & 37.87 & 0.54 & 99.73 & 0.78 & 0.74 & 0.377 & 0.43 & 1.05 \\
\hline MR2-7 & 0.00 & 3.04 & 8.88 & 29.08 & 15.58 & 4.26 & 0.37 & 38.31 & 0.52 & 100.04 & 0.79 & 0.74 & 0.366 & 0.41 & 1.06 \\
\hline MR2-8 & 0.78 & 2.70 & 9.27 & 28.48 & 16.25 & 5.11 & 0.56 & 36.92 & 0.56 & 100.62 & 0.76 & 0.73 & 0.376 & 0.42 & 1.01 \\
\hline MR2-9 & 0.03 & 2.81 & 8.07 & 29.11 & 16.53 & 3.86 & 0.41 & 38.12 & 0.62 & 99.56 & 0.81 & 0.76 & 0.337 & 0.44 & 1.07 \\
\hline MR2-10 & 0.06 & 2.71 & 8.29 & 28.90 & 15.51 & 4.06 & 0.35 & 39.17 & 0.60 & 99.65 & 0.80 & 0.76 & 0.345 & 0.41 & 1.09 \\
\hline MR2-11 & 0.06 & 3.13 & 7.68 & 29.57 & 16.11 & 3.98 & 0.34 & 38.99 & 0.58 & 100.45 & 0.81 & 0.77 & 0.318 & 0.43 & 1.08 \\
\hline MR2-12 & 0.00 & 2.43 & 7.57 & 28.41 & 17.57 & 3.92 & 0.34 & 38.13 & 0.62 & 98.99 & 0.80 & 0.77 & 0.319 & 0.47 & 1.08 \\
\hline MR2-13 & 0.00 & 3.14 & 7.78 & 29.07 & 16.42 & 3.98 & 0.39 & 37.82 & 0.64 & 99.23 & 0.80 & 0.77 & 0.326 & 0.44 & 1.06 \\
\hline MR2-14 & 0.00 & 3.19 & 8.03 & 29.19 & 16.14 & 4.01 & 0.33 & 37.73 & 0.63 & 99.25 & 0.80 & 0.76 & 0.336 & 0.43 & 1.06 \\
\hline MR2-15 & 0.02 & 2.62 & 8.08 & 28.93 & 15.68 & 3.91 & 0.39 & 39.34 & 0.62 & 99.59 & 0.81 & 0.77 & 0.337 & 0.42 & 1.10 \\
\hline \multirow[t]{2}{*}{ MR-Average } & 0.06 & 2.67 & 7.76 & 29.53 & 16.25 & 3.60 & 0.39 & 38.98 & 0.64 & 99.88 & 0.82 & 0.77 & 0.32 & 0.43 & 1.09 \\
\hline & \multicolumn{15}{|c|}{ Jacurici (ultramafic sill) } \\
\hline JC135-2 & 0.03 & 0.20 & 18.51 & 17.08 & 1.61 & 11.50 & 0.18 & 50.00 & 0.02 & 99.15 & 0.45 & 0.64 & 0.694 & 0.04 & 1.26 \\
\hline JC135-5 & 0.00 & 0.27 & 18.77 & 17.30 & 1.12 & 11.50 & 0.23 & 50.55 & 0.05 & 99.78 & 0.46 & 0.64 & 0.699 & 0.03 & 1.26 \\
\hline JC135-6 & 0.01 & 0.24 & 18.75 & 17.26 & 0.77 & 11.49 & 0.23 & 50.95 & 0.01 & 99.71 & 0.46 & 0.65 & 0.698 & 0.02 & 1.27 \\
\hline JC135-7 & 0.03 & 0.32 & 18.64 & 17.74 & 0.54 & 11.32 & 0.23 & 51.13 & 0.06 & 100.01 & 0.47 & 0.65 & 0.694 & 0.01 & 1.28 \\
\hline JC135-10 & 0.04 & 0.18 & 18.94 & 17.83 & 0.64 & 11.23 & 0.23 & 50.96 & 0.13 & 100.20 & 0.47 & 0.64 & 0.704 & 0.02 & 1.27 \\
\hline JC135-13 & 0.01 & 0.26 & 18.79 & 17.03 & 1.64 & 11.65 & 0.15 & 50.02 & 0.04 & 99.58 & 0.45 & 0.64 & 0.700 & 0.04 & 1.25 \\
\hline JC135-15 & 0.13 & 0.27 & 18.64 & 17.64 & 0.80 & 11.47 & 0.23 & 50.95 & 0.03 & 100.16 & 0.46 & 0.65 & 0.692 & 0.02 & 1.27 \\
\hline JC148-2 & 0.00 & 0.34 & 17.39 & 17.86 & 1.98 & 11.21 & 0.19 & 51.38 & 0.01 & 100.36 & 0.47 & 0.66 & 0.650 & 0.05 & 1.29 \\
\hline JC148-7 & 0.03 & 0.27 & 16.21 & 17.34 & 2.57 & 11.25 & 0.24 & 51.84 & 0.04 & 99.78 & 0.46 & 0.68 & 0.611 & 0.06 & 1.31 \\
\hline JC148-9 & 0.00 & 0.43 & 16.83 & 17.57 & 3.04 & 11.47 & 0.23 & 51.35 & 0.02 & 100.93 & 0.46 & 0.67 & 0.626 & 0.07 & 1.28 \\
\hline JC148-11 & 0.00 & 0.15 & 17.13 & 16.80 & 3.51 & 11.67 & 0.21 & 50.71 & 0.03 & 100.20 & 0.45 & 0.67 & 0.640 & 0.08 & 1.27 \\
\hline JC148-12 & 0.01 & 0.25 & 16.66 & 18.03 & 3.09 & 11.14 & 0.32 & 52.03 & 0.01 & 101.54 & 0.48 & 0.68 & 0.618 & 0.07 & 1.30 \\
\hline JC148-14 & 0.00 & 0.53 & 16.57 & 17.99 & 2.53 & 11.19 & 0.22 & 51.71 & 0.04 & 100.78 & 0.47 & 0.68 & 0.619 & 0.06 & 1.30 \\
\hline JC150-1 & 0.02 & 0.21 & 17.07 & 16.93 & 1.71 & 11.64 & 0.30 & 52.46 & 0.04 & 100.38 & 0.45 & 0.67 & 0.636 & 0.04 & 1.31 \\
\hline JC150-2 & 0.18 & 0.34 & 17.65 & 16.72 & 1.38 & 11.91 & 0.27 & 51.23 & 0.07 & 99.76 & 0.44 & 0.66 & 0.659 & 0.03 & 1.28 \\
\hline JC150-3 & 0.01 & 0.12 & 17.90 & 16.62 & 2.18 & 11.71 & 0.27 & 51.01 & 0.05 & 99.87 & 0.44 & 0.66 & 0.668 & 0.05 & 1.28 \\
\hline JC $150-4$ & 0.05 & 0.31 & 17.76 & 17.24 & 1.54 & 11.60 & 0.22 & 51.44 & 0.04 & 100.19 & 0.45 & 0.66 & 0.661 & 0.04 & 1.28 \\
\hline JC150-10 & 0.02 & 0.14 & 17.95 & 16.32 & 1.95 & 12.01 & 0.28 & 51.22 & 0.02 & 99.90 & 0.43 & 0.66 & 0.668 & 0.05 & 1.28 \\
\hline JC150-13 & 0.00 & 0.16 & 17.95 & 16.14 & 2.18 & 12.03 & 0.29 & 50.79 & 0.03 & 99.57 & 0.43 & 0.66 & 0.670 & 0.05 & 1.27 \\
\hline JC150-15 & 0.03 & 0.19 & 17.58 & 16.60 & 1.55 & 11.65 & 0.23 & 50.96 & 0.04 & 98.83 & 0.44 & 0.66 & 0.663 & 0.04 & 1.29 \\
\hline JC-Average & 0.03 & 0.26 & 17.78 & 17.20 & 1.82 & 11.53 & 0.24 & 51.13 & 0.04 & 100.04 & 0.46 & 0.66 & 0.66 & 0.04 & 1.28 \\
\hline
\end{tabular}


Table 2. Cont.

\begin{tabular}{|c|c|c|c|c|c|c|c|c|c|c|c|c|c|c|c|}
\hline Sample & $\mathrm{SiO}_{2}$ & $\mathrm{TiO}_{2}$ & $\mathrm{Al}_{2} \mathrm{O}_{3}$ & $\mathrm{FeO}$ & $\mathrm{Fe}_{2} \mathrm{O}_{3}$ & $\mathrm{MgO}$ & $\mathrm{MnO}$ & $\mathrm{Cr}_{2} \mathrm{O}_{3}$ & $\mathrm{~V}_{2} \mathrm{O}_{3}$ & Total & $\mathrm{Fe}^{2} \#$ & Cr\# & Al & $\mathrm{Fe}^{3}$ & $\mathrm{Cr}$ \\
\hline & \multicolumn{15}{|c|}{ Loma Peguera (chromitite pod in mantle tectonite) } \\
\hline LP4-1 & 0.00 & 0.76 & 9.68 & 20.91 & 6.74 & 8.25 & 0.39 & 52.31 & 0.18 & 99.22 & 0.59 & 0.78 & 0.387 & 0.17 & 1.40 \\
\hline LP4-2 & 0.00 & 0.80 & 9.60 & 21.06 & 6.67 & 8.13 & 0.39 & 52.44 & 0.13 & 99.22 & 0.59 & 0.79 & 0.384 & 0.17 & 1.41 \\
\hline LP4-3 & 0.00 & 0.76 & 9.50 & 20.72 & 6.81 & 8.28 & 0.40 & 52.61 & 0.21 & 99.29 & 0.58 & 0.79 & 0.380 & 0.17 & 1.41 \\
\hline LP4-4 & 0.00 & 0.80 & 9.46 & 22.70 & 4.57 & 7.28 & 0.39 & 54.31 & 0.17 & 99.68 & 0.64 & 0.79 & 0.379 & 0.12 & 1.46 \\
\hline LP4-5 & 0.00 & 0.72 & 9.31 & 21.80 & 6.02 & 7.41 & 0.32 & 53.66 & 0.15 & 99.38 & 0.62 & 0.79 & 0.373 & 0.15 & 1.44 \\
\hline LP4-6 & 0.00 & 0.69 & 9.30 & 22.15 & 5.57 & 7.31 & 0.37 & 53.66 & 0.15 & 99.19 & 0.63 & 0.79 & 0.374 & 0.14 & 1.45 \\
\hline LP4-7 & 0.00 & 0.61 & 8.56 & 22.33 & 4.11 & 7.19 & 0.39 & 56.12 & 0.16 & 99.46 & 0.64 & 0.81 & 0.345 & 0.11 & 1.52 \\
\hline LP4-8 & 0.00 & 0.74 & 9.44 & 22.36 & 5.24 & 7.30 & 0.36 & 53.70 & 0.17 & 99.31 & 0.63 & 0.79 & 0.380 & 0.13 & 1.45 \\
\hline LP4-9 & 0.00 & 0.73 & 10.16 & 22.73 & 2.57 & 7.33 & 0.37 & 55.55 & 0.17 & 99.63 & 0.63 & 0.79 & 0.406 & 0.07 & 1.49 \\
\hline LP4-10 & 0.00 & 0.63 & 9.94 & 22.62 & 4.19 & 7.20 & 0.30 & 54.33 & 0.17 & 99.37 & 0.64 & 0.79 & 0.398 & 0.11 & 1.46 \\
\hline LP4-11 & 0.00 & 0.56 & 9.71 & 20.48 & 4.23 & 8.50 & 0.37 & 55.31 & 0.23 & 99.39 & 0.57 & 0.79 & 0.386 & 0.11 & 1.48 \\
\hline LP6-1 & 0.00 & 0.67 & 9.68 & 20.74 & 3.84 & 8.54 & 0.40 & 55.91 & 0.20 & 99.98 & 0.58 & 0.79 & 0.383 & 0.10 & 1.48 \\
\hline LP6-2 & 0.00 & 0.63 & 9.58 & 20.74 & 4.17 & 8.35 & 0.44 & 55.30 & 0.21 & 99.42 & 0.58 & 0.79 & 0.382 & 0.11 & 1.48 \\
\hline LP6-3 & 0.00 & 0.70 & 9.58 & 21.42 & 3.16 & 8.14 & 0.40 & 56.89 & 0.18 & 100.46 & 0.60 & 0.80 & 0.378 & 0.08 & 1.51 \\
\hline LP6-4 & 0.00 & 0.69 & 11.24 & 23.34 & 3.64 & 6.97 & 0.35 & 53.43 & 0.21 & 99.85 & 0.65 & 0.76 & 0.447 & 0.09 & 1.43 \\
\hline LP6-5 & 0.00 & 0.52 & 10.28 & 23.45 & 3.50 & 6.72 & 0.39 & 54.58 & 0.17 & 99.61 & 0.66 & 0.78 & 0.412 & 0.09 & 1.47 \\
\hline LP6-6 & 0.00 & 0.66 & 10.86 & 24.28 & 4.29 & 6.30 & 0.32 & 52.65 & 0.19 & 99.54 & 0.68 & 0.76 & 0.436 & 0.11 & 1.42 \\
\hline LP6-7 & 0.00 & 0.58 & 10.90 & 22.64 & 3.98 & 7.24 & 0.35 & 53.68 & 0.18 & 99.54 & 0.64 & 0.77 & 0.434 & 0.10 & 1.43 \\
\hline LP6-8 & 0.00 & 0.61 & 9.44 & 25.54 & 3.64 & 5.62 & 0.35 & 55.71 & 0.16 & 101.07 & 0.72 & 0.80 & 0.378 & 0.09 & 1.50 \\
\hline LP6-9 & 0.00 & 0.67 & 11.17 & 24.20 & 3.51 & 6.62 & 0.32 & 53.81 & 0.12 & 100.42 & $0.6 \overline{7}$ & 0.76 & 0.443 & 0.09 & 1.43 \\
\hline \multirow[t]{2}{*}{ LP-Average } & 0.00 & 0.68 & 9.87 & 22.31 & 4.52 & 7.43 & 0.37 & 54.30 & 0.18 & 99.65 & 0.63 & 0.79 & 0.39 & 0.12 & 1.46 \\
\hline & \multicolumn{15}{|c|}{ Nuasahi (chromitite fragment Breccia Zone) } \\
\hline NS9-1 & 0.02 & 0.13 & 22.35 & 25.44 & 6.64 & 6.34 & 0.33 & 38.09 & 0.21 & 99.54 & 0.69 & 0.53 & 0.854 & 0.16 & 0.98 \\
\hline NS9-10 & 0.00 & 0.13 & 22.69 & 25.32 & 6.86 & 6.53 & 0.33 & 37.95 & 0.32 & 100.13 & 0.69 & 0.53 & 0.861 & 0.17 & 0.97 \\
\hline NS9-11 ** & 0.05 & 0.32 & 25.35 & 24.70 & 6.09 & 7.09 & 0.30 & 34.80 & 0.29 & 98.99 & 0.66 & 0.48 & 0.956 & 0.15 & 0.88 \\
\hline NS9-12 ** & 0.02 & 0.25 & 25.44 & 24.63 & 6.11 & 7.11 & 0.26 & 34.98 & 0.20 & 99.00 & 0.66 & 0.48 & 0.958 & 0.15 & 0.88 \\
\hline NS9-13 ** & 0.00 & 0.22 & 22.13 & 25.47 & 7.05 & 6.34 & 0.28 & 38.03 & 0.21 & 99.73 & 0.69 & 0.54 & 0.845 & 0.17 & 0.97 \\
\hline NS9-14 ** & 0.00 & 0.18 & 25.86 & 24.43 & 6.41 & 7.34 & 0.40 & 34.99 & 0.21 & 99.82 & 0.65 & 0.48 & 0.964 & 0.15 & 0.88 \\
\hline NS9-15 ** & 0.00 & 0.25 & 21.80 & 26.68 & 5.39 & 5.60 & 0.36 & 39.77 & 0.27 & 100.13 & 0.73 & 0.55 & 0.835 & 0.13 & 1.02 \\
\hline NS9-15bis & 0.02 & 0.16 & 20.87 & 25.60 & 6.63 & 6.07 & 0.31 & 39.56 & 0.16 & 99.38 & 0.70 & 0.56 & 0.804 & 0.16 & 1.02 \\
\hline NS9-16 ** & 0.00 & 0.22 & 25.21 & 24.54 & 6.85 & 7.19 & 0.27 & 34.96 & 0.31 & 99.54 & 0.66 & 0.48 & 0.946 & 0.16 & 0.88 \\
\hline NS9-17 & 0.01 & 0.29 & 23.17 & 25.56 & 6.09 & 6.34 & 0.28 & 37.23 & 0.20 & 99.17 & 0.69 & 0.52 & 0.884 & 0.15 & 0.95 \\
\hline NS9-19** & 0.01 & 0.04 & 23.17 & 25.70 & 5.23 & 6.31 & 0.37 & 39.38 & 0.16 & 100.35 & 0.70 & 0.53 & 0.875 & 0.13 & 1.00 \\
\hline NS9-20 ** & 0.03 & 0.49 & 19.86 & 26.47 & 6.71 & 5.85 & 0.28 & 40.67 & 0.20 & 100.55 & 0.72 & 0.58 & 0.762 & 0.16 & 1.05 \\
\hline NS9-21 & 0.00 & 0.13 & 22.84 & 25.61 & 6.37 & 6.36 & 0.27 & 38.28 & 0.22 & 100.08 & 0.69 & 0.53 & 0.866 & 0.15 & 0.97 \\
\hline NS9-26 & 0.00 & 0.15 & 23.04 & 25.28 & 6.37 & 6.40 & 0.31 & 37.39 & 0.23 & 99.18 & 0.69 & 0.52 & 0.880 & 0.16 & 0.96 \\
\hline NS9-29 & 0.00 & 0.23 & 21.28 & 26.48 & 6.23 & 5.72 & 0.38 & 39.88 & 0.29 & 100.49 & 0.72 & 0.56 & 0.814 & 0.15 & 1.02 \\
\hline NS9-4** & 0.00 & 0.71 & 20.53 & 25.86 & 6.13 & 6.24 & 0.33 & 39.82 & 0.29 & 99.91 & 0.70 & 0.57 & 0.789 & 0.15 & 1.03 \\
\hline NS9-6 ** & 0.00 & 0.24 & 22.95 & 25.15 & 6.73 & 6.60 & 0.31 & 37.32 & 0.26 & 99.56 & 0.68 & 0.52 & 0.873 & 0.16 & 0.95 \\
\hline NS9-7 ** & 0.05 & 0.21 & 21.12 & 26.37 & 7.05 & 5.73 & 0.46 & 39.10 & 0.27 & 100.36 & 0.72 & 0.55 & 0.809 & 0.17 & 1.00 \\
\hline NS9-9 ** & 0.04 & 0.26 & 22.84 & 26.04 & 6.55 & 6.22 & 0.37 & 37.84 & 0.22 & 100.37 & 0.70 & 0.53 & 0.865 & 0.16 & 0.96 \\
\hline NS9-9bis & 0.01 & 0.26 & 22.40 & 25.72 & 6.38 & 6.28 & 0.32 & 38.34 & 0.24 & 99.94 & 0.70 & 0.53 & 0.853 & 0.16 & 0.98 \\
\hline \multirow[t]{2}{*}{ NS-Average } & 0.01 & 0.24 & 22.74 & 25.55 & 6.39 & 6.38 & 0.33 & 37.92 & 0.24 & 99.81 & 0.69 & 0.53 & 0.86 & 0.16 & 0.97 \\
\hline & \multicolumn{15}{|c|}{ Cedrolina chromitite } \\
\hline CD100-1 ** & 0.00 & 0.25 & 8.10 & 29.83 & 9.70 & 1.89 & 0.78 & 49.51 & 0.08 & 100.14 & 0.90 & 0.80 & 0.339 & 0.26 & 1.39 \\
\hline CD100-2 ** & 0.00 & 0.41 & 6.66 & 30.47 & 11.03 & 1.58 & 0.76 & 49.89 & 0.12 & 100.92 & 0.92 & 0.83 & 0.279 & 0.30 & 1.40 \\
\hline CD100-3 ** & 0.00 & 0.15 & 5.78 & 30.17 & 12.45 & 1.48 & 0.92 & 50.20 & 0.11 & 101.26 & 0.92 & 0.85 & 0.243 & 0.33 & 1.41 \\
\hline CD100-4 ** & 0.00 & 0.30 & 7.79 & 30.28 & 10.83 & 1.75 & 0.77 & 49.15 & 0.15 & 101.02 & 0.91 & 0.81 & 0.324 & 0.29 & 1.37 \\
\hline CD105A-5 ** & 0.01 & 1.10 & 10.16 & 28.29 & 0.68 & 3.95 & 0.52 & 55.56 & 0.14 & 100.40 & 0.80 & 0.79 & 0.412 & 0.02 & 1.51 \\
\hline CD105C-2 ** & 0.02 & 0.17 & 9.15 & 24.33 & 2.51 & 5.82 & 0.51 & 57.56 & 0.14 & 100.24 & 0.70 & 0.81 & 0.369 & 0.06 & 1.56 \\
\hline CD105C-1 ** & 0.09 & 0.03 & 5.81 & 25.12 & 2.50 & 4.74 & 0.67 & 60.75 & 0.14 & 99.84 & 0.75 & 0.88 & 0.240 & 0.07 & 1.69 \\
\hline CD105C-3 ** & 0.09 & 0.06 & 4.08 & 25.74 & 1.99 & 4.31 & 0.66 & 63.45 & 0.21 & 100.58 & 0.77 & 0.91 & 0.169 & 0.05 & 1.77 \\
\hline CD105C-4 ** & 0.08 & 0.15 & 11.10 & 24.53 & 2.02 & 5.95 & 0.55 & 55.79 & 0.16 & 100.32 & 0.70 & 0.77 & 0.443 & 0.05 & 1.49 \\
\hline CD106D-1 ** & 0.03 & 0.06 & 5.98 & 29.22 & 6.65 & 1.98 & 1.05 & 55.76 & 0.19 & 100.91 & 0.89 & 0.86 & 0.250 & 0.18 & 1.57 \\
\hline CD106D-2 ** & 0.00 & 0.08 & 7.89 & 28.93 & 6.79 & 2.21 & 1.14 & 53.30 & 0.13 & 100.47 & 0.88 & 0.82 & 0.328 & 0.18 & 1.49 \\
\hline CD106D-3 ** & 0.01 & 0.60 & 8.39 & 29.23 & 6.10 & 2.38 & 1.00 & 52.17 & 0.17 & 100.04 & 0.87 & 0.81 & 0.349 & 0.16 & 1.46 \\
\hline CD106D-4 ** & 0.03 & 0.17 & 10.01 & 29.20 & 7.13 & 2.47 & 0.98 & 50.51 & 0.12 & 100.61 & 0.87 & 0.77 & 0.411 & 0.19 & 1.39 \\
\hline CD47A-1 ** & 0.03 & 0.05 & 7.21 & 25.32 & 2.91 & 4.81 & 0.77 & 59.33 & 0.18 & 100.61 & 0.75 & 0.85 & 0.294 & 0.08 & 1.62 \\
\hline CD47A-4 ** & 0.00 & 0.08 & 10.13 & 25.28 & 3.94 & 5.22 & 0.60 & 55.11 & 0.19 & 100.55 & 0.73 & 0.78 & 0.407 & 0.10 & 1.49 \\
\hline CD $47 A-3 * *$ & 0.00 & 0.09 & 9.57 & 25.44 & 4.19 & 5.02 & 0.71 & 55.49 & 0.19 & 100.69 & 0.74 & 0.80 & 0.386 & 0.11 & 1.50 \\
\hline CD47A-2 ** & 0.03 & 0.07 & 7.91 & 25.42 & 3.81 & 4.82 & 0.76 & 57.65 & 0.13 & 100.59 & 0.75 & 0.83 & 0.322 & 0.10 & 1.57 \\
\hline CD47B-1 ** & 0.00 & 0.01 & 5.69 & 24.38 & 4.41 & 5.31 & 0.64 & 60.09 & 0.17 & 100.72 & 0.72 & 0.88 & 0.233 & 0.12 & 1.65 \\
\hline CD47C-1 ${ }^{* *}$ & 0.00 & 0.74 & 9.80 & 20.14 & 8.60 & 8.71 & 0.63 & 51.08 & 0.18 & 99.86 & 0.56 & 0.78 & 0.388 & 0.22 & 1.36 \\
\hline CD12an16 & 0.00 & 0.31 & 9.54 & 22.86 & 4.24 & 6.62 & 0.66 & 55.13 & 0.18 & 99.54 & 0.66 & 0.79 & 0.384 & 0.11 & 1.49 \\
\hline CD-Average & 0.02 & 0.24 & 8.04 & 26.71 & 5.62 & 4.05 & 0.75 & 54.87 & 0.15 & 100.47 & 0.79 & 0.82 & 0.33 & 0.15 & 1.51 \\
\hline
\end{tabular}

$\mathrm{Fe}_{2} \mathrm{O}_{3}=$ calculated assuming the ideal spinel stoichiometry. $\mathrm{Fe}^{2} \#=\mathrm{Fe}^{2} /\left(\mathrm{Fe}^{2}+\mathrm{Mg}\right) . \mathrm{Cr} \#=\mathrm{Cr} /(\mathrm{Cr}+\mathrm{Al}) . \mathrm{Al}, \mathrm{Fe}{ }^{3}, \mathrm{Cr}$ in at $\%$. ${ }^{*}=$ analyses published with label CED by [13]. 

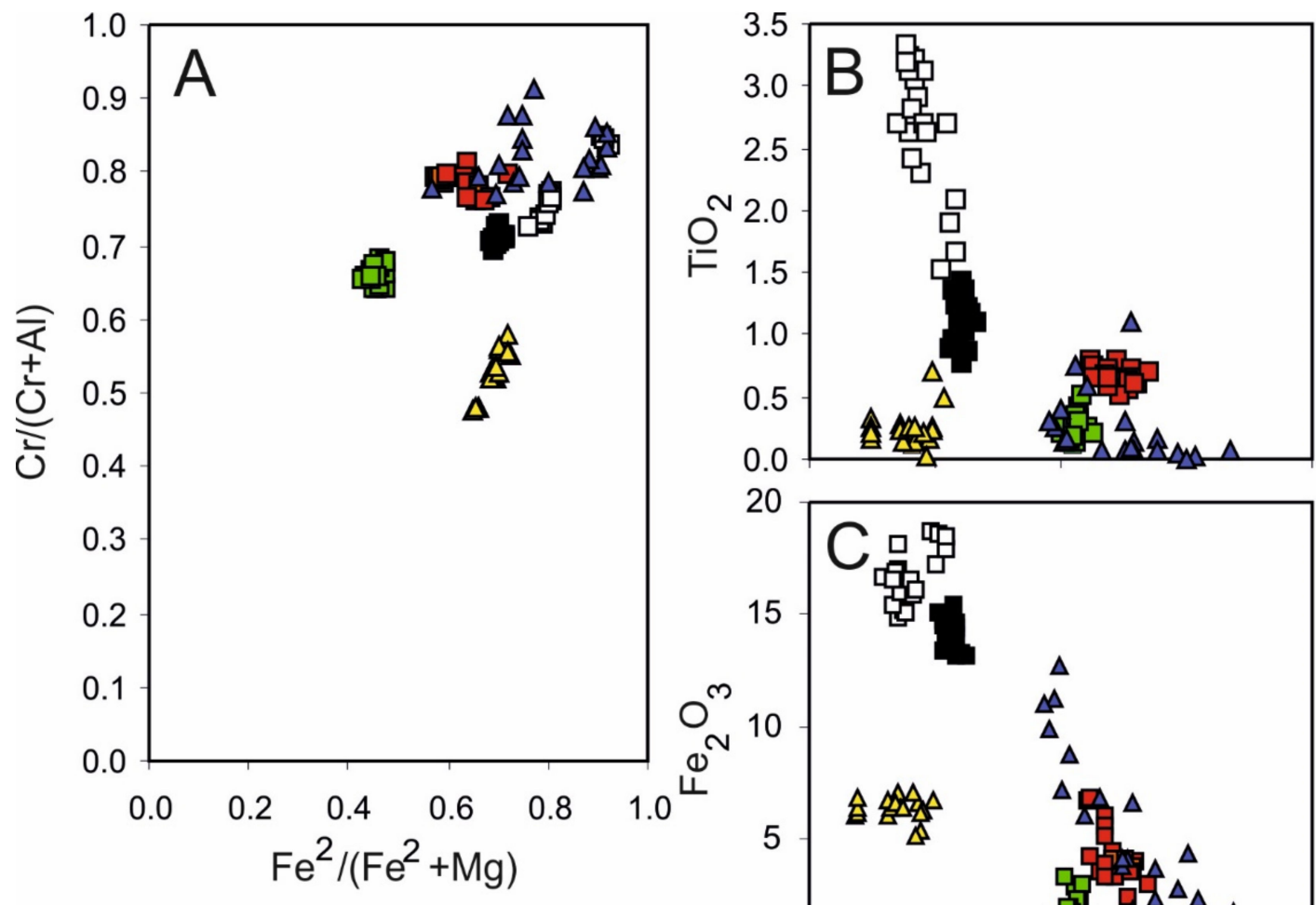

Magmatic chromitites:

- UG $=$ UG2

$\square \mathrm{MR}=$ Merensky Reef

$\square \mathrm{JC}=$ Jacurici

- LP = Loma Peguera

Metamorphic chromitites:

$\triangle \mathrm{NS}=$ Nuasahi

$\triangle \mathrm{CD}=$ Cedrolina
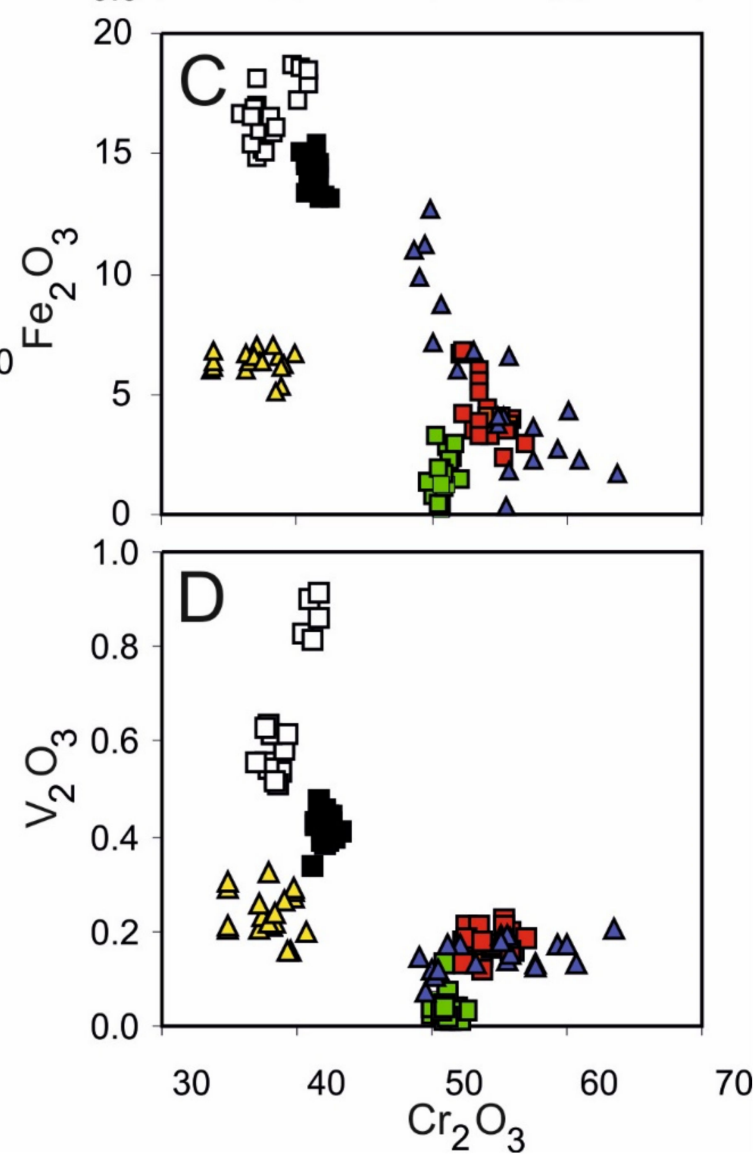

Figure 3. Variation of chromite composition in the chromitite samples investigated in this work. Chromium number $\mathrm{Cr} /(\mathrm{Cr}+\mathrm{Al})$ versus bivalent iron number $\mathrm{Fe}^{2} /\left(\mathrm{Fe}^{2}+\mathrm{Mg}\right)$ (concentration as atoms \%) (A). Correlation between $\mathrm{TiO}_{2}, \mathrm{Fe}_{2} \mathrm{O}_{3}$ and $\mathrm{V}_{2} \mathrm{O}_{3}$ as function of $\mathrm{Cr}_{2} \mathrm{O}_{3}$ (concentrations as oxide weight \%) (B-D).

\subsection{Unmetamorphosed (Magmatic) Chromitite}

Based on the concept that the chromite mineral chemistry is strictly related to the composition of the parental magmas ([53], and references therein) compositional variations observed in the chromitites devoid of high-grade metamorphism can be interpreted as a reflection of magmatic processes leading to precipitation of massive chromitite. Paleoproterozoic-Archaean layered intrusions of the world formed by mixing and fractional crystallization of variable proportions of high-Mg, basaltic andesite (U-type magma) with tholeiite-type mafic magmas [27], that may drive the rhythmical stabilization of chromitite during fractionation [54].

The UG2 and MR chromitites crystallized from relatively evolved parent melts, in the highest stratigraphic levels of the Critical Zone. They have high concentrations of $\mathrm{TiO}_{2}, \mathrm{Fe}_{2} \mathrm{O}_{3}$, and $\mathrm{V}_{2} \mathrm{O}_{3}$, (Figure 3B-D). The high oxidation state of the MR chromite 
$\left(\mathrm{Fe}_{2} \mathrm{O}_{3}>15 \mathrm{wt} \%\right)$ and the abundance of hydrous mafic silicates in the pyroxenite host suggest crystallization under high fluid activity and oxygen fugacity [28-30,55].

The oversized chromitite layers of the Jacurici ultramafic sill require deposition from an enormous volume of magma that is not consistent with the actual size of the intrusive body [37]. According to these authors, the sill acted as a conduit through which large amounts of a primitive, high-Mg magma flowed upwards to the crust, for a long time, and formed a thick zone of chromitite by close events of chromite precipitation. The low concentration of $\mathrm{TiO}_{2}, \mathrm{~V}_{2} \mathrm{O}_{3}$, and $\mathrm{Fe}_{2} \mathrm{O}_{3}$ in the chromite (Figure 3B-D) reflects poor differentiation degree similar to magmas parental to low-Ti chromitites from other Proterozoic layered intrusions (e.g., Campo Formoso [56]). Cr saturation in the mafic magma was enhanced, if not triggered, by changes in magma composition associated with the crustal contamination event in the parent melt of the intrusion [37].

Precipitation of massive chromitite in the Mesozoic mantle-plume of Loma Caribe was a result of reaction between harzburgitic mantle and a percolating mafic magma, in the same way as podiform chromitite in upper mantle tectonite of ophiolites ([57], and references therein). The unusual geodynamic setting of this mantle fragment [39]) is responsible for the peculiar composition of the chromite ore. The high-Cr nature of the chromite (Cr\# $=0.76-0.82)$ would be consistent with crystallization from "boninite", commonly invoked as the parent melt of high-Cr podiform chromitite from supra-subduction-zone (SSZ). However, the high $\mathrm{Fe}^{2} \#=0.63$ and high titanium $\left(\mathrm{TiO}_{2}=0.68 \mathrm{wt} \%\right)$ and vanadium $\left(\mathrm{V}_{2} \mathrm{O}_{3}=0.18 \mathrm{wt} \%\right)$ (Figure $\left.3 \mathrm{~A}, \mathrm{~B}, \mathrm{D}\right)$ indicate a more evolved parent melt compared with boninite, which cannot be readily reconciled with a SSZ geodynamic setting [39,41,42].

\subsection{Metamorphic Chromitite}

The Nuasahi chromite deposit originally formed by magmatic accumulation of chromite, as usual for stratiform chromitite in layered intrusions, however, the high-grade metamorphism and recrystallization that have obliterated the igneous cumulus texture induced changes in the primary magmatic composition of chromite [23]. The low Cr\# $=0.48-0.58$ may be a magmatic relic consistent with the stratiform affinity of the chromite. Nevertheless, the high $\mathrm{Fe}^{2} \#=0.65-0.72$ relative to low $\mathrm{Cr} \#$ (Figure 3A) is a result of secondary $\mathrm{Fe}^{2}$ enrichment and/or loss of $\mathrm{Mg}$ during metamorphism [23], as expected for chrome spinels from high-grade metamorphic rocks [53,58]. Furthermore, a distinctive depletion in titanium, from 0.71 to $0.04 \mathrm{wt} \% \mathrm{TiO}_{2}$ (Figure 3B), has shifted the NS compositions out of the ideal $\mathrm{TiO}_{2}$ versus $\mathrm{Cr}_{2} \mathrm{O}_{3}$ trend formed by the unmetamorphosed stratiform chromitites of JC, UG2, and MR. We suggest that this depletion was caused by significant remobilization of Ti from the NS chromite during metamorphic recrystallization at high temperature, which likely led to subsequent rutile crystallization.

The samples from Cedrolina show the most scattered patterns in chromite composition despite of the extremely small size of the deposit [13]. The chromite composition has the highest chromium and iron numbers $\left(\mathrm{Cr} \#=0.77-0.91, \mathrm{Fe}^{2} \#=0.56-0.85\right)$ due to depletion in $\mathrm{MgO}$ and $\mathrm{Al}_{2} \mathrm{O}_{3}$, while the oxidation state increases with decreasing $\mathrm{Cr}_{2} \mathrm{O}_{3}$ in the range of $\mathrm{Fe}_{2} \mathrm{O}_{3}=0.68-12.45 \mathrm{wt} \%$ (Figure 3A,B). These wide changes in chromite composition cannot be reconciled with magmatic differentiation processes in a small batch of magma. According to [13], they are consistent with processes leading to the formation of "ferrian-chromite" under polycyclic regional metamorphism, hydrothermal metasomatism, and final supergene alteration, as already observed in other chromite deposits of Brazil $[17,37,56,59]$. In particular, an increasing oxidation state (i.e., $\mathrm{Fe}_{2} \mathrm{O}_{3}$ ) coupled with $\mathrm{Cr}_{2} \mathrm{O}_{3}$ decrease is consistent with chromium oxidation to $\mathrm{Cr}^{6+}$ and mobilization during soil formation.

\section{Rutile Modal Abundance and Textural Relations}

Despite small grain sizes (sometimes $<10 \mu \mathrm{m}$ ), rutile was easily recognized under the reflected-light microscope because of its yellow-orange plane-polarized internal reflection (Figure 4A,B). On this basis, simple point-counting analysis yielded modal abundances 
between $0.5 \%$ and $2 \%$ by volume, increasing from the magmatic chromitites (in the order $\mathrm{LP}, \mathrm{JC}, \mathrm{UG} 2, \mathrm{MR})$ to the metamorphic ones (CD, NS).
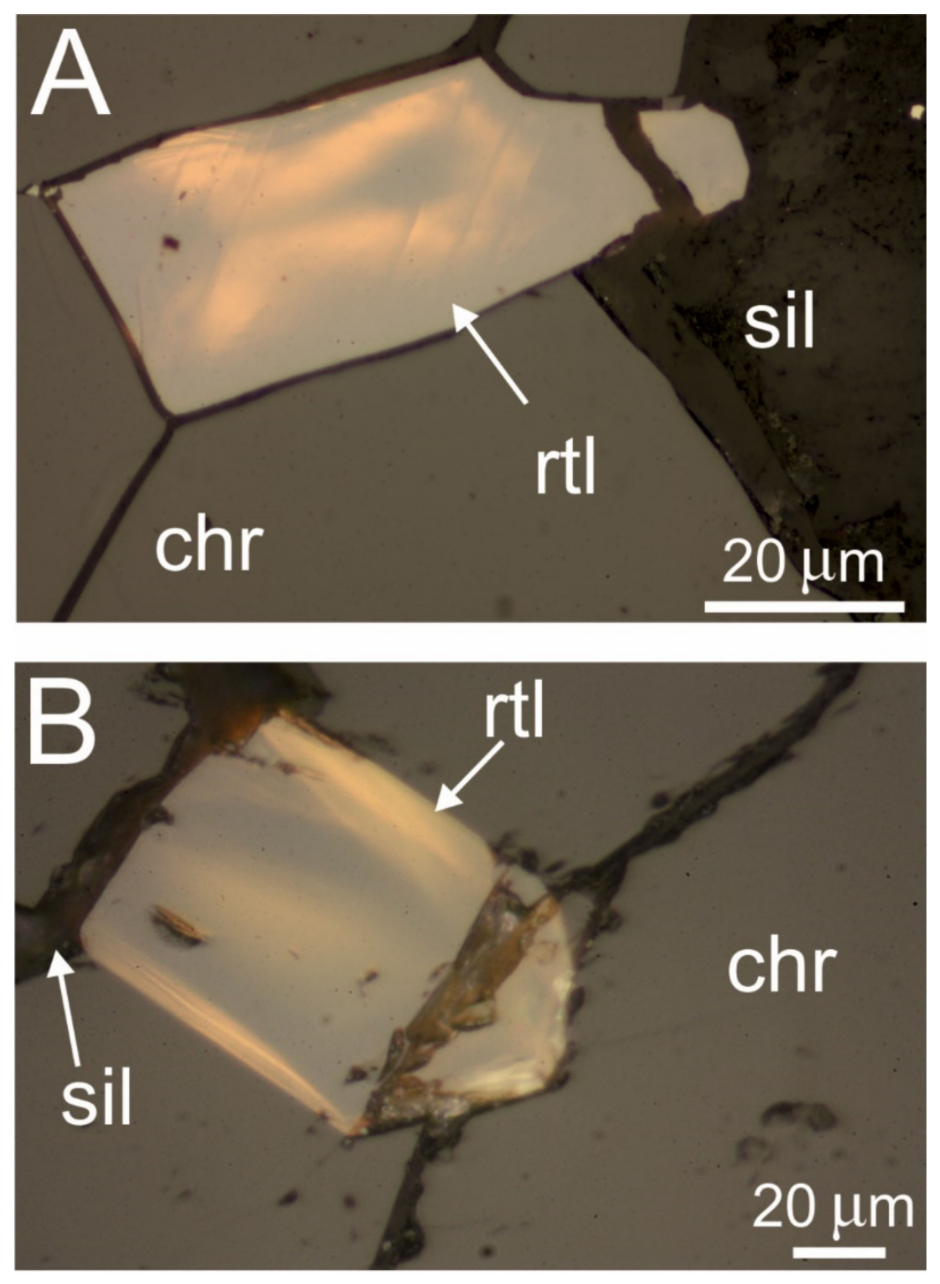

Figure 4. Digital image in reflected plane polarized light showing internal reflection in rutile crystals from the Jacurici (A) and UG2 (B) chromitite. Abbreviations: rtl, rutile; others as in Figure 1.

Similarly, the maximum grain size of rutile roughly follows the same trend, increasing from below $20 \mu \mathrm{m}$ in LP chromitite up to more than $200 \mu \mathrm{m}$ in those of NS. Consistently, morphology and textural relations with the host chromite vary remarkably from magmatic chromitites (UG2, MR, JC, LP) to the metamorphic ones (CD, NS), and show a variegated typology of cases much more complex compared with rutile described from mafic and ultramafic rocks of the Bushveld complex ([14], and references therein).

In cumulus chromitites of UG2, MR, and JC, rutile has a very similar mode of occurrence. Most commonly, it occurs as irregular patches typically molded onto chromite grains accumulated by gravity settling (Figure $5 \mathrm{~A}$ ), or as euhedral to sub-euhedral grains interstitial to chromite (Figure 5B,C), resembling an intercumulus phase in both cases. At Merensky Reef, rutile forms anhedral grains overgrowing chromite at the contact with clinopyroxene of the silicate matrix (Figures $5 \mathrm{E}$ and $1 \mathrm{~B}$ boxes $1,2,3$ ) or occurs in silicate pockets (orthopyroxene, amphibole replaced by chlorite) occluded among chromite grains, although maintaining contact with chromite (Figure 5D). Another interesting association in the Merensky Reef consists of rutile flame-like lamellae located inside ilmenite interstitial to chromite and large sulfide aggregates (Figure 5F). Ilmenite appears to have replaced rutile and includes a droplet of $\mathrm{Pt}-\mathrm{Fe}$ and Ni-sulfide, while amphibole and chlorite mark the contact ilmenite-sulfide. 

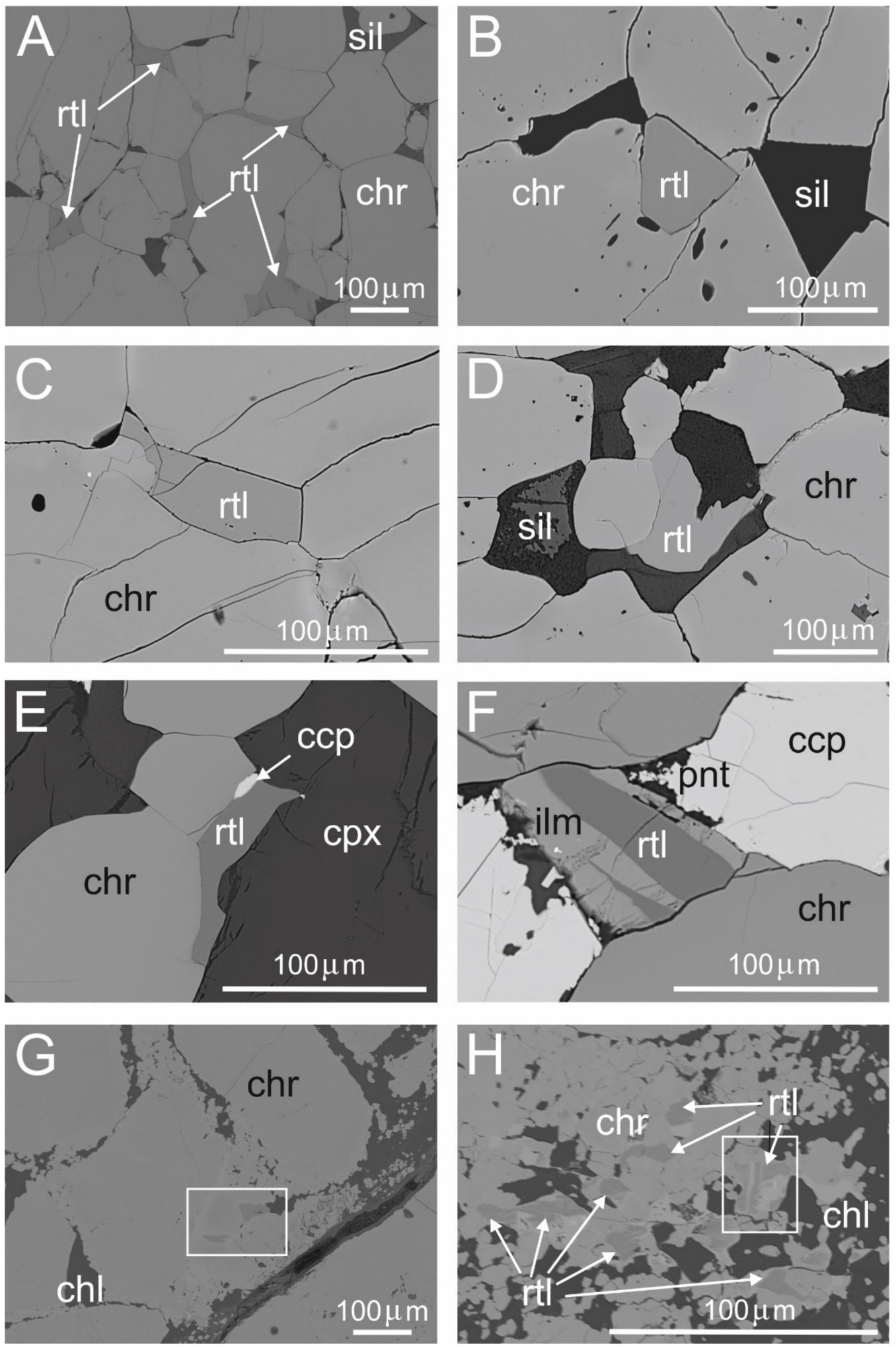

Figure 5. Texture typology of rutile associated with magmatic chromite deposits (BSE images). Net-textured rutile molded on cumulus chromite grains (UG2 chromitite) (A). Anhedral rutile interstitial to cumulus chromite (Jacurici samples JC135 and JC126) (B,C). Anhedral rutile locked in a silicate pocket interstitial to chromite (Jacurici sample JC135) (D). Rutile overgrowing chromite at the contact with clinopyroxene (Merensky Reef) (E). Rutile flames in ilmenite interstitial to chromite and sulfide aggregates (Merensky Reef) (F). Rutile in partially altered massive chromite (Loma Peguera, sample LP4) (G). Rutile and chromite, both replaced by ilmenite (Loma Peguera, sample LP4) (H). Boxes in G and H indicate position of rutile grains. Abbreviations: ccp, chalcopyrite; pnt, pentlandite; ilm, ilmenite; others as in Figure 1. 
Rutile in the LP chromitite may occur as irregular grains included in partially altered massive chromite, sometimes in contact with altered silicates (Figure 5G). Complex assemblages of chromite-rutile, both replaced by ilmenite, occur in shear zones dominated by interlocking fine crystals of chromite and altered silicates forming a "mortar-like" texture (Figure 5H).

In the metamorphic chromitite of Cedrolina, [13] described relatively large rutile grains (usually $>10 \mu \mathrm{m}$ ) in variable textural relations with altered chromite. Rutile may occur inside fractured chromite nodules or adjacent to grain boundaries, typically in contact with the chlorite-talc matrix (Figure 6A-D). Notably, some rutile grains include fragments of chromite, possibly indicating crystallization during or soon after chromite deformation (Figure 6B,D). Rutile crystals of various sizes also occur in the chlorite-talc matrix around chromite nodules or filling crosscutting fractures. These rutiles are frequently found in association with zircon (Figure 6E,F), or appear to have been replaced by ilmenite with proceeding hydrothermal metasomatism (Figure 6G,H). The rutile in the metamorphic chromitite fragment of the Nuasahi Breccia Zone essentially consists of large (up to $>200 \mu \mathrm{m}$ ) anhedral grains included in altered chromite (Figure 7A), filling chromite fractures (Figure 7B), or located at the chromite-silicate contact (Figure 7C,D). The typically irregular morphology suggests coarse-grain recrystallization and further alteration of the chromite and silicate matrix.
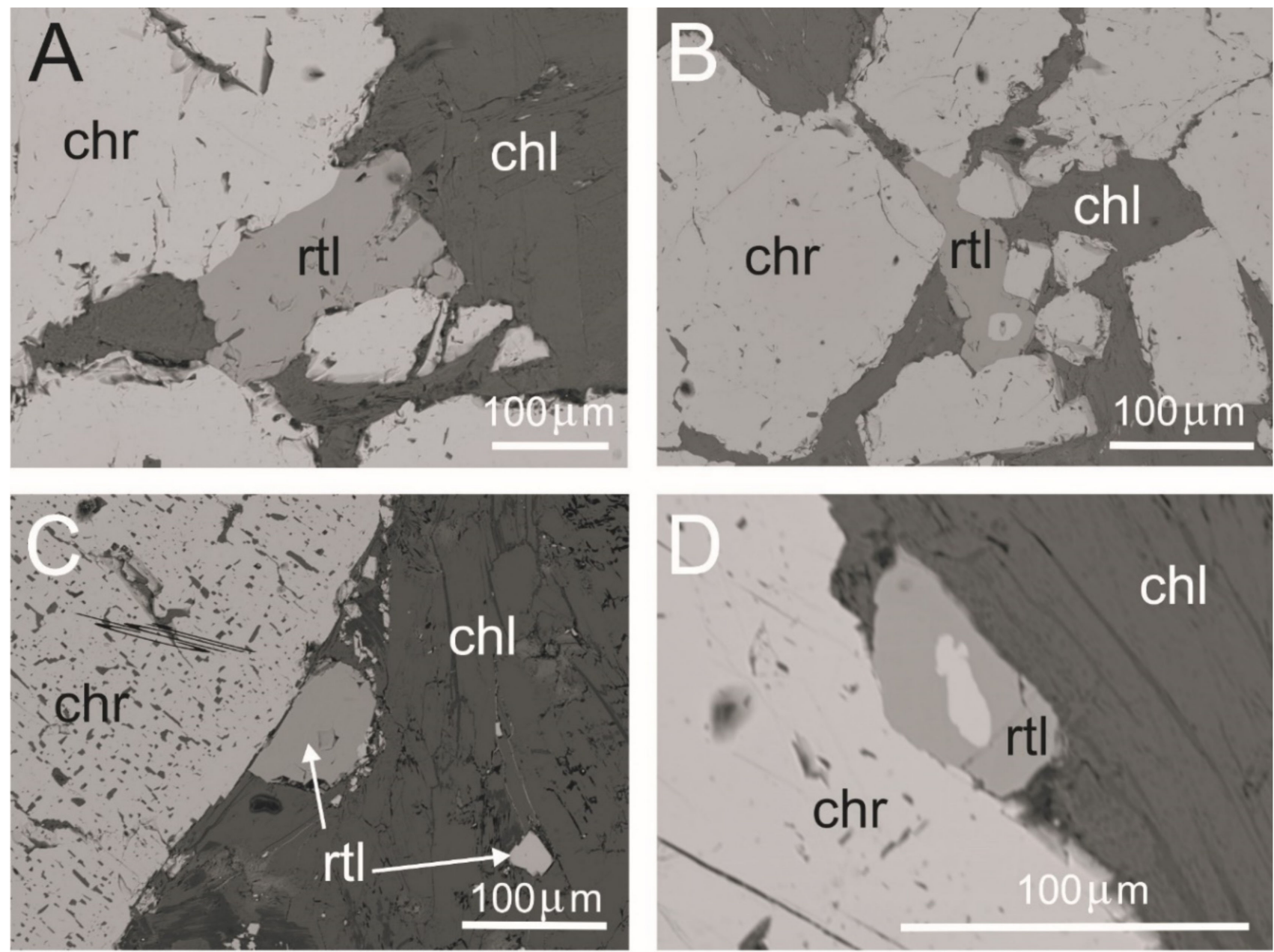

Figure 6. Cont. 

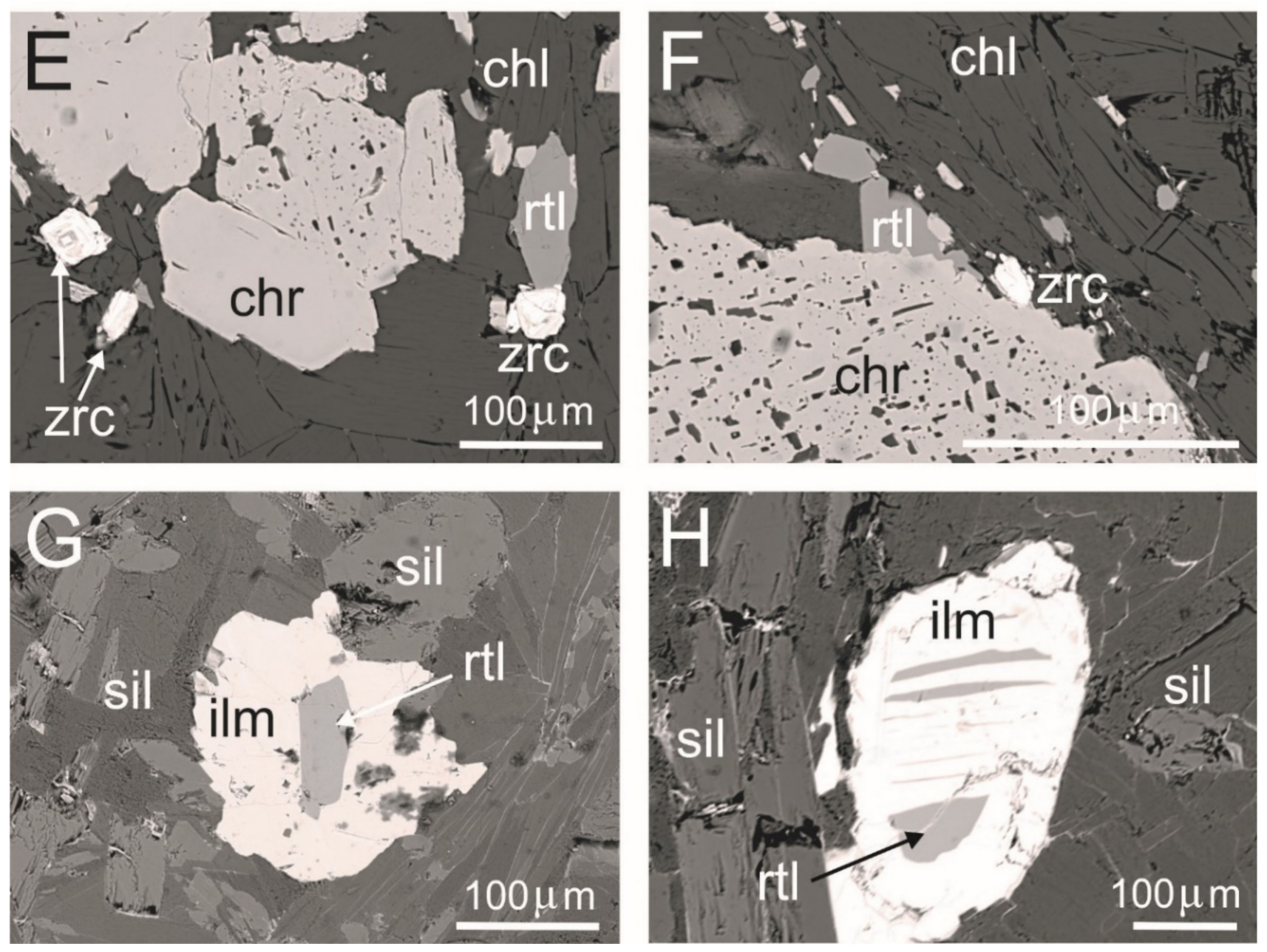

Figure 6. Texture typology of rutile in the metamorphic chromitite of Cedrolina (BSE images). Large rutile grains interstitial between chromite and chlorite (sample CD100) (A,B). Rutile grains attached to the boundary of altered chromite (samples CD105B and CD106D) (C,D). Notably, rutile in Figure 6B,D contains chromite inclusions. Rutile-zircon association in chlorite and attached to altered chromite (sample CD105C) (E,F). Rutile replaced by ilmenite in the altered matrix (sample CD169) (G,H). Abbreviations: zrc, zircon; others as in Figures 1 and 5.
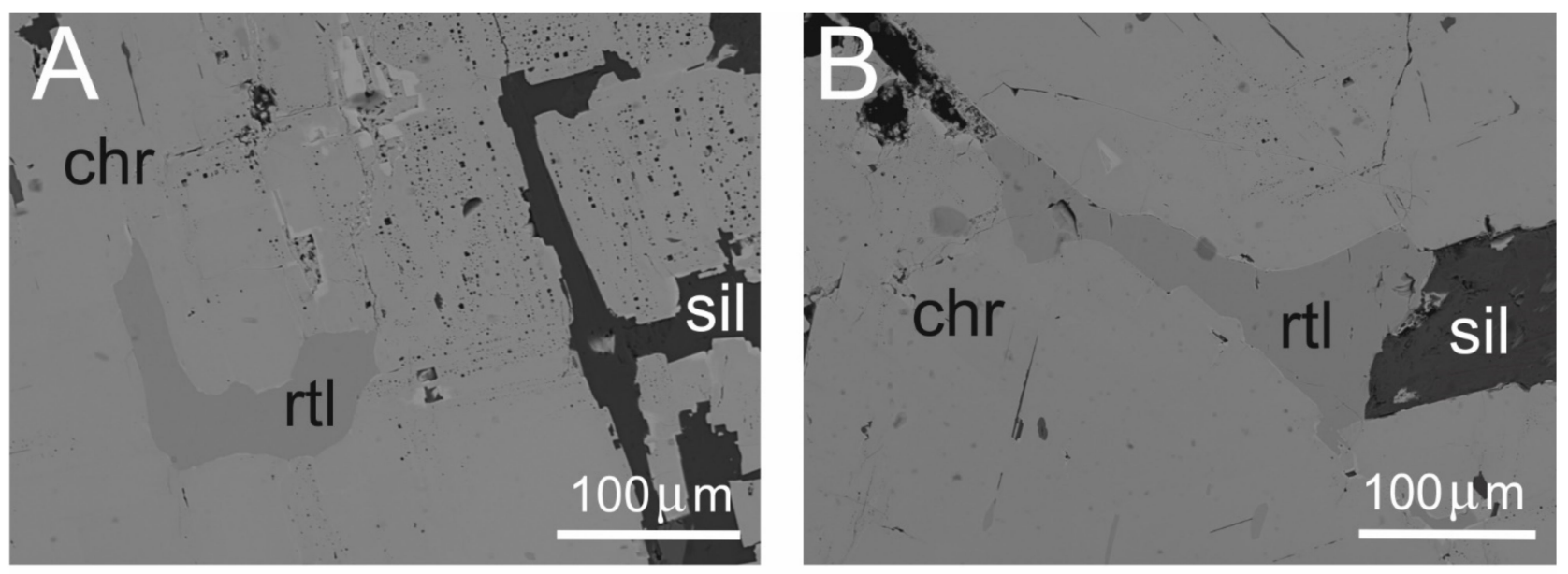

Figure 7. Cont. 

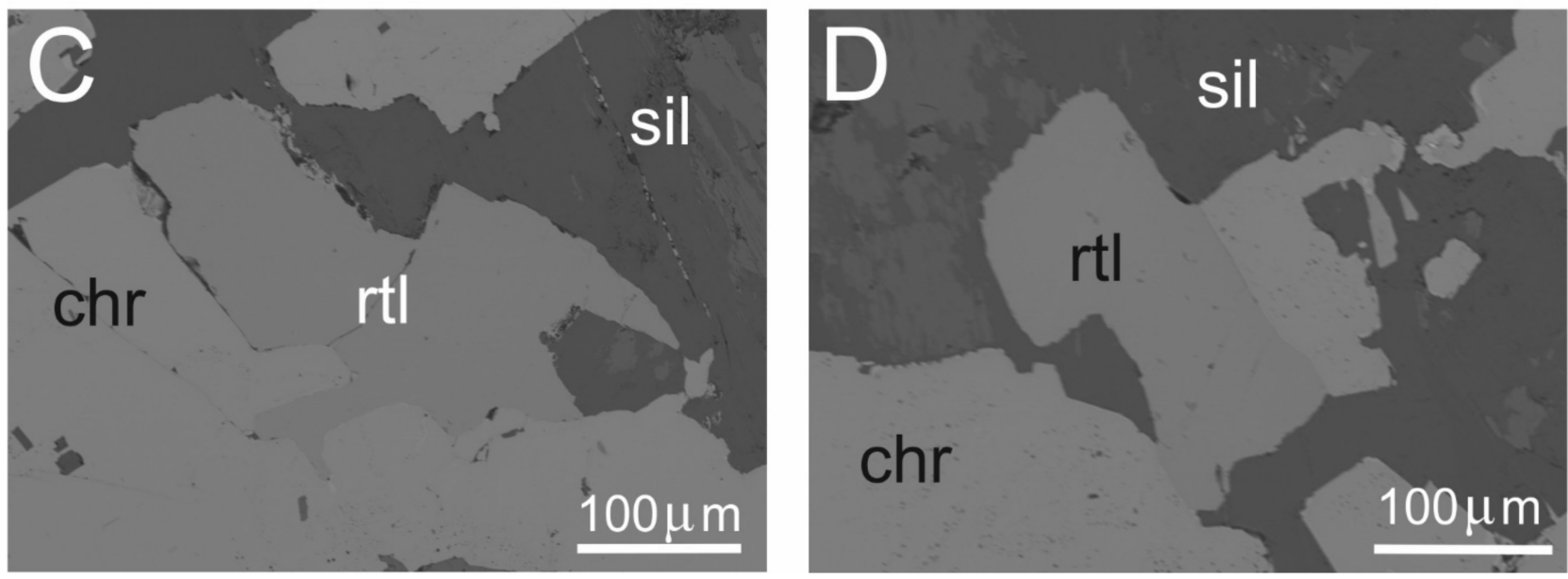

Figure 7. Texture typology of rutile associated with the metamorphic chromitite of Nuasahi (BSE images). Large, anhedral rutile grains associated with recrystallized and altered chromite (sample NS9) (A,B). Note the acicular rutile inclusions in chromite (B). Large, anhedral rutile grains at the boundary chromite-silicate (sample NS9) (C,D). Abbreviations as in Figures 1,4 and 6.

All of the studied types of chromitite were found to host rutile as minute inclusions that rarely exceed $10 \mu \mathrm{m}$ in size. In the magmatic chromitites, rutile commonly occurs as isolated polygonal crystals engulfed in unaltered solid chromite, away from cracks and fissures (Figure 8A). The largest rutile inclusions (up to $\sim 50 \mu \mathrm{m}$ ) were observed in the Nuasahi sample, characterized by rounded or irregular morphology, and frequently associated with fissures in the altered domains of chromite (Figure 8B-D). At Cedrolina, minute rutile grains $(<10 \mu \mathrm{m})$ along with chlorite fill the holes in riddled chromite (Figure $8 \mathrm{~F}$ ). Furthermore, idiomorphic rutile with a tiny droplet of uraninite occurs in the core of a large chromite grain with increasing alteration at the rim (Figure 8G,H). Conspicuously, tiny rutile grains with irregular shape are visible in the chlorite-talc matrix (Figure 8G, left and upper corner), indicating two different stages of secondary rutile crystallization. A peculiar type of rutile inclusion was observed in metamorphic chromitites (NS, CD), characterized by swarms of acicular rutile needles developed along cubic cleavage planes of highly altered chromite (Figure 8E).
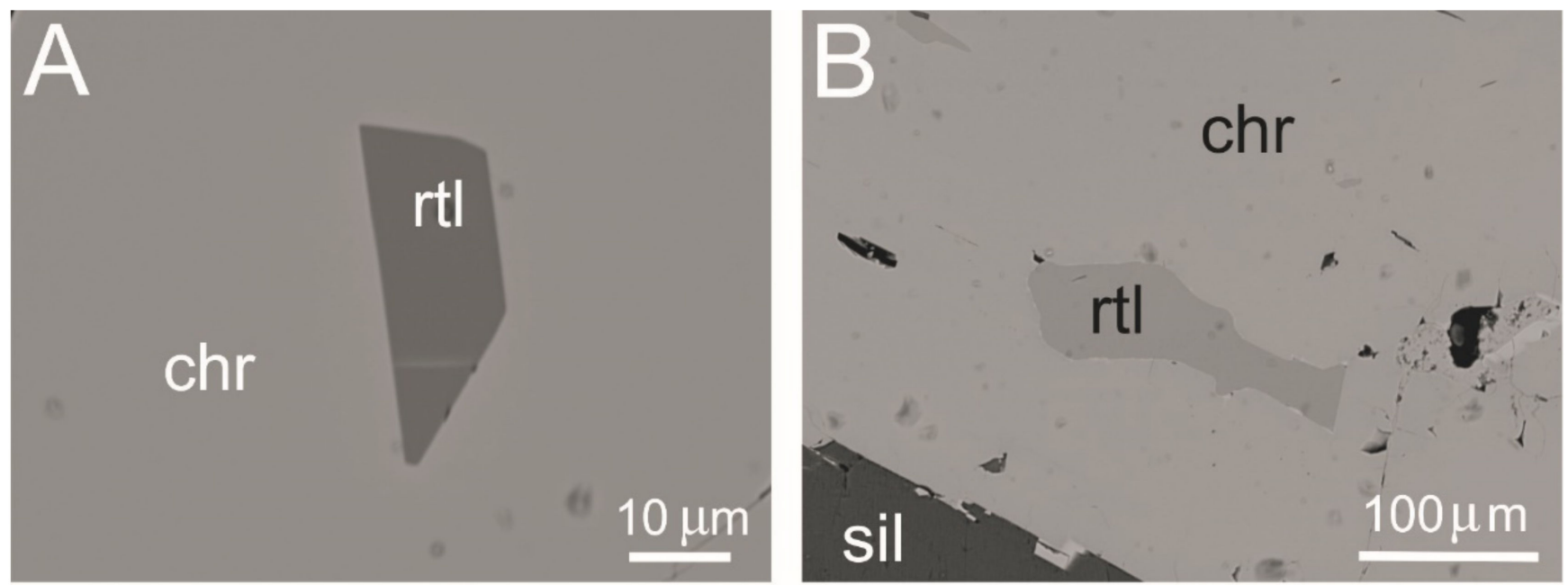

Figure 8. Cont. 

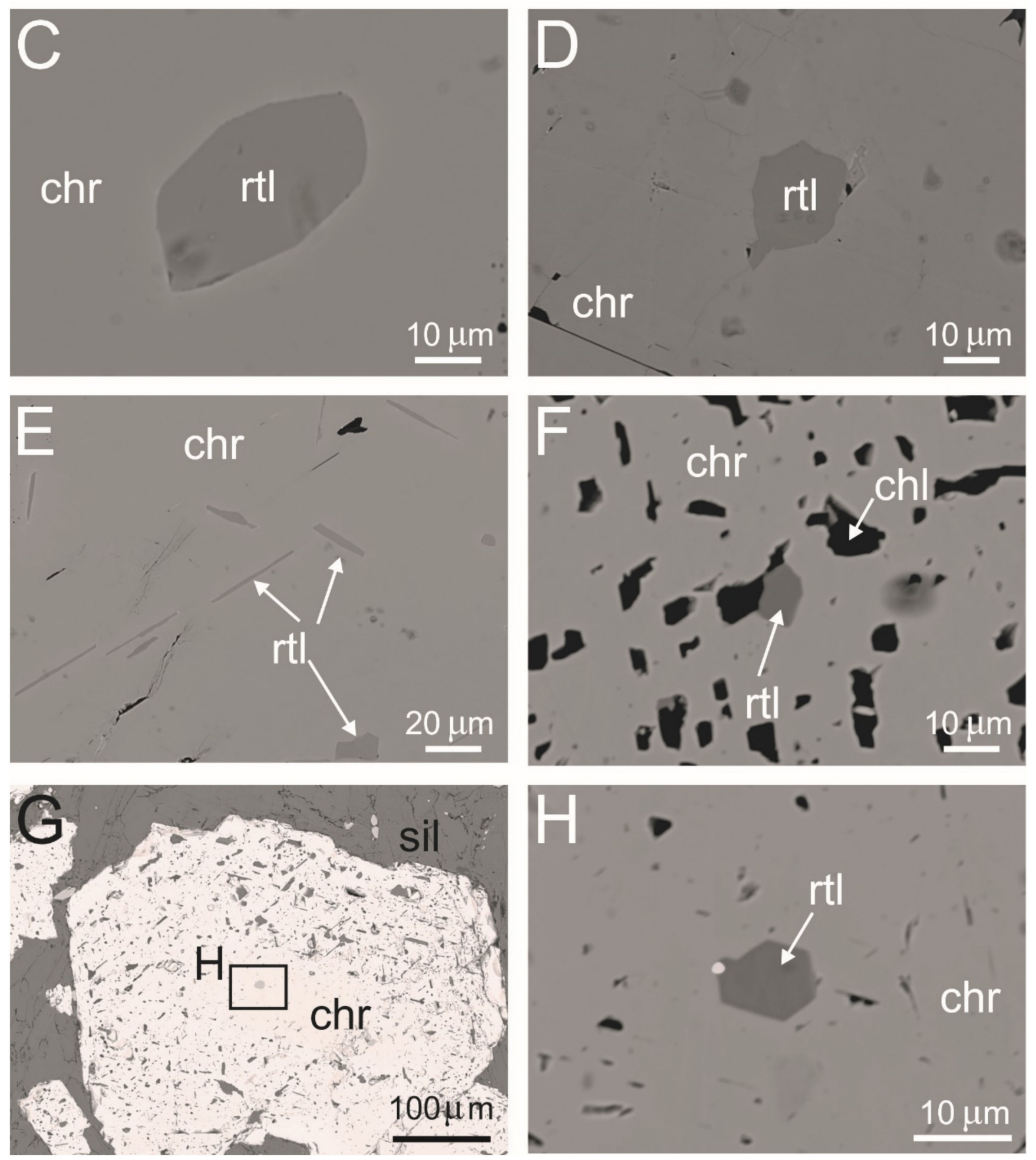

Figure 8. BSE images of rutile inclusions in chromite from various localities. Polygonal rutile included in chromite of Merensky Reef (A). Anhedral rutile included in altered chromite of Nuasahi (sample NS9) (B-D). Tiny acicular needles of rutile developed according to cubic symmetry of recrystallized chromite of Nuasahi (sample NS9) (E). Small rutile crystals included in chromite riddled with holes (Cedrolina, sample CD105C) (F). Euhedral rutile with a tiny droplet of uraninite (white spot) in the core of altered chromite grain (Cedrolina, sample CD47C) $(\mathbf{G}, \mathbf{H})$. Abbreviations as in Figures 1 and 4.

At all localities, except Cedrolina, sub-euhedral rutile occurs as composite aggregates with PGM, Ni-Cu sulfides, and silicate, completely embedded in chromite. 
The present examples reported from Loma Peguera (Figure 9A) and Nuasahi (Figure 9B,C) confirm previous studies [23,60,61], which documented primary magmatic inclusions of rutile plus laurite, Os-Ir alloy, Ru-rich pentlandite (Bushveld), rutile plus laurite-irarsite-pyrrhotite or laurite-cuproiridsite (Loma Peguera), and rutile plus lauriteirarsite-pentlandite, or laurite-silicate (Nuasahi).
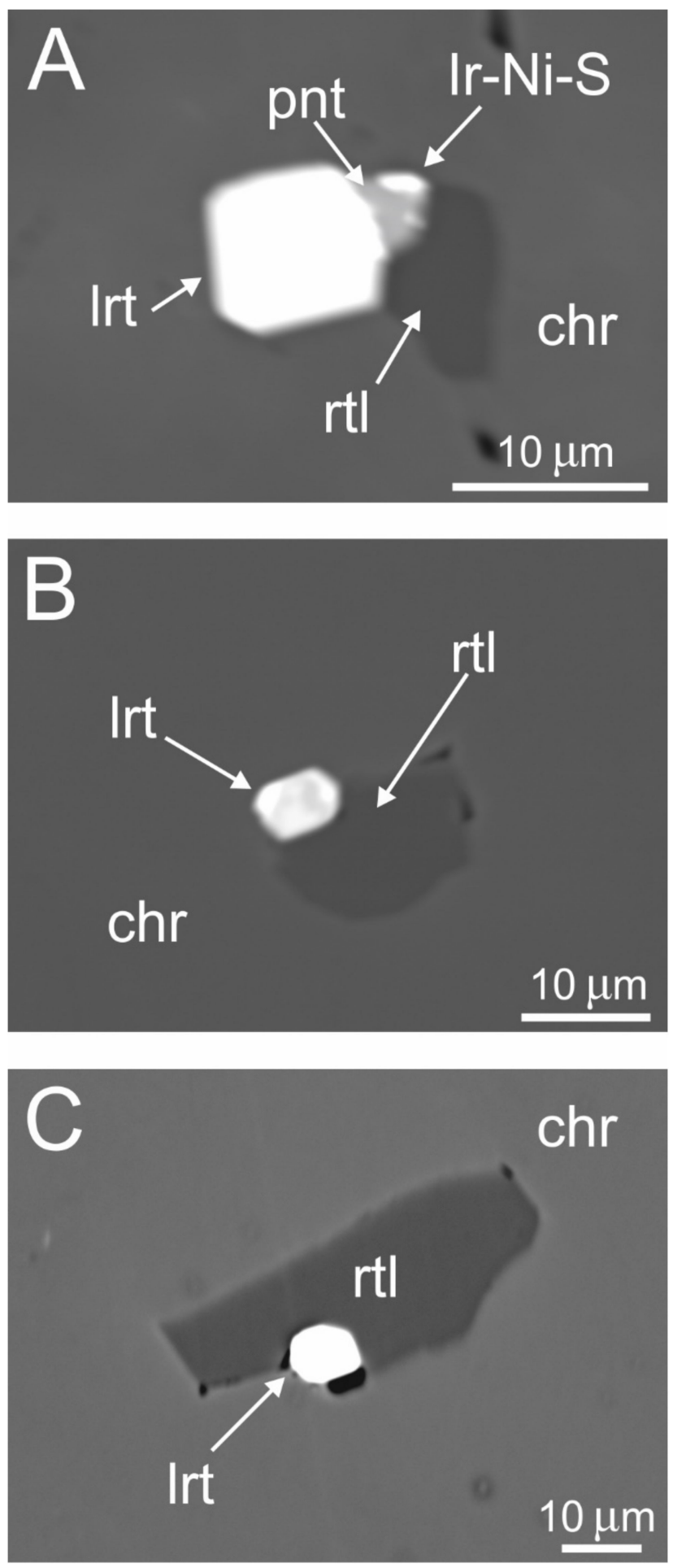

Figure 9. BSE images of rutile-PGM composite inclusions in chromite. Composite grain of rutile, laurite, pentlandite, and Ir-Ni sulfide in fresh chromite of Loma Peguera (A). Rutile-laurite composite inclusions in chromite of Nuasahi (B,C). Abbreviations: lrt, laurite; others as in Figures 1 and 4. 


\section{Trace Element Composition of Rutile from Chromitite}

A total of 328 electron microprobe analyses of trace elements were performed on rutile grains larger than $10 \mu \mathrm{m}$, in which fluorescence effects were absent. Compositions of small rutile inclusions, e.g., the minute needles in Figure $6 \mathrm{C}$, had to be discarded because of the systematic contamination of $\mathrm{Cr}$. Representative compositions of large grains (Table 3 ) show that rutile contains two suites of trace elements, one consisting of chromitite-compatible metals $(\mathrm{Cr}, \mathrm{V}, \mathrm{Al}, \mathrm{Fe}, \mathrm{Mg}, \mathrm{Mn})$, the another represented by high field-strength elements (HFSE), e.g., $\mathrm{Nb}, \mathrm{W}$, and $\mathrm{Zr}$, usually found in rutile from other petrologic assemblages.

Table 3. Trace elements concentration (ppm) and Zr-based crystallization temperature for selected rutile grains in chromitites.

\begin{tabular}{|c|c|c|c|c|c|c|c|c|c|c|}
\hline Element & $\mathrm{Mg}$ & Al & $\mathbf{V}$ & $\mathrm{Cr}$ & Mn & Fe & $\mathrm{Zr}$ & $\mathrm{Nb}$ & $\mathbf{W}$ & $\mathrm{T}^{\circ} \mathrm{C}$ \\
\hline $\begin{array}{c}\text { Detection } \\
\text { Limit (ppm) }\end{array}$ & 50 & 50 & 50 & 50 & 100 & 50 & 50 & 100 & 150 & \\
\hline & \multicolumn{10}{|c|}{ Bushveld (UG2 chromitite layer) } \\
\hline UG2A-52 & 12 & 3710 & 14,806 & 6021 & 178 & & 1096 & & 8 & 743 \\
\hline UG2A-56 & & 3832 & 14,888 & 6062 & 85 & 23 & 1510 & 112 & & 776 \\
\hline UG2A-79 & & 3615 & 14,561 & 6014 & & 62 & 1118 & 245 & 71 & 745 \\
\hline UG2B-23 & & 3832 & 16,546 & 5870 & 85 & 163 & 1806 & 566 & & 795 \\
\hline UG2B-24 & & 3372 & 16,743 & 5781 & 85 & 187 & 2199 & 531 & & 818 \\
\hline UG2B-25 & & 3435 & 16,587 & 5946 & & 194 & 1962 & 517 & 95 & 805 \\
\hline UG2B-26 & 12 & 3504 & 16,288 & 5918 & 39 & 257 & 1777 & 587 & & 794 \\
\hline UG2B-27 & 24 & 3705 & 16,179 & 6226 & 31 & 358 & 1836 & 496 & & 797 \\
\hline UG2B-28 & & 3377 & 16,336 & 7526 & 62 & 637 & 2073 & 419 & & 811 \\
\hline UG2B-29 & & 3763 & 16,764 & 6705 & 62 & 334 & 2102 & 440 & & 812 \\
\hline UG2B-31 & & 3684 & 14,561 & 5747 & 108 & 155 & 725 & & 63 & 703 \\
\hline UG2B-32 & & 3393 & 13,963 & 5699 & 62 & 117 & 800 & 98 & & 712 \\
\hline UG2B-33 & & 3657 & 14,344 & 5857 & 54 & 62 & 792 & 126 & 40 & 711 \\
\hline UG2B-34 & & 3837 & 14,357 & 5994 & 70 & 140 & 755 & 28 & 270 & 707 \\
\hline \multirow[t]{2}{*}{ UG2B-47 } & 18 & 3763 & 14,813 & 6445 & 23 & & 392 & 63 & & 650 \\
\hline & \multicolumn{10}{|c|}{ Bushveld (Merensky Reef) } \\
\hline MR1-23 & 6 & 439 & 10,788 & 6267 & 23 & 1710 & 3368 & 440 & & 869 \\
\hline MR1-25 & & 492 & 10,591 & 6137 & & 1663 & 1414 & & 79 & 769 \\
\hline MR1-27 & & 550 & 11,930 & 5665 & & 1601 & 1481 & 126 & 71 & 774 \\
\hline MR1-28 & 54 & 445 & 10,442 & 6295 & 124 & 2402 & 474 & 7 & & 666 \\
\hline MR1-29 & 24 & 535 & 11,156 & 4488 & & 808 & 1333 & 126 & & 763 \\
\hline MR1-31 & & 492 & 11,298 & 4167 & 70 & 871 & 1362 & 315 & & 765 \\
\hline MR1-32 & 18 & 492 & 11,366 & 3318 & & 925 & 859 & 119 & & 719 \\
\hline MR1-33 & & 487 & 11,727 & 4543 & 15 & 342 & 874 & 14 & & 721 \\
\hline MR2-1 & & 434 & 11,122 & 3435 & 39 & 1982 & 955 & 601 & & 729 \\
\hline MR2-4 & & 460 & 11,842 & 3962 & 46 & 979 & 1310 & 356 & 135 & 761 \\
\hline MR2-11 & 30 & 466 & 11,910 & 4872 & 70 & 1469 & 363 & & & 643 \\
\hline MR2-13 & & 429 & 10,530 & 3873 & & 832 & 733 & 189 & 48 & 704 \\
\hline MR2-18 & 42 & 402 & 11,346 & 3797 & & 373 & 407 & & & 653 \\
\hline MR2-19 & 12 & 476 & 11,359 & 3606 & & 482 & 585 & 126 & & 684 \\
\hline \multirow[t]{2}{*}{ MR2-22 } & & 471 & 11,557 & 5357 & 62 & 1772 & 466 & 203 & 95 & 664 \\
\hline & \multicolumn{10}{|c|}{ Jacurici (ultramafic sill) } \\
\hline JC135-1 & 12 & 550 & 5384 & 4995 & 101 & 1228 & 1029 & 1950 & 745 & 737 \\
\hline JC135-2 & & 556 & 4935 & 5433 & 147 & 1026 & 1096 & 895 & 611 & 743 \\
\hline JC135-9 & & 508 & 4969 & 4906 & 93 & 824 & 1066 & 1209 & 270 & 740 \\
\hline IC135-11 & 30 & 482 & 4942 & 4694 & 62 & 1026 & 874 & 601 & 325 & 721 \\
\hline JC135-13 & & 545 & 4929 & 5768 & 85 & 1454 & 1392 & 1803 & 174 & 767 \\
\hline JC148-2 & & 556 & 5336 & 4393 & 147 & 1889 & 1251 & 105 & 1523 & 756 \\
\hline JC148-3 & & 519 & 5248 & 4187 & 54 & 4229 & 1436 & 273 & 1253 & 771 \\
\hline JC148-5 & 30 & 508 & 5472 & 4119 & 93 & 3187 & 1273 & & 1808 & 758 \\
\hline JC148-6 & & 550 & 5051 & 5761 & 186 & 2106 & 903 & 63 & 952 & 724 \\
\hline JC148-8 & 18 & 540 & 5330 & 5323 & 186 & 1547 & 866 & & 1158 & 720 \\
\hline JC150-1 & 18 & 524 & 5309 & 4297 & 116 & 482 & 1673 & 419 & 1340 & 787 \\
\hline JC150-3 & 12 & 545 & 5282 & 3982 & 139 & 233 & 1740 & 706 & 872 & 791 \\
\hline JC150-5 & 12 & 487 & 4765 & 4208 & 70 & 575 & 1688 & 98 & 1094 & 788 \\
\hline JC150-6 & & 508 & 4711 & 4119 & 108 & 521 & 2028 & 287 & 642 & 808 \\
\hline JC150-10 & 30 & 540 & 4786 & 5022 & 46 & 396 & 1710 & 2020 & 920 & 789 \\
\hline
\end{tabular}


Table 3. Cont

\begin{tabular}{|c|c|c|c|c|c|c|c|c|c|c|}
\hline Element & $\mathrm{Mg}$ & Al & $\mathbf{V}$ & $\mathrm{Cr}$ & Mn & Fe & Zr & $\mathrm{Nb}$ & $\mathbf{W}$ & $\mathbf{T}^{\circ} \mathrm{C}$ \\
\hline $\begin{array}{c}\text { Detection } \\
\text { Limit (ppm) }\end{array}$ & 50 & 50 & 50 & 50 & 100 & 50 & 50 & 100 & 150 & \\
\hline & \multicolumn{10}{|c|}{ Loma Peguera (chromitite pod in mantle tectonite) } \\
\hline LP4-2 & 935 & 683 & 12,705 & 12,665 & 101 & 13,805 & 681 & & & 698 \\
\hline LP4-4 & 434 & 720 & 11,713 & 13,527 & 527 & 12,942 & 659 & 168 & & 695 \\
\hline LP4-5 & 368 & 715 & 12,692 & 14,088 & 658 & 17,458 & 703 & 140 & & 700 \\
\hline LP4-6 & 1387 & 667 & 11,958 & 13,930 & 1139 & 43,521 & 341 & & & 638 \\
\hline LP4-8 & 1694 & 540 & 11,026 & 13,335 & 1929 & 60,179 & 333 & 28 & 119 & 636 \\
\hline LP4-9 & 1037 & 672 & 11,808 & 13,828 & 813 & 34,909 & 629 & 126 & 190 & 690 \\
\hline LP4-10 & 1538 & 582 & 11,563 & 12,275 & 2347 & 56976 & 785 & 42 & 278 & 711 \\
\hline LP4-11 & 706 & 662 & 12,318 & 10,160 & 558 & 23,653 & 1022 & 91 & & 736 \\
\hline LP4-12 & 103 & 619 & 12,107 & 10,482 & 132 & 6187 & 837 & 91 & 79 & 717 \\
\hline LP4-13 & 199 & 704 & 11,937 & 10,201 & 426 & 15,095 & 1073 & 7 & 48 & 741 \\
\hline LP4-14 & 283 & 693 & 11,727 & 12,678 & 209 & 12110 & 896 & 77 & & 723 \\
\hline LP6-1 & & 746 & 11,910 & 12,302 & 147 & 1547 & 792 & & & 711 \\
\hline LP6-2 & & 699 & 12,162 & 12,706 & 15 & 2309 & 814 & 35 & & 714 \\
\hline LP6-3 & 253 & 704 & 11,992 & 11,228 & 132 & 11038 & 1081 & 21 & & 742 \\
\hline \multirow[t]{2}{*}{ LP6-4 } & 416 & 672 & 11,060 & 11,495 & 132 & 10,952 & 1051 & & 16 & 739 \\
\hline & \multicolumn{10}{|c|}{ Nuasahi chromitite fragment Breccia Zone } \\
\hline NS9-4 & & 535 & 6064 & 5864 & 77 & 1562 & 74 & 14 & 1483 & 530 \\
\hline NS9-15 & & 460 & 6050 & 5706 & 85 & 536 & 192 & 77 & 238 & 595 \\
\hline NS9-21 & & 550 & 5887 & 6028 & 23 & 855 & 148 & 35 & 1491 & 576 \\
\hline NS9-23 & & 476 & 5975 & 5980 & 217 & 816 & 207 & 133 & 1301 & 600 \\
\hline NS9-24 & & 476 & 5670 & 6493 & 124 & 668 & 141 & 28 & 1871 & 572 \\
\hline NS9-30 & 12 & 503 & 6016 & 6213 & 240 & 731 & 81 & 14 & 1308 & 536 \\
\hline NS9-40 & & 535 & 5894 & 5994 & 85 & 1586 & 74 & 35 & 809 & 530 \\
\hline NS9-48 & & 482 & 6207 & 6014 & 155 & 917 & 67 & 21 & 357 & 524 \\
\hline NS9-49 & & 513 & 6186 & 6076 & 101 & 785 & 304 & 28 & 666 & 629 \\
\hline NS9-58 & & 503 & 6003 & 5918 & 178 & 661 & 126 & 28 & 2720 & 565 \\
\hline NS9-60 & & 503 & 6064 & 6247 & 39 & 816 & 237 & 14 & 3196 & 610 \\
\hline NS9-62 & 24 & 460 & 6003 & 7383 & 256 & 505 & 89 & 14 & 7415 & 542 \\
\hline NS9-70 & & 593 & 6084 & 7649 & 93 & 1236 & 244 & 28 & 7549 & 612 \\
\hline NS9-79 & & 503 & 5975 & 7389 & 85 & 497 & 185 & 77 & 7526 & 592 \\
\hline \multirow[t]{2}{*}{ NS9-97 } & & 519 & 6030 & 6938 & 54 & 2161 & 207 & 91 & 2894 & 600 \\
\hline & \multicolumn{10}{|c|}{ Cedrolina chromitite } \\
\hline CD14-6 & 127 & 180 & 4765 & 7985 & & 1656 & 52 & 49 & 198 & 509 \\
\hline CD14-8 & 12 & 132 & 4908 & 9654 & & 1492 & 37 & 126 & 230 & 489 \\
\hline CD47A-1 mtx & 78 & 228 & 4534 & 23,126 & & 560 & 30 & 133 & 555 & 477 \\
\hline CD100-4 & 24 & 138 & 4745 & 14,745 & & 1158 & 67 & 1069 & 1078 & 524 \\
\hline CD100-22 & 96 & 180 & 4629 & 13,431 & & 1104 & 207 & 482 & 1475 & 600 \\
\hline CD100-51 & 48 & 201 & 4929 & 13,246 & & 793 & 96 & 615 & 714 & 547 \\
\hline CD100-69 & 18 & 222 & 4629 & 16,072 & & 1003 & 67 & 447 & 1023 & 524 \\
\hline CD105C-1 & 386 & 344 & 4738 & 23,988 & & 700 & 89 & 238 & 5345 & 542 \\
\hline CD105C-2 & 103 & 254 & 4595 & 26,266 & & 1034 & 74 & 433 & 3640 & 530 \\
\hline CD105C-14 & 96 & 212 & 4847 & 27,532 & & 770 & 30 & 405 & 5273 & 477 \\
\hline CD105C-30 & 90 & 222 & 4704 & 39177 & & 1135 & 89 & 343 & 5091 & 542 \\
\hline CD105C-6mtx & 84 & 265 & 4997 & 26,089 & & 544 & 37 & 308 & 3116 & 489 \\
\hline CD105C-13mtx & 157 & 243 & 5262 & 23,290 & & 474 & 67 & 350 & 3997 & 524 \\
\hline CD105C-50mtx & 145 & 333 & 4704 & 29,133 & & 1749 & 59 & 287 & 4480 & 517 \\
\hline CD105C-51mtx & 157 & 217 & 4643 & 23105 & & 653 & 96 & 224 & 2435 & 547 \\
\hline
\end{tabular}

The labels refer to single rutile grains. $\mathrm{T}^{\circ} \mathrm{C}=$ temperature calculated; for a pressure of $0.5 \mathrm{GPa}$ using the calibration of [12]. "mtx" $=$ rutile grain in silicate matrix. Concentrations lower; than detection limit are semiquantitative. Empty box $=$ element absent.

Chromium is the most abundant trace element in rutile from all types of chromitite. The highest concentrations are found in rutile from $\mathrm{LP}(\mathrm{Cr}=10,160-14,211 \mathrm{ppm})$ and $\mathrm{CD}(\mathrm{Cr}=7567-39,177 \mathrm{ppm})$ (Table 4), where the host chromite has the highest $\mathrm{Cr}_{2} \mathrm{O}_{3}$ contents. The other chromitites contain rutile with $\mathrm{Cr}$ contents varying in the range of 3318-8142 ppm. High Cr concentrations were occasionally observed in rutile grains within the silicate matrix, confirming that $\mathrm{Cr}$ was not related to secondary fluorescence from chromite. Among the other chromitite-compatible elements, both magnesium and manganese were detected sporadically in $18 \%$ and $24 \%$ of the rutile analyses, respectively. Average $\mathrm{Mg}$ concentrations up to $244 \mathrm{ppm}$ and $719 \mathrm{ppm}$ characterize rutile from CD and LP, respectively, and are consistently close to or below detection limit in all the other occurrences. Manganese is close to the detection limit or zero in rutile from all the localities, 
except for LP, where rutile contains up to $2347 \mathrm{ppm}$ Mn. Iron concentrations above detection limit were encountered in $95 \%$ of the rutile analyses. The average iron content is always high in rutile from LP, but, excluding two anomalous samples from NS, concentrations decrease down to 1398-196 ppm in rutile from NS, JC, MR, CD, and UG2. Aluminum is found in all rutile analyses, with average concentrations of a few hundred ppm in MR (465), JC (519), LP (669), NS (515), and CD (224), and as high as 3665 ppm in UG2 (Table 4).

Table 4. Statistics of trace element concentration (ppm) and Zr-in-rutile based temperature for 328 rutile analyses.

\begin{tabular}{|c|c|c|c|c|c|c|c|c|c|c|}
\hline & Mg & Al & $\mathbf{V}$ & $\mathrm{Cr}$ & Mn & Fe & Zr & $\mathrm{Nb}$ & $\mathbf{W}$ & $\mathrm{T}^{\circ} \mathrm{C}$ \\
\hline $\begin{array}{c}\text { Detection } \\
\text { Limit (ppm) }\end{array}$ & 50 & 50 & 50 & 50 & 100 & 50 & 50 & 100 & 150 & \\
\hline \multicolumn{11}{|c|}{ Bushveld UG2 chromitite layer (2 samples, 45 analyses) } \\
\hline Min & 6 & 3372 & 13,963 & 5617 & 23 & 8 & 200 & 28 & 8 & 597 \\
\hline Max & 24 & 3874 & 16,764 & 7526 & 178 & 637 & 2199 & 587 & 270 & 818 \\
\hline Average & 13 & 3665 & 15,148 & 6059 & 59 & 152 & 904 & 242 & 108 & 703 \\
\hline \multicolumn{11}{|c|}{ Bushveld Merensky Reef (2 samples, 38 analyses) } \\
\hline Min & 6 & 402 & 10,292 & 3318 & 15 & 303 & 81 & 7 & 32 & 536 \\
\hline Max & 54 & 566 & 12,182 & 6438 & 147 & 2402 & 3368 & 909 & 135 & 869 \\
\hline Average & 23 & 465 & 11,316 & 4512 & 56 & 1035 & 861 & 283 & 72 & 698 \\
\hline \multicolumn{11}{|c|}{ Jacurici ultramafic sill (3 samples, 32 analyses) } \\
\hline Min & 12 & 471 & 4684 & 3982 & 15 & 218 & 444 & 63 & 8 & 660 \\
\hline Max & 30 & 556 & 5472 & 5768 & 318 & 4229 & 2028 & 2111 & 1808 & 808 \\
\hline Average & 20 & 519 & 5078 & 4708 & 90 & 1168 & 1201 & 879 & 768 & 747 \\
\hline \multicolumn{11}{|c|}{ Loma Peguera chromitite pod in mantle tectonite ( 2 samples, 17 analyses) } \\
\hline Min & 30 & 540 & 11,026 & 10,160 & 15 & 1547 & 333 & 7 & 16 & 636 \\
\hline Max & 1694 & 746 & 13,147 & 14,211 & 2347 & 60,179 & 1081 & 168 & 278 & 742 \\
\hline Average & 630 & 669 & 11,947 & 12,438 & 455 & 19,621 & 766 & 75 & 122 & 705 \\
\hline \multicolumn{11}{|c|}{ Nuasahi chromitite fragment Breccia Zone (1 sample, 95 analyses) } \\
\hline Min & 6 & 439 & 5670 & 5439 & 101 & 241 & 7 & 7 & 222 & 408 \\
\hline Max & 84 & 916 & 6492 & 8142 & 356 & 30,905 & 511 & 280 & 7549 & 672 \\
\hline Average & 23 & 515 & 6033 & 6503 & 98 & 1398 & 248 & 52 & 3190 & 602 \\
\hline \multicolumn{11}{|c|}{ Cedrolina chromitite (5 samples, 101 analyses) } \\
\hline Min & 6 & 111 & 4432 & 7567 & & 272 & 7 & 49 & 24 & 408 \\
\hline Max & 2726 & 1461 & 5370 & 39,177 & & 3148 & 540 & 1782 & 6836 & 677 \\
\hline Average & 156 & 224 & 4787 & 19,157 & & 954 & 135 & 394 & 2592 & 546 \\
\hline
\end{tabular}

$\mathrm{DL}=$ detection limit. Min-Max-Average = minimum-maximum concentrations; including values below detection limit.

Vanadium is the second most abundant trace element in rutile (Tables 3 and 4), showing the highest concentrations in the magmatic chromitites of UG2 (V = 13963-16764 ppm), MR ( $\mathrm{V}=10292-12182 \mathrm{ppm})$, and LP $(\mathrm{V}=11026-13147 \mathrm{ppm})$. The magmatic chromite of JC contains vanadium-poor rutile with concentrations ( $\mathrm{V}=4684-5472 \mathrm{ppm})$ intermediate between those in the metamorphic deposits of NS ( $\mathrm{V}=5670-6490 \mathrm{ppm})$ and $\mathrm{CD}(\mathrm{V}=4432-5370 \mathrm{ppm})$. The $\mathrm{V}$ content of rutile in general correlates with the $\mathrm{Fe}_{2} \mathrm{O}_{3}$ of the host chromite, suggesting that it was incorporated in the rutile as $\mathrm{V}^{4+}$, under a relatively high oxidation rate.

Niobium was found in $70 \%$ of the rutile analyses varying from below the detection limit (100 ppm) up to more than $2000 \mathrm{ppm}$. This wide range is covered by rutile from stratiform chromitites showing maximum $\mathrm{Nb}$ concentrations up to $587 \mathrm{ppm}$ in UG2, 
$601 \mathrm{ppm}$ in MR, and $2020 \mathrm{ppm}$ in JC. Rutile has the lowest $\mathrm{Nb}$ content at LP ( $<168 \mathrm{ppm})$, and NS ( $<280 \mathrm{ppm}$ ) (Tables 3 and 4$)$. Relatively high $\mathrm{Nb}$ up to $1780 \mathrm{ppm}$ is found in a few samples of the CD deposit, although $92 \%$ of the analyses have less than $615 \mathrm{ppm} \mathrm{Nb}$ (Figure 10B).
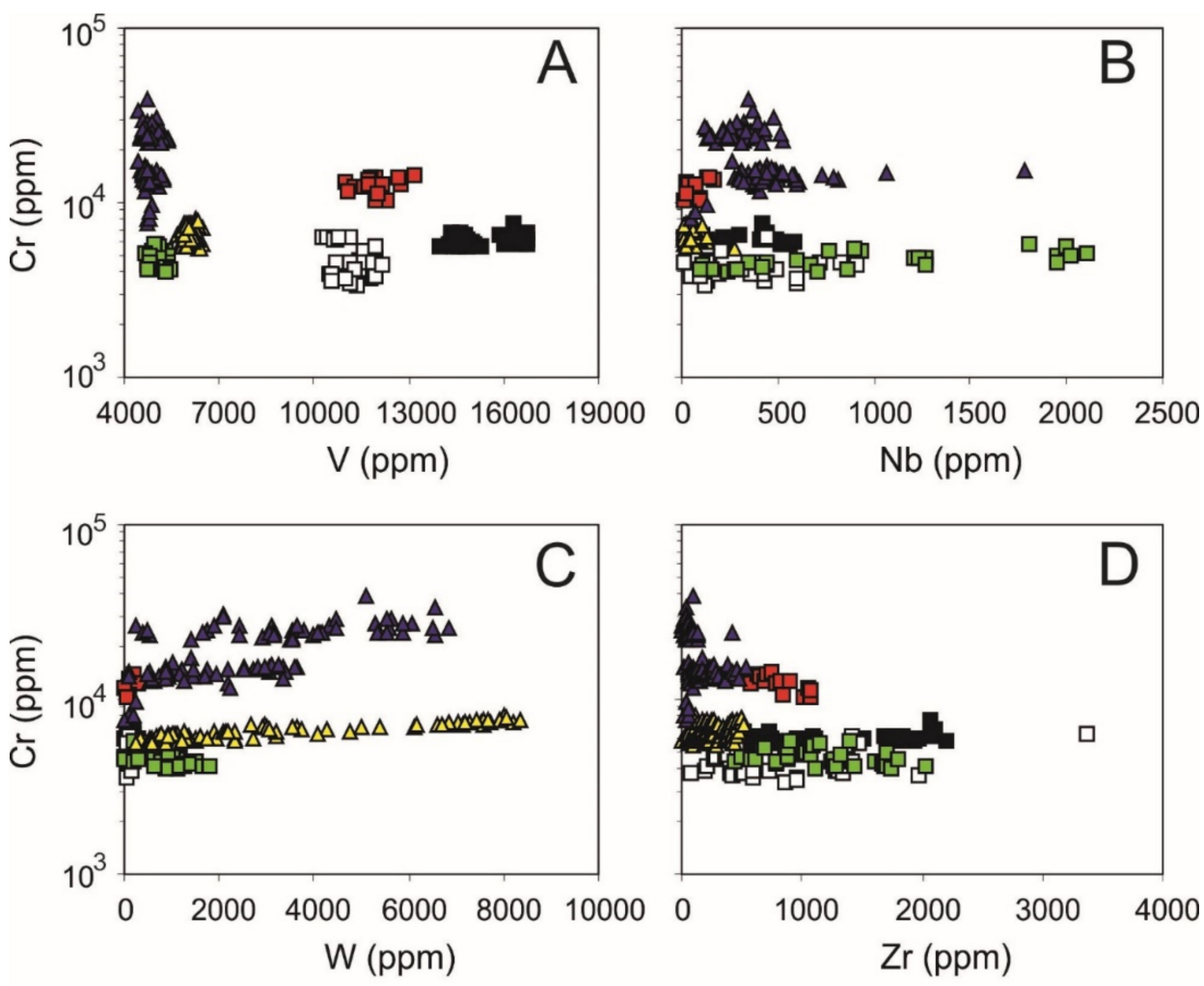

$$
\begin{aligned}
& \text { Magmatic chromitites: } \\
& \text { - UG }=\text { UG2 } \\
& \square \text { MR }=\text { Merensky Reef } \\
& \square \mathrm{JC}=\text { Jacurici } \\
& \square \mathrm{LP}=\text { Loma Peguera }
\end{aligned}
$$

Metamorphic chromitites:

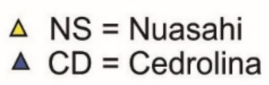

Figure 10. Trace element $(\mathrm{V}, \mathrm{Nb}, \mathrm{W}, \mathrm{Zr})$ variations versus $\mathrm{Cr}$ in accessory rutile from chromitite (concentrations in ppm). Cr versus $\mathrm{V}$ (A); Cr versus $\mathrm{Nb}$ (B); $\mathrm{Cr}$ versus $\mathrm{W}$ (C); $\mathrm{Zr}$ versus $\mathrm{Cr}$ (D).

Wolfram was detected in $76 \%$ of rutile analyses, varying from close to detection limit (UG2, MR, LP) to $1808 \mathrm{ppm}$ (JC), and maximum concentrations of $8334 \mathrm{ppm}$ and $6836 \mathrm{ppm}$ in NS and CD, respectively (Table 4).

Zirconium concentrations above detection limit were encountered in $92 \%$ of rutile analyses. The highest concentrations are found in rutile from UG2, MR, JC, and LP, decreasing from $3368 \mathrm{ppm}$ to $1081 \mathrm{ppm}$ (Table 4). Rutile from the chromitites of NS and $\mathrm{CD}$ is the most depleted in $\mathrm{Zr}$, with average contents of $277 \mathrm{ppm}$ and $165 \mathrm{ppm}$, respectively, and maximum values not exceeding $511 \mathrm{ppm}$ and $540 \mathrm{ppm}$, respectively.

The variation patterns of $\mathrm{V}, \mathrm{Nb}, \mathrm{W}$, and $\mathrm{Zr}$ versus $\mathrm{Cr}$ in rutile (Figure 10) are reflections of the different chromitite type (stratiform versus podiform, and unmetamorphic versus metamorphic). Rutile from the podiform chromitite of LP characterizes for the highest $\mathrm{Cr}$ content, medium $\mathrm{V}$ and $\mathrm{Zr}$ concentrations, and maximum depletion in $\mathrm{W}$, compared with the group of stratiform deposits. The rutile from stratiform chromitites (UG2, MR, $\mathrm{JC}$ ) has the lowest $\mathrm{Cr}$ content, and displays the widest ranges of $\mathrm{V}, \mathrm{Nb}$, and $\mathrm{Zr}$, whereas $\mathrm{W}$ is depleted at the minimum levels similar to LP, except for the JC rutile which shows moderate $\mathrm{W}$ enrichment. Rutile from the metamorphic chromitites (NS, CD) distinguishes for the widest variation of $\mathrm{Cr}$ content and the lowest concentrations of $\mathrm{V}, \mathrm{Nb}$, and $\mathrm{Zr}$, except for five samples from $\mathrm{CD}$ which show $\mathrm{Nb}$ enrichment similar to JC. A striking feature 
of rutile from NS and CD is the trend of anomalous W enrichment that largely exceeds maximum concentrations encountered in the unmetamorphic chromitites.

\section{Zr-Based Rutile Geothermometry}

The temperature of rutile crystallization was calculated with the Zr-based geothermometer of [12] for the 328 analyzed grains. Although the authors warn about the use of the $\mathrm{Zr}$-in-rutile thermometer in $\mathrm{SiO}_{2}$ undersatured systems (e.g., chromitite), [13,14] have shown that application to chromitite provides reasonable results in good agreement with geological and petrogenetic conditions. According to these authors, the influence of pressure on calculated temperature is limited to values below $35^{\circ} \mathrm{C}$; therefore, a pressure of 0.5 GPa was selected to treat all the rutile analyses. The results obtained in this contribution are presented in Table 3 and cover distinct thermal ranges at each locality: UG-2 $=597-818^{\circ} \mathrm{C}$ (av. $\left.703^{\circ} \mathrm{C}\right), \mathrm{MR}=536-869^{\circ} \mathrm{C}\left(\right.$ av. $\left.698^{\circ} \mathrm{C}\right), \mathrm{JC}=660-808^{\circ} \mathrm{C}\left(\right.$ av. $\left.747{ }^{\circ} \mathrm{C}\right), \mathrm{LP}=615-742^{\circ} \mathrm{C}$ (av. $705^{\circ} \mathrm{C}$ ), NS $=408-672{ }^{\circ} \mathrm{C}\left(\right.$ av. $\left.602^{\circ} \mathrm{C}\right)$, and $\mathrm{CD}=408-677^{\circ} \mathrm{C}\left(\right.$ av. $\left.546^{\circ} \mathrm{C}\right)$. Temperatures recorded in magmatic (UG2, MR, JC, LP) and metamorphic chromitites (NS,CD) yielded average values of $712{ }^{\circ} \mathrm{C}$ and $573{ }^{\circ} \mathrm{C}$, respectively, representing a gap of about $140{ }^{\circ} \mathrm{C}$. Frequency distribution (Figure 11) shows that there is a wide range of overlapping especially due to the fact that temperatures calculated for rutile in the magmatic chromitite of MR extend from $672{ }^{\circ} \mathrm{C}$ (maximum temperature of NS) downwards to a minimum of $536^{\circ} \mathrm{C}$.
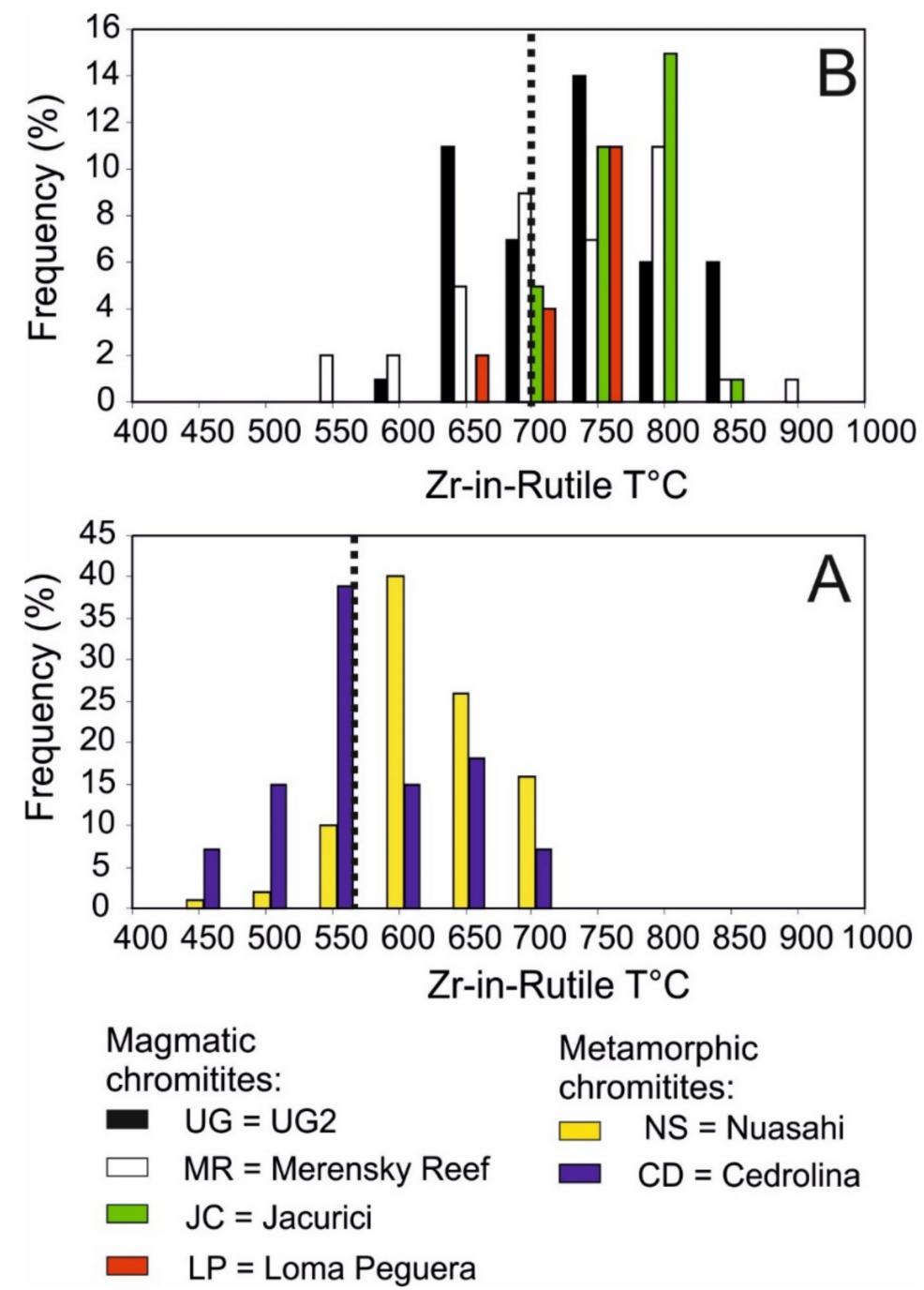

Figure 11. Frequency percent of Zr-in-rutile temperatures (degrees Celsius) calculated with the equation of [12] on a total of 328 grains. Thermal range and average (dotted line) of rutile in magmatic chromitites (A). Thermal range and average (dotted line) of rutile in metamorphic chromitite (B). 


\section{Discussion and Conclusions}

\subsection{Significance of Rutile-Chromite Textural Relations}

Rutile is not a typical accessory mineral in igneous ultramafic contests such as chromitite (Cr ore composed of more than $50 \%$ chromite). However, as this study demonstrates, chromitite crystallized from mantle-derived magmas in particular geological settings may become mineralized with rutile. The accessory rutile in chromitite has distinctive textural setting that allows us to speculate on the origin of rutile in the different types of host chromitite, and to establish the petrogenetic pathways from primary magmatic deposition up to post-magmatic metamorphism.

In chromitites devoid of significant metamorphic overprint (UG2, MR, JC, LP), primary textural relations (Figure 5) indicate that rutile has crystallized either in the intercumulus spaces of chromite or at the rims of chromite grains, precipitating directly from the melt that forms the interstitial silicate matrix of chromitite. This type of rutile can be considered as co-magmatic with chromite, although later in the order of crystallization (magmatic rutile). The pegmatoidal nature of the MR cumulate unit suggests that the reef developed by magma-mixing under high partial pressure of volatiles. Residual fluids released during early solidification of the interstitial melt were forced to percolate upwards and laterally, diffusing along crystal boundaries and intercumulus spaces, possibly driven by thermal and pressure gradient. In their migration, they mixed with trapped interstitial liquid, becoming enriched in $\mathrm{Zr}$ and $\mathrm{Nb}$, which were incorporated in rutile precipitating in the last stage of crystallization (Figure 5E,F). This mechanism enhanced by high fluid activity might have delayed interstitial precipitation of rutile well below the solidus temperature of chromite. Another type of rutile occurring as small needles or euhedral to subhedral crystals included in the core of chromite grains (Figure 8) is considered to have unmixed from the chromite in the subsolidus (exsolved rutile). Rutile subsolidus exsolution is common in all types of chromitite, occurring either on post-magmatic cooling, or during syn-metamorphic recrystallization. The composite inclusions of rutile with highly refractory $\mathrm{PGM} \pm$ sulfide \pm silicate (Figure 9 ) may suggest entrapment into chromite at high temperature (early magmatic rutile). The main issue, in this case, concerns the physical state of the various components at the time of entrapment (solid, solid+liquid, or liquid droplet). The polygonal shape of most composite inclusions may indicate engulfment into chromite as a solid or solid+liquid particle.

Magmatic rutile is absent in all samples of metamorphic chromitite (NS, CD). All primary textures have been obliterated and rutile characterized by extremely variable morphology and grain size occur indifferently associated with altered and deformed chromite or engulfed in the altered silicate matrix of the chromitite (Figures 6 and 7). The association with exotic metasomatic minerals, and the frequent appearance of altered chromite included in rutile, indicate an origin by remobilization of primary rutile during metamorphism (secondary-metamorphic rutile).

\subsection{Chromium and Vanadium in Rutile from Magmatic and Metamorphic Chromitites}

Although percentage levels of trace elements such as $\mathrm{Cr}, \mathrm{V}$, and $\mathrm{Nb}$ have been reported [62] for rutiles, the concentrations of $\mathrm{Cr}$ and $\mathrm{V}$ in accessory rutile from the studied chromitites are distinctly high $(2-10 \times)$ when compared with rutile occurring in lithotypes of regular composition, such as metapelites, metabasites, gneisses, and granulites. In these rocks, $\mathrm{Cr}$ and $\mathrm{V}$ do not exceed $5000 \mathrm{ppm}$ and $4000 \mathrm{ppm}$, respectively [2,11]. The systematic $\mathrm{Cr}-\mathrm{V}$-rich nature of rutile from chromitites is a strong indication of the high activity of these metals in the chromite-forming system, then reflects the composition of the host chromite (Figure 12A,B), varying in relation to the magmatic and metamorphic history of the deposit. 

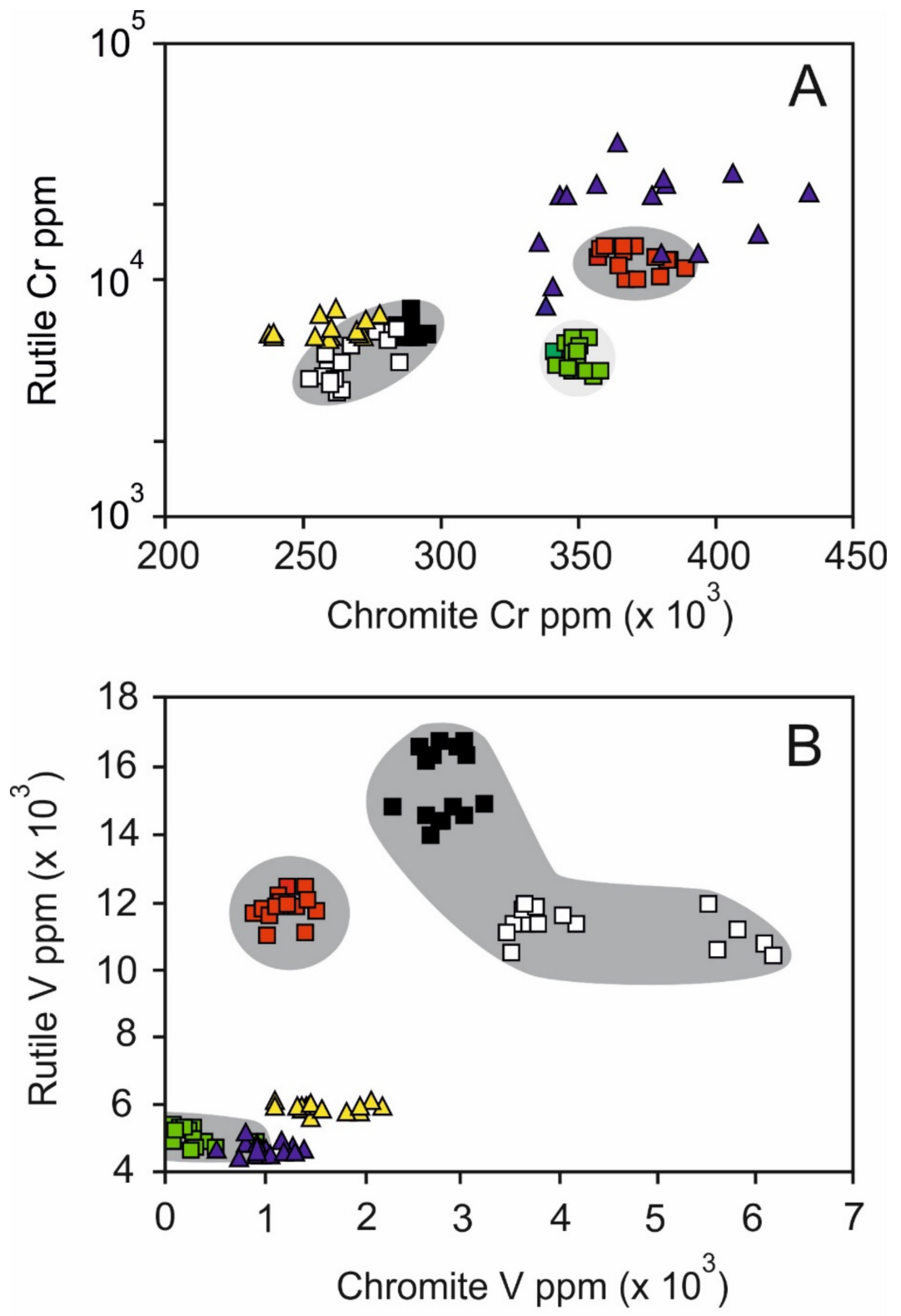

Magmatic chromitites:

Metamorphic chromitites:
UG = UG2
$\square \mathrm{MR}=$ Merensky Reef
$\square \mathrm{JC}=$ Jacurici
- LP = Loma Peguera

$\triangle \mathrm{NS}=$ Nuasahi

$\Delta \mathrm{CD}=$ Cedrolina

Figure 12. Correlation between the $\mathrm{Cr}-\mathrm{V}$ contents in rutile, and $\mathrm{Cr}-\mathrm{V}$ in the host chromite $(\mathbf{A}, \mathbf{B})$. The gray fields emphasize $\mathrm{Cr}-\mathrm{V}$ variations in magmatic chromitite.

Magmatic rutile of LP has crystallized in equilibrium with poorly differentiated high-Cr magma similar to parent melts of podiform chromitite in the upper mantle. The moderate $\mathrm{V}$ content, however, would indicate a more evolved composition compared with SSZ boninite [63]. Magmatic differentiation is responsible for the high- $\mathrm{V}$ and the decrease in $\mathrm{Cr}$ content in rutile of UG2 and MR chromitite layers. In contrast, the low $\mathrm{Cr}-\mathrm{V}$ content of rutile in the JC ultramafic sill is a reflection of the relatively low Cr\# and V-content of chromite that precipitated from poorly differentiated high-Mg magma that prevented $\mathrm{V}$ fractionation and "diluted" its concentration in an overwhelming volume of chromite. 
Complete overprint of the Cedrolina chromitite by multiple regional metamorphic events and metasomatic reactions with hydrous fluids from albite-granite intrusions has obliterated any remnant of magmatic chromite and possible magmatic rutile. Rutile is characterized by a wide range of $\mathrm{Cr}$ content and a constantly low concentration of $\mathrm{V}$. These variation patterns of $\mathrm{Cr}$ and $\mathrm{V}$ in rutile parallel those of the host chromite, supporting genetic heredity. According to [13], rutile formed at the expense of chromite by remobilization of $\mathrm{Ti}, \mathrm{Cr}$, and $\mathrm{V}$ during metamorphism, thus shifting chromite composition towards a "ferrian-chromite" phase characterized by extremely high $\mathrm{Fe}_{2} \mathrm{O}_{3}$, but strongly depleted in Ti and V.

The large rutile crystals observed in the chromitite fragments of the Nuasahi breccia are characterized by a low-Cr, low- $\mathrm{V}$ composition that mimic $\mathrm{Cr}-\mathrm{V}$ relations of the host chromite. Like CD rutile, they are interpreted as secondary crystals formed by re-distribution of $\mathrm{Ti}, \mathrm{Cr}$, and $\mathrm{V}$ during metamorphism. The scarcity of low-temperature hydrous silicates in the chromitite is suggestive of minimal fluid activity, indicating that mobilization of $\mathrm{Ti}, \mathrm{Cr}$, and $\mathrm{V}$ from chromite to form rutile started at temperatures higher than hydrothermal during gabbro intrusion, and continued under the greenschist-amphibolite grade metamorphism [23]. Intrusion of quartz-diorite veins into the chromitite breccia triggered further metasomatic reactions and contamination of the rutile forming system by incompatible elements with felsic signature (see below).

\subsection{Significance of HFSE (Niobium-Zirconium-Wolfram) in Chromitite-Hosted Rutile}

The data in Table 4 show that the total amount of HFSE is very low (av. 963 ppm) in rutile from the podiform chromitite of LP, and is mainly accounted for by Zr. The HFSE content is slightly higher in rutile from the stratiform chromitites of UG2 (av. 1255 ppm) and MR (av. $1216 \mathrm{ppm}$ ), determined by relatively high concentrations of $\mathrm{Zr}+\mathrm{Nb}$. Rutile from the JC ultramafic has the highest concentration of HFSE in the group of magmatic chromitites, determined by equal amounts of $\mathrm{Zr}, \mathrm{Nb}$, and $\mathrm{W}$ summing up to av. $2848 \mathrm{ppm}$. The highest HFSE concentrations are achieved in rutile from metamorphic chromitite of NS (up to $9125 \mathrm{ppm}$ ) and CD (up to $9158 \mathrm{ppm}$ ), being essentially due to the anomalous concentration of $\mathrm{W}$, while both $\mathrm{Zr}$ and $\mathrm{Nb}$ are depleted. Broad positive correlation does exist between $\mathrm{Nb}$ and $\mathrm{Zr}$ in magmatic rutile from the stratiform chromitites of UG2, MR, and JC (Figure 13A), while correlation between $\mathrm{W}$ and $\mathrm{Zr}$ seems to be present only in the samples from JC, although very weak (Figure 13B). No obvious correlation is visible between $\mathrm{Nb}-\mathrm{Zr}, \mathrm{W}-\mathrm{Zr}$, and $\mathrm{W}-\mathrm{Nb}$ in rutile from metamorphic deposits of $\mathrm{NS}$ and $\mathrm{CD}$, which characterize for a trend of exceptional increase in $\mathrm{W}$ content (Figure $13 \mathrm{~B}, \mathrm{C}$ ).

Reciprocal substitutions among HFSE in rutile from magmatic chromitite are presented as compositional fields in the $\mathrm{Zr}-\mathrm{Nb}-\mathrm{W}$ ternary diagram, and compared with data point distribution of the metamorphic chromitites and estimated average composition of depleted mantle, tholeiitic basalt, and continental crust taken from a wide literature (Figure 14).

The Zr-specialized composition observed in magmatic rutile from podiform chromitite (LP) is interpreted as a reflection of the general paucity of lithophile metals $(\mathrm{Nb}, \mathrm{W})$ in magmas parent to chromite deposits in the sub-oceanic upper mantle. On the other hand, it is worthy of remark that the occurrence of rutile in a high-Cr chromitite enriched in $\mathrm{Ti}-\mathrm{V}-\mathrm{Zr}$, and the presence of $\mathrm{Pt}$ minerals at LP [41,42] is irreconcilable with composition and mineral assemblage of high-Cr deposits commonly associated with ophiolites, which are characterized by low Ti-V-Zr, and carry Ru-Os-Ir PGM [63]. Thus, the peculiar mineral chemistry of chromite and rutile at LP requires that the parent melt had relatively high concentration of incompatible elements, implying an origin by partial melting of undepleted mantle source, not consistent with the strongly residual mantle related with SSZ [39]. This raises once again the question of the real geodynamic setting of the Loma Caribe mantle tectonite. 
Magmatic rutile from the stratiform chromitites of Bushveld (UG2, MR) defines a trend of $\mathrm{Nb}$-for-Zr substitution at relatively low $\mathrm{W}$ contents (Figure 14) that is a result of magma mixing and differentiation. As mentioned above, these processes were responsible for the collection and redistribution of $\mathrm{Zr}$ and $\mathrm{Nb}$ in residual melts yielding rutile precipitation in the intercumulus spaces of chromite or at the rims of chromite grains.

The wide range of $\mathrm{Zr}-\mathrm{Nb}$ exchange in rutile of MR was promoted by the high fluid activity in the late residual melts originated by fractional crystallization of the pegmatoid reef. The rutile in the stratiform chromitite of JC defines trends of increasing $\mathrm{Nb}$ and $\mathrm{Zr}$ contents similar to the Bushveld stratiform deposits, (Figure 13A); however, it distinguishes for a sensible and consistent enrichment in $\mathrm{W}$ that causes shifting of rutile compositions towards the Zr-poor field typical of rutile from metamorphic chromitites (Figure 14). Most rutile from the chromitites of NS and CD display systematic low contents of $\mathrm{Zr}$ and $\mathrm{Nb}$, possibly suggesting removal of HFSE from rutile crystallizing under metamorphic conditions. Data point distribution indicates that $\mathrm{Nb}$ was almost totally removed from the rutile of NS, compared with $\mathrm{CD}$, where $\mathrm{Nb}$ appears to decrease gradually. In both cases, the trend of $\mathrm{W}$ substitution for $\mathrm{Zr}$ and $\mathrm{Nb}$ is striking and marks the role of $\mathrm{W}$ as an indicator of the different origin of rutile in magmatic and metamorphic chromitites.
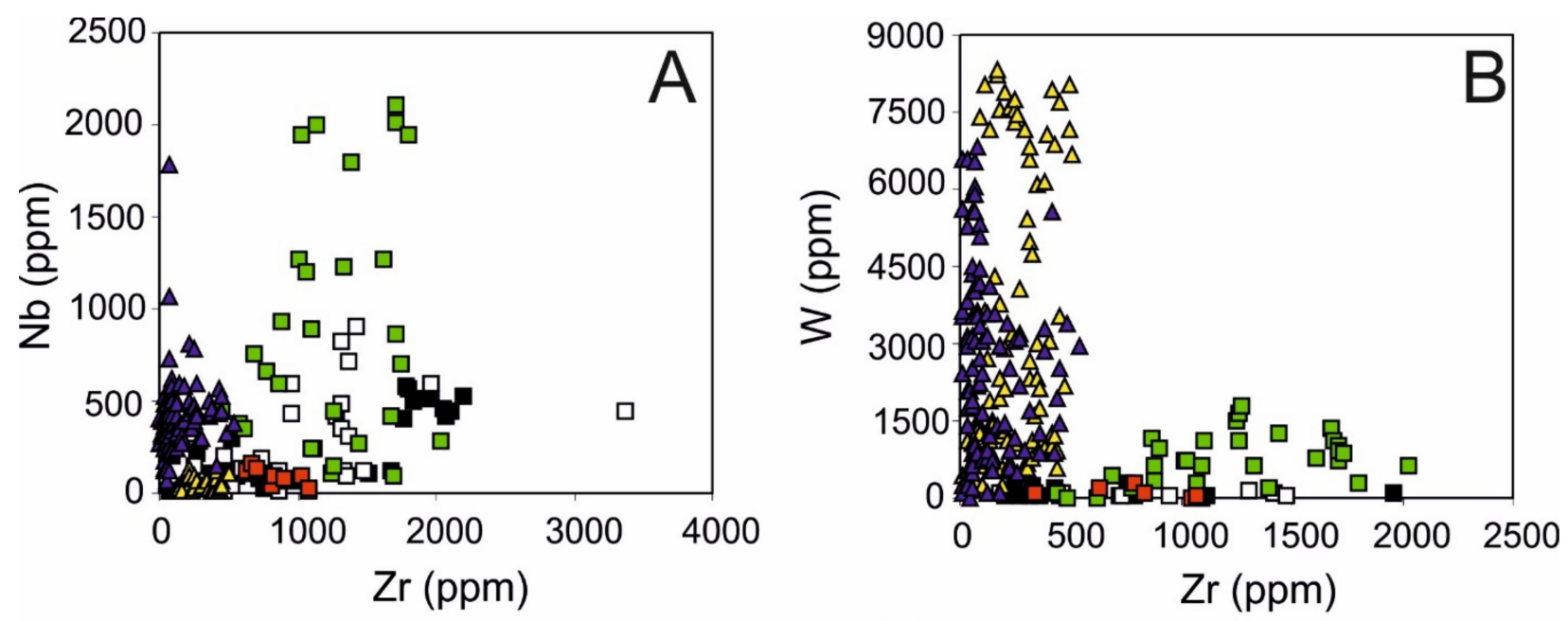

Magmatic chromitites:

- UG $=$ UG2

$\square \mathrm{MR}=$ Merensky Reef

$\square \mathrm{JC}=$ Jacurici

- LP = Loma Peguera

Metamorphic chromitites:

$\triangle \mathrm{NS}=$ Nuasahi

$\Delta \mathrm{CD}=$ Cedrolina

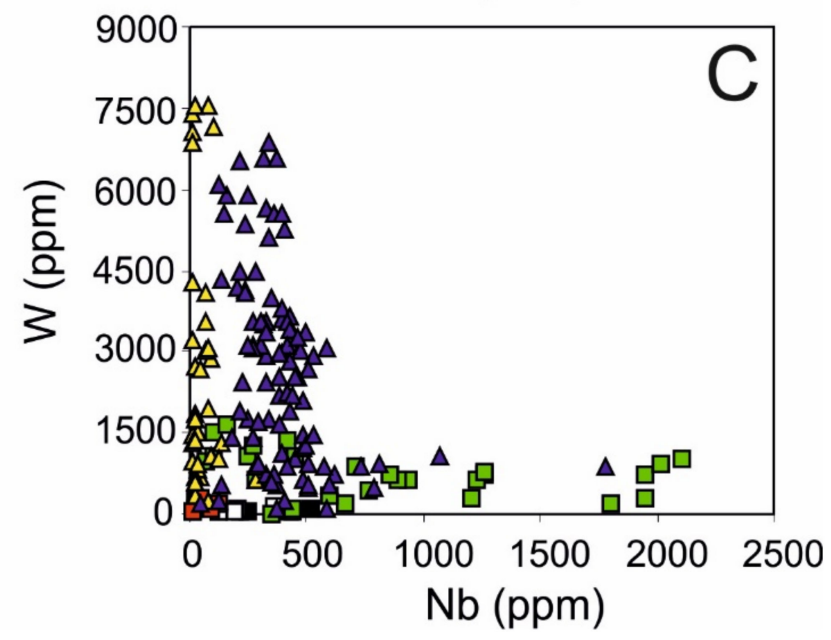

Figure 13. HFS ( $\mathrm{Nb}-\mathrm{Zr}-\mathrm{W})$ interelemental correlation in accessory rutile from chromitite. Enrichment trends of $\mathrm{Nb}$ and $\mathrm{Zr}$ distinguish rutile in magmatic deposits from rutile in metamorphic chromitite (A-C). Anomalous concentrations of $\mathrm{W}$ characterize rutile from metamorphic chromite deposits, in contrast with the magmatic ones (B,C). 


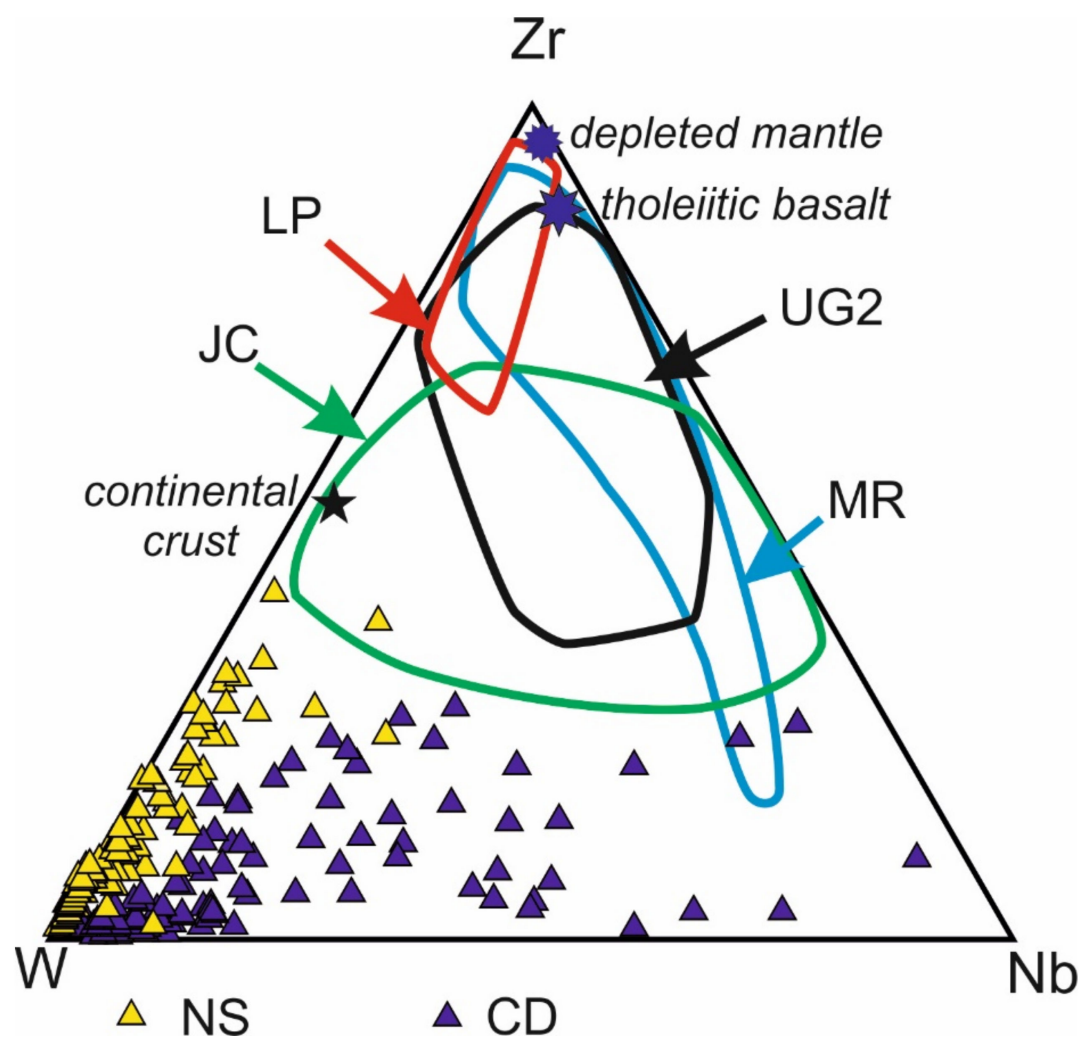

Figure 14. Compositional fields and data point distribution of HFS (Nb-Zr-W) in rutile from the different types of chromitite. Contour color: Red = Loma Peguera (LP), Black = UG2, Blue-Sky = Merensky Reef (MR), Green = Jacurici (JC). Yellow Triangle = Nuasahi. Blue Triangle = Cedrolina . Blue Stars = estimated average composition of depleted mantle, tholeiitic basalt, and continental crust taken from a wide literature.

Geochemical affinity and estimated W abundance in depleted mantle, tholeiitic basalt, and continental crust (Figure 14) predict that $\mathrm{W}$ is generally depleted in mafic magmas derived from mantle partial melting, but has strong tendency to concentrate in melts and fluids of felsic and granitic composition. Therefore, the presence of substantial concentrations of W in ultramafic igneous rocks such as the chromitites must be related with "crustal contamination" processes. The high-W ranges of up to more than $8000 \mathrm{ppm}$ in rutile of $C D$ and NS (Figure 12B,C) argue for the addition of $W$ to the chromitite in the post-magmatic stage, during metamorphism. At Cedrolina, $\mathrm{W}$ was probably part of the incompatible elements assemblage (REE, $\mathrm{U}, \mathrm{Th}, \mathrm{Zr}$ ) that was introduced into the chromitite via infiltrating albite-granite hydrothermal fluids [13]. At Nuasahi, the source of W is uncertain. Considering that the analyzed sample is a chromitite fragment of the breccia [23], W could have been brought into the chromitite by metasomatic reaction with the gabbroic matrix, during intrusion. The reaction was possibly enhanced during the greenschist-amphibolite metamorphic event, and late infiltration of quartz-diorite melt that is likely to have caused the main $\mathrm{W}$ contamination in the chromitite fragments.

Crustal contamination provides a reasonable explanation for the moderate $\mathrm{W}$ enrichment (up to $1808 \mathrm{ppm}$ ) detected in rutile associated with the Jacurici chromitite layer. However, according to currently accepted reconstruction [37], contamination with crustcompatible lithophile elements was not a post-magmatic event at Jacurici, but occurred in the orthomagmatic stage by assimilation of partially melted metasediments bordering the magma chamber. Compositional changes induced in the mafic magma contributed to sustain $\mathrm{Cr}$ saturation long enough for the formation of a thick chromitite layer. There is no evidence of late infiltration of felsic melts in the samples of UG2, MR, and LP chromitite, neither assimilation of country rocks appears to have been effective. The small amounts of 
W encountered in rutile of these magmatic chromitites may be due to the enrichment of incompatible elements into residual melts during magmatic differentiation, or alternatively might have derived from limited contamination in the Bushveld parental magmas during its ascent into the crust [27].

\subsection{Zr-in-Rutile Thermometry of Chromitite}

Results of Zr-in-rutile thermometry indicate that rutile from metamorphic and magmatic chromitites crystallized in diverse thermal ranges (Figure 11). Oscillatory zoning in the studied rutile was not observed, suggesting that the degree of isomorphic substitution of $\mathrm{Zr}$ and other trace elements in rutile associated with chromitite is not related with the growth of the crystals and diffusion rate as reported by [62], but is controlled by the temperature of crystallization.

Correlation of calculated temperatures with the $\mathrm{W}$ content of rutile highlights the existence of a thermal gap of about $140^{\circ} \mathrm{C}$ between the two types of chromitite (Figure 15A). Rutile from both metamorphic chromitites of CD and NS presented average crystallization temperatures of $546{ }^{\circ} \mathrm{C}$ and $602{ }^{\circ} \mathrm{C}$, respectively. These values are in good agreement with the thermal peak of $550-600^{\circ} \mathrm{C}$ proposed for the amphibolite-facies regional metamorphism of the Pilar de Goiás Greenstone Belt [13], and the greenschist-amphibolite metamorphic overprint of the Nuasahi complex [48].

The distribution of data points in the correlation diagram between $\mathrm{W}$ and $\mathrm{T}{ }^{\circ} \mathrm{C}$ poses a maximum thermal limit of $600-650{ }^{\circ} \mathrm{C}$ for the metasomatic enrichment of $\mathrm{W}$ in rutile from metamorphic chromitites. Rutile associated with magmatic chromitites (UG2, MR, LP) is generally W-depleted compared with the metamorphic ones, which apparently exclude contamination from felsic melts of an external source. The Zr-in-rutile thermometry yielded temperatures of magmatic crystallization in the range of $700-869^{\circ} \mathrm{C}$, with some data points extending down to $600{ }^{\circ} \mathrm{C}$ and less. The authors of [14] have shown that rutile from ultramafic cumulates of the Bushveld Critical Zone split into two distinct fields, based on a diagram involving variation of the $\mathrm{Nb}$ content as a function of $\mathrm{Zr}$-in-rutile temperature. These authors interpreted the $\mathrm{Nb}$-rich rutile $(\mathrm{Nb}>4000 \mathrm{ppm})$ as a product of direct crystallization from the mafic magma at temperatures of $800-1000{ }^{\circ} \mathrm{C}$, whereas the $\mathrm{Nb}$-poor rutiles $(\mathrm{Nb}<1000 \mathrm{ppm})$ exsolved from chromite in a subsolidus thermal range of $800-500{ }^{\circ} \mathrm{C}$. According to this model, the great bulk of rutile from chromitite deposits examined in this study would plot in the field of rutile exsolving from chromite at subsolidus temperatures below $800^{\circ} \mathrm{C}$ (Figure 15B).

This conclusion is probably applicable to exsolved rutile scattered in the chromite of magmatic and metamorphic deposits, but disagrees with the origin of magmatic rutile proposed in this work. In magmatic chromitite, rutile exsolution takes place upon cooling in a thermal interval from about $700{ }^{\circ} \mathrm{C}$ down to less than $600{ }^{\circ} \mathrm{C}$. This type of rutile, significantly depleted in $\mathrm{Nb}$, is encountered in both magmatic and metamorphic chromitites (e.g., UG2, MR, LP, CD, NS) (Figure 15B). Rutile needles $(<5 \mu \mathrm{m})$ crystallographically oriented according to the cubic symmetry of the host spinel, are found included in the chromite of metamorphic chromitite (CD, NS). The texture strongly supports exsolution during chromite metamorphism at temperatures well below $600^{\circ} \mathrm{C}$, down to $400^{\circ} \mathrm{C}$.

On the other hand, the occurrence of large rutile grains in the altered matrix of metamorphic chromites is consistent with direct precipitation of rutile from hydrous solutions, thus shifting the temperature of rutile crystallization below the amphibolite facies peak $\left(600-650^{\circ} \mathrm{C}\right)$, but consistent with greenschist facies retrograde metamorphism, as suggested by [13]. The exsolution model is seemingly in contrast with the intercumulus textures of rutile described in the magmatic chromitites which indicates rutile precipitation from the interstitial melt. 

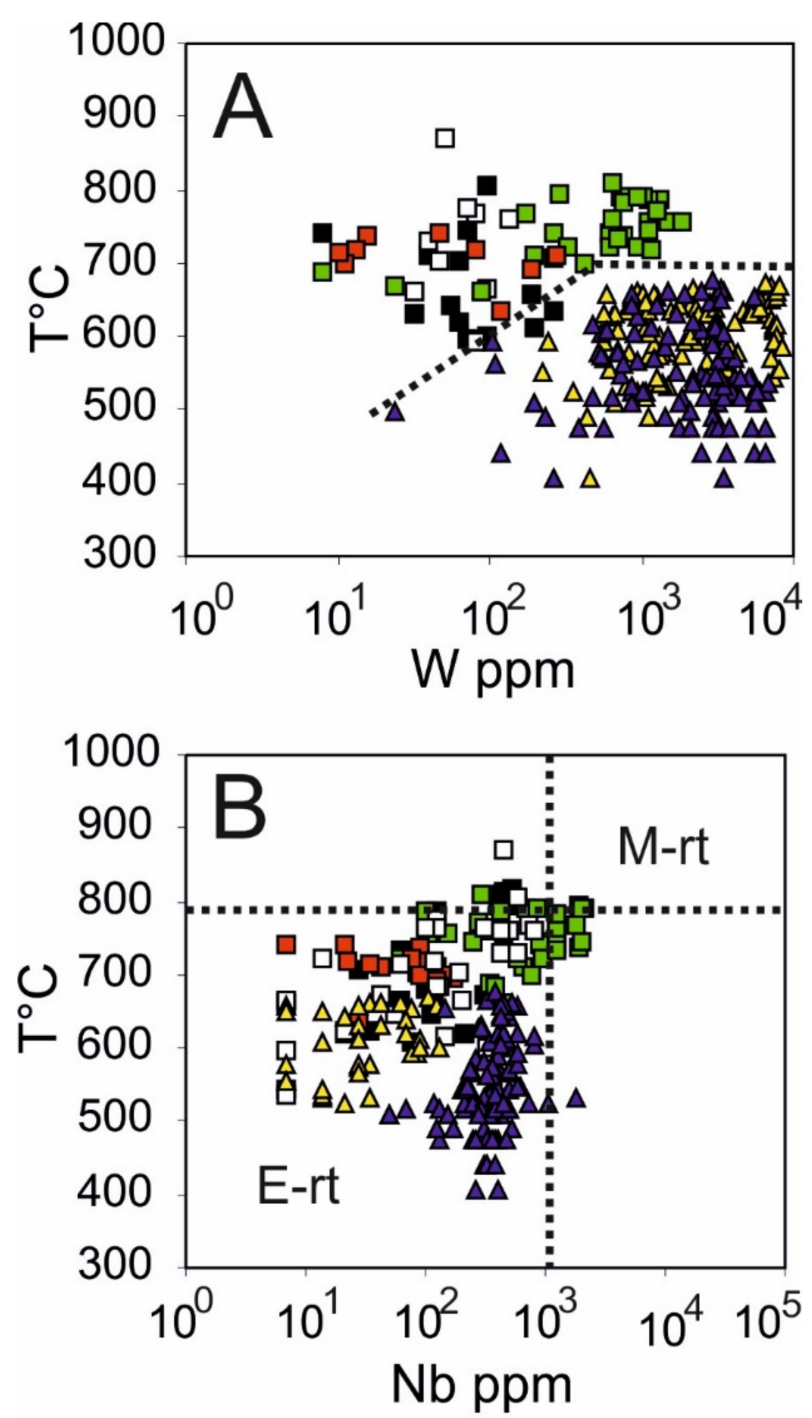

Magmatic chromitites:

- UG = UG2

$\square \mathrm{MR}=$ Merensky Reef

$\mathrm{JC}=$ Jacurici

$\square$ LP $=$ Loma Peguera

Metamorphic chromitites:
$\triangle \mathrm{NS}=$ Nuasahi
$\triangle \mathrm{CD}=$ Cedrolina

Figure 15. Correlation $\mathrm{W}-\mathrm{T}{ }^{\circ} \mathrm{C}$ separates rutile in metamorphic chromitite from rutile in magmatic chromite, the dotted line marking the thermal gap (A). Projection of chromitite-rutile data in the $\mathrm{Nb}-\mathrm{T}^{\circ} \mathrm{C}$ diagram after [14] (B). The dotted cross separates the field of rutile crystallized directly from magmas (M-rt) from the field of rutile exsolved from chromite (E-rt).

On the other hand, the $\mathrm{Nb}$-poor $(\mathrm{Nb}<2000 \mathrm{ppb})$ composition and low crystallization temperature $\left(800-700{ }^{\circ} \mathrm{C}\right)$ would be consistent with rutile exsolution from chromite. It is unlikely that this discrepancy resides in the erroneous calculation of the rutile temperature. Conversely, we believe that these types of rutile are late-magmatic in origin, implying that the thermal interval for magmatic precipitation of rutile that should be extended to temperatures lower than previously supposed. It is worthy of remark that magmatic rutile 
interstitial to chromite in MR appears to have precipitated at temperature quite lower than those proposed by [14] for pure magmatic rutile. We suggest that low crystallization temperatures are to be expected when rutile precipitates from post-chromite interstitial melts enriched in hydrous fluids at relatively high oxygen fugacity, as those reported from MR chromitite in this study.

\subsection{Origin of Rutile-PGM Inclusions in Chromite}

The chromitites of UG2, MR, NS, and LP contain a particular type of polygonal inclusions composed of rutile, PGM, and magmatic sulfides. The rutile-PGM association has been previously reported from a number of chromite deposits associated with layered intrusions $[17,23,56,60,64,65]$. The examples reported in this work regard the chromitites of UG-2, MR, NS, and LP, the latter representing the first documented quotation of rutile PGM inclusions in podiform chromitites of ophiolitic upper mantle.

The presence of PGM inclusions in chromitite is not surprising in consideration of the common association of PGE with chromite. However, the systematic association of PGM with rutile observed in our samples establishes a genetic linkage, and raises again the important question about the origin of rutile: exsolution-related or magmatic precipitation. Previous works have considered the composite inclusions as material crystallized at high temperature in the melt and trapped as solid particles into precipitating chromite. Unfortunately, we were not able to precisely determine the composition of rutile for temperature calculation due to the extremely small size of the grains. Yet, the common euhedral morphology of both rutile and PGM would be consistent with crystallization in a fluid milieu at high temperature. Of particular interest is the occurrence of rutile PGM inclusions observed in the metamorphic chromitite of NS [23] and this work. The association, previously observed in highly metamorphosed chromitites of Brazil [17,56], was ascribed to low temperature remobilization of Ti and PGE during metamorphism and alteration of primary chromite and PGM. In contrast, based on textural relation of the rutile-PGM inclusions in the NS chromitite, [23] concluded that they are relic magmatic minerals endured the metamorphic event. In summary, the rutile PGM inclusions in the magmatic chromitites of UG2, MR, and LP provide further evidence for early deposition of rutile in the pre-chromite stage, an origin that can be extended even to single rutile inclusions allegedly interpreted as chromite exsolution products [14,15].

\subsection{Comparison of Accessory Rutile in Chromitite with Rutile from Other Petrologic Associations}

Accessory rutile associated with chromitite represents a further category of igneous rutile characterized by distinctive trace-element fingerprints dominated by chromitecompatible metals $(\mathrm{Cr}, \mathrm{V})$ and $\operatorname{HFSE}(\mathrm{Nb}, \mathrm{W}, \mathrm{Zr})$. The $\mathrm{Cr}-\mathrm{Nb}$ correlation has been used in the literature to trace the provenance of rutile in sediments, in an attempt to discriminate among rutile originally associated with mafic rocks, metapelites, gneisses, or felsic- and mafic-granulites $[2,7,11]$. Although this approach may lead to contrasting results when dealing with high-grade metamorphic assemblages, we found it useful to illustrate the unusual $\mathrm{Cr} / \mathrm{Nb}$ composition of accessory rutile from chromitites. Using the fields drawn by [7], we have compiled a modified version of the diagram $\mathrm{Cr} / \mathrm{Nb}$, extending the $\mathrm{Cr}$ scale upwards in order to include composition of rutiles from chromitites (Figure 16). The bulk of our analyses cluster in the $\mathrm{Cr}$-rich, $\mathrm{Nb}$-poor sector of the diagram, with only limited overlap with $\mathrm{Cr}$-poor rutile from mafic and metapelitic rocks characterized by high $\mathrm{Nb}$ contents $[7,11]$. The $\mathrm{Cr}$ and $\mathrm{V}$ contents increase according to the composition of the host chromite, establishing a genetic linkage with either the parental magma of the deposit, or compositional reworking of chromite during metamorphism. 


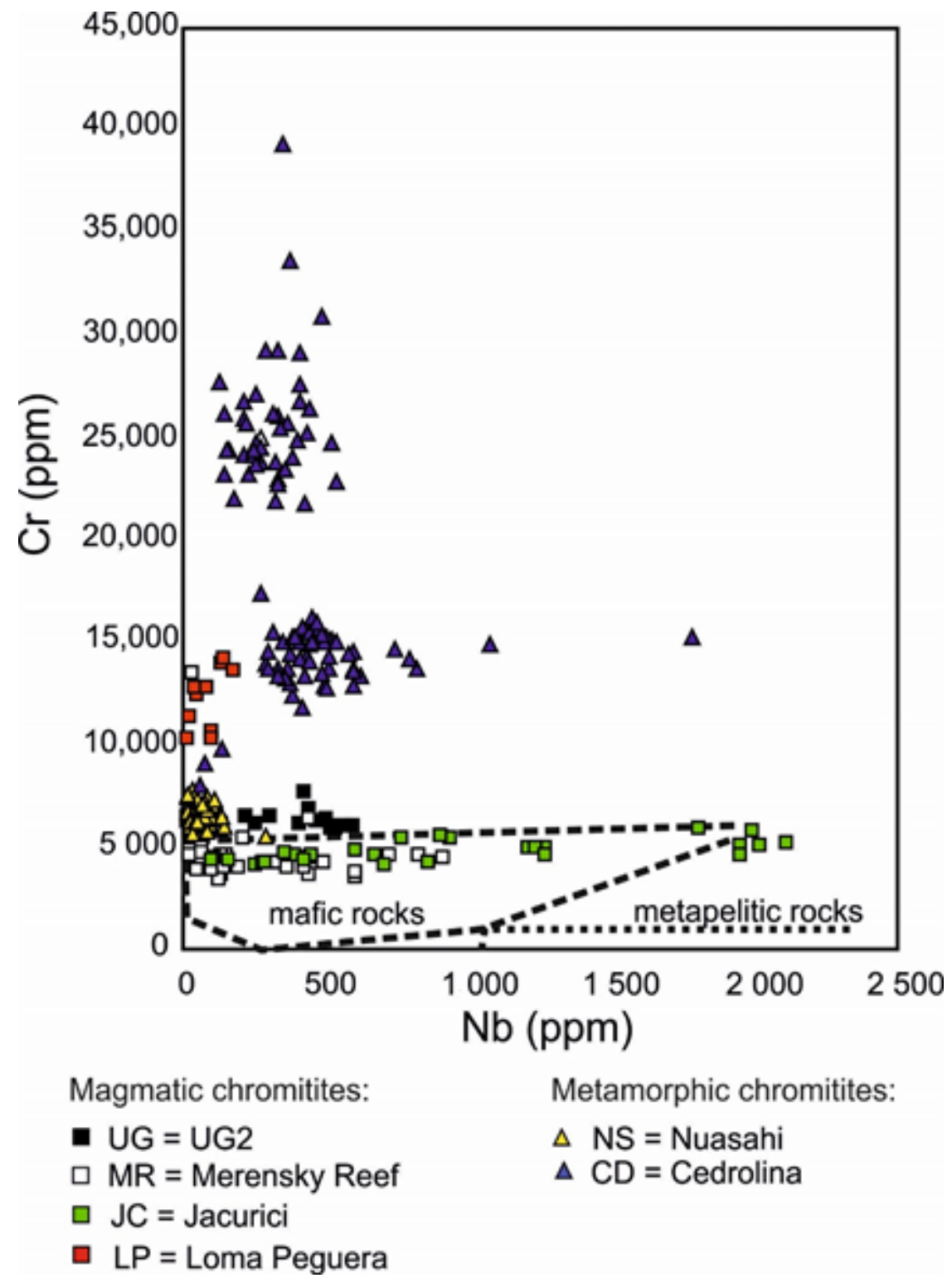

Figure 16. Comparison of $\mathrm{Nb} / \mathrm{Cr}$ relationships in chromitite-rutile with the field of rutile from mafic and meta-sedimentary rocks which characterize for a consistent $\mathrm{Cr}$ depletion (fields redrawn after [7]). Data from the stratiform chromitites of Merensky Reef and Jacurici show a narrow field of overlapping with rutile from mafic rocks.

Author Contributions: F.Z. and G.G. wrote the article. All the authors revised the paper, conceived the experiments, and provided contributions to the data interpretation. G.L.L. calculated and evaluated application of the temperatures based on the $\mathrm{Zr}$-in-rutile thermometry. The samples were collected and provided by G.G. (Jacurici, Merensky Reef, UG2 and Loma Peguera), F.Z. (Loma Peguera), Y.d.M.P. (Cedrolina), and A.K.S. (Nuasahi). All authors have read and agreed to the published version of the manuscript.

Funding: This research received no external funding.

Data Availability Statement: Data are contained in the tables of the article.

Acknowledgments: The authors acknowledge the University Centrum for Applied Geosciences (UCAG) for the access to the E. F. Stumpfl electron microprobe laboratory. The author G.G. thanks the Ministero della Pubblica Istruzione and Consiglio Nazionale delle Ricerche (Rome, Italy) for having financed participation in the Third International Platinum Symposium, South Africa (1981), and field 
work in Brazil (1986-1989). The Ferbasa Company is gratefully acknowledged for the permission to visit the Jacurici mining sites (August 1988, September 1989). The authors G.G. and F.Z. acknowledge the 18va Conferencia Geologica del Caribe, Republica Dominicana, for having organized the field trip to the chromitite occurrences of Loma Peguera (March 2008). We are grateful to the academic editor and two anonymous referees for their fruitful comments. The editorial staff of Minerals is thanked for the help in editing the manuscript.

Conflicts of Interest: The authors declare no conflict of interest.

\section{References}

1. Meinhold, G. Rutile and its applications in earth sciences. Earth Sci. Rev. 2010, 102, 1-28. [CrossRef]

2. Zack, T.; Kooijman, E. Petrology and Geochronology of Rutile. Rev. Mineral. Geochem. 2017, 83, 443-467. [CrossRef]

3. Meinhold, G.; Anders, B.; Kostopoulos, D.; Reischmann, T. Rutile chemistry and thermometry as provenance indicator: An example from Chios Island, Greece. Sedimen. Geol. 2008, 203, 98-111. [CrossRef]

4. Ferreira Filho, C.F.; Kamo, S.L.; Fuck, R.A.; Krogh, T.E.; Naldrett, A.J. Zircon and rutile U-Pb geochronology of the Niquelandia layered mafic and ultramafic intrusion, Brazil: Constraints for detailing of magmatism and high grade metamorphism. Precambrian Res. 1994, 68, 241-255. [CrossRef]

5. $\quad$ Rudnick, R.L.; Barth, M.; Horn, I.; McDonough, W.F. Rutile-bearing refractory eclogites: Missing link between continents and depleted mantle. Science 2000, 287, 278-281. [CrossRef]

6. Zack, T.; Kronz, A.; Foley, S.F.; Rivers, T. Trace element abundances in rutiles from eclogites and associated garnet mica schists. Chem. Geol. 2002, 184, 97-122. [CrossRef]

7. Zack, T.; Moraes, R.; Kronz, A. Temperature dependence of Zr in rutile: Empirical calibration of a rutile thermometer. Contrib. Mineral. Petrol. 2004, 148, 471-488. [CrossRef]

8. Luvizotto, G.L.; Zack, T. $\mathrm{Nb}$ and $\mathrm{Zr}$ behavior in rutile during high-grade metamorphism and retrogression: An example from the Ivrea Verbano Zone. Chem. Geol. 2009, 261, 303-317. [CrossRef]

9. Luvizotto, G.L.; Zack, T.; Triebold, S.; von Eynatten, H. Rutile occurrence and trace element behavior in medium-grade metasedimentary rocks: Example from the Erzgebirge, Germany. Contrib. Mineral. Petrol. 2009, 97, 233-249. [CrossRef]

10. Schmidt, A.; Weyer, S.; John, T.; Brey, G.P. HFSE systematics of rutile-bearing eclogites: New insights into subduction zone processes and implications for the earth's HFSE budget. Geochim. Cosmochim. Acta 2009, 73, 455-468. [CrossRef]

11. Meyer, M.; John, T.; Brandt, S.; Klemd, R. Trace element composition of rutile and the application of Zr-in-rutile thermometry to UHT metamorphism (Epupa Complex, NW Namibia). Lithos 2011, 126, 388-401. [CrossRef]

12. Tomkins, H.S.; Powell, R.; Ellis, D.J. The pressure dependence of the zirconium-in rutile thermometer. J. Metam. Geol. 2007, 25, 703-713. [CrossRef]

13. Portella, Y.D.M.; Zaccarini, F.; Luvizotto, G.L.; Garuti, G.; Bakker, R.J.; Angeli, N.; Thalhammer, O. The Cedrolina Chromitite, Goiás State, Brazil: A Metamorphic Puzzle. Minerals 2016, 6, 91. [CrossRef]

14. Ver Hoeve, T.J.; Scoates, J.S.; Wall, C.J.; Weis, D.; Amini, M. A temperature-composition framework for crystallization of fractionated interstitial melt in the Bushveld Complex from trace element systematics of zircon and rutile. J. Petrol. 2018, 59, 1383-1416. [CrossRef]

15. Cameron, E.N. Titanium-bearing oxide minerals of the Critical Zone of the Eastern Bushveld Complex. Am. Mineral. 1979, 64, 140-150.

16. Scoates, J.S.; Friedman, R.M. Precise age of the platiniferous Merensky Reef, Bushveld Complex, South Africa, by the U-Pb zircon chemical abrasion ID-TIMS technique. Econ. Geol. 2008, 103, 465-471. [CrossRef]

17. Garuti, G.; Zaccarini, F.; Proenza, J.A.; Thalhammer, O.A.R.; Angeli, N. Platinum-Group Minerals in Chromitites of the Niquelândia Layered Intrusion (Central Goias, Brazil): Their Magmatic Origin and Low-Temperature Reworking during Serpentinization and Lateritic Weathering. Minerals 2012, 2, 365-384. [CrossRef]

18. Sunder Raju, P.V. Textural and compositional relationships of rutile and chromite in Sittampundi anorthosite complex, Tamil Nadu. J. Geol. Soc. India 2013, 81, 709-712. [CrossRef]

19. Vukmanovic, Z.; Barnes, S.J.; Reddy, S.M.; Godel, B.; Fiorentini, M.L. Morphology and microstructure of chromite crystals in chromitites from the Merensky Reef (Bushveld Complex, South Africa). Contrib. Mineral. Petrol. 2013, 165, 1031-1050. [CrossRef]

20. Junge, M.; Oberthür, T.; Melcher, F. Cryptic variation of chromite chemistry, platinum-group-element and -mineral distribution in the UG-2 chromitite: An example from the Karee Mine, western Bushveld Complex, South Africa. Econ. Geol. 2014, 109, 795-810. [CrossRef]

21. Scoates, J.S.; Wall, C.J. Geochronology of layered intrusions. In Layered Intrusions; Charlier, B., Namu, O., Latypov, R., Tegner, C., Eds.; Springer Science: Dordrecht, The Netherlands, 2015; pp. 3-74.

22. Friedrich, B.M.; Marques, J.C.; Olivo, G.R.; Frantz, J.C.; Joy, B.; Queiroz, W.J.A. Petrogenesis of the massive chromitite layer from the Jacurici Complex, Brazil: Evidence from inclusions in chromite. Miner. Depos. 2020, 55, 1105-1126. [CrossRef]

23. Zaccarini, F.; Singh, A.K.; Garuti, G.; Zsatyanarayanan, M. Platinum-group minerals (PGM) in the chromitite from the Nuasahi massif, eastern India: Further findings and implications for their origin. Eur. J. Mineral. 2017, 29, 571-584. [CrossRef]

24. Cawthorn, R.G.; Lee, C.A.; Schouwstra, R.P.; Mellowship, P. Relationship between PGE and PGM in the Bushveld Complex. Can. Mineral. 2002, 40, 311-328. [CrossRef] 
25. Cawthorn, R.G.; Merkle, R.K.W.; Viljoen, M.J. Platinum-Group Element Deposits in the Bushveld Complex, South Africa. In The Geology, Geochemistry, Mineralogy and Mineral Benefication of Platinum-Group Elements; Cabri, L.J., Ed.; Canadian Institute of Mining, Metallurgy and Petroleum: Montreal, QC, Canada, 2002; Volume 54, pp. 389-429.

26. Vermaak, C.F.; Von Gruenewaldt, G. Third International Platinum Symposium, Excursion Guidebook; Geological Society of South Africa and Society of Economic Geology: Johannesburg, South Africa, 1981; pp. 1-81. ISBN 0798821715.

27. Naldrett, A.J. Magmatic Sulfide Deposits, Geology, Geochemistry and Exploration; Springer: Berlin/Heidelberg, Germany, $2004 ;$ p. 727.

28. Ballhaus, C.G.; Stumpfl, E.F. Sulfide and platinum mineralization in the Merensky Reef: Evidence from hydrous silicates and fluid inclusions. Contrib. Mineral. Petrol. 1986, 94, 193-204. [CrossRef]

29. Mathez, E.A. Magmatic metasomatism and formation of the Merensky Reef, Bushveld Complex. Contrib. Mineral. Petrol. 1995, 19, 277-286. [CrossRef]

30. Li, C.; Ripley, E.M.; Sarkar, A.; Shin, D.; Maier, W.D. Origin of phlogopite-orthopyroxene inclusions in chromites from the Merensky Reef of the Bushveld Complex, South Africa. Contrib. Mineral. Petrol. 2005, 150, 119-130. [CrossRef]

31. Alkmim, F.F.; Marshak, S.; Fonseca, M.A. Assembling West Gondwana in the Neoproterozoic: Clues from the São Francisco craton region, Brazil. Geology 2001, 29, 319-322. [CrossRef]

32. Marques, J.C.; Ferreira Filho, C.F. The chromite deposit of the Ipueira-Medrado Sill, São Francisco Craton, Bahia State, Brazil. Econ. Geol. 2003, 98, 87-108. [CrossRef]

33. Marques, J.C.; Dias, J.R.V.P.; Friedrich, B.M.; Frantz, J.C.; Queiroz, W.J.A.; Botelho, N. F Thick Chromitite of the Jacurici Complex (NE Craton São Francisco, Brazil): Cumulate chromite slurry in a conduit. Ore Geol. Rev. 2017, 90, 131-147. [CrossRef]

34. Oliveira, E.P.; Carvalho, M.J.; McNaughton, N.J.; Pimentel, M.; Flechter, I.R. Contrasting copper and chromium metallogenic evolution of terranes in the Paleoproterozoic Itabuna-Salvador-Curaçá orogen, São Francisco Craton, Brazil: New zircon (SHRIMP) and Sm-Nd (model) ages and their significances for orogen-parallel escape tectonics. Precambrian Res. 2004, 128, 143-165. [CrossRef]

35. Barbosa de Deus, P.; Moura Marinho, M.; Melo Fagundes Rocha, G.; Dos Santos Viana, J. Chromite potential of the Vale do Jacurici. In Proceedings of the INFACON 86: 4th International Ferro-Alloys Congress, Rio de Janeiro, Brazil, 31 August-3 September 1986; pp. 249-262.

36. Marques, J.C.; Ferreira Filho, C.F.; Carlson, R.W.; Pimentel, M.M. Re-Os and Sm-Nd isotope and trace element constraints on the origin of the chromite deposit of the Ipueira-Medrado Sill, Bahia, Brazil. J. Petrol. 2003, 44, 659-678. [CrossRef]

37. Ferreira Filho, C.F.; Araújo, S.M. Review of Brazilian chromite deposits associated with layered intrusions: Geological and petrological constraint for the origin of stratiform chromitites. Appl. Earth Sci. TIMM B 2009, 118, 86-100. [CrossRef]

38. Draper, G.; Gutiérrez, G.; Lewis, J.F. Thrust emplacement of the Hispaniola peridotite belt: Orogenic expression of the midCretaceous Caribbean arc polar reversal? Geology 1996, 24, 1143-1146. [CrossRef]

39. Dilek, Y.; Furnes, H. Ophiolite genesis and global tectonics: Geochemical and tectonic fingerprinting of ancient oceanic lithosphere. Geol. Soc. Am. Bull. 2011, 123, 387-411. [CrossRef]

40. Kerr, A.C.; Pearson, D.G.; Nowell, G.M. Magma source evolution beneath the Caribbean oceanic plateau: New insights from elemental and Sr-Nd-Pb-Hf isotopic studies of ODP Leg 165 Site 1001 basalts. In The Origin and Evolution of the Caribbean Plate; James, K.H., Lorente, M.A., Pindell, J.L., Eds.; Geological Society: London, UK, 2009; Volume 328, pp. 809-827.

41. Proenza, J.A.; Zaccarini, F.; Lewis, J.F.; Garuti, G.; Longo, F. Platinum Group Element distribution and mineralogy in Loma Peguera chromitites, Loma Caribe peridotite, Dominican Republic. Can. Mineral. 2007, 45, 631-648. [CrossRef]

42. Zaccarini, F.; Bindi, L.; Garuti, G.; Proenza, J. Ruthenium and magnetite intergrowths from the Loma Peguera chromitite, Dominican Republic, and relevance to the debate over the existence of platinum-group element oxides and hydroxides. Can. Mineral. 2014, 52, 617-624. [CrossRef]

43. Lewis, J.F.; Draper, G.; Proenza, J.A.; Espaillat, J.; Jimenez, J. Ophiolite-related ultramafic rocks (serpentinites) in the Caribbean region: A review of their occurrence, composition, origin, emplacement and Ni-laterite soils. Geol. Acta 2006, 4, $237-263$.

44. Proenza, J.A.; Lewis, J.F.; Galı', S.; Tauler, E.; Labrador, M.; Melgarejo, J.C.; Longo, F.; Bloise, G. Garnierite mineralization from Falcondo Ni-laterite deposit (Dominican Republic). Macla 2008, 9, 197-198.

45. Mishra, S.; Domuarari, M.P.; Wiedenbeck, M.; Goswami, J.N.; Ray, S.; Saha, A.K. 207Pb/206Pb zircon ages and the evolution of the Singhbhum Craton, eastern India: An ion microprobe study. Precambrian Res. 1999, 93, 139-151. [CrossRef]

46. Saha, A.K. Crustal evolution of Singhbhum North Orissa, Eastern India. Geol. Soc. India Mem. 1994, $27,341$.

47. Augé, T.; Cocherie, A.; Genna, A.; Armstrong, R.; Guerrot, C.; Mukherjee, M.M.; Patra, R.N. Age of the Baula PGE mineralization (Orissa, India) and its implications concerning the Singhbhum Archaean nucleus. Precambrian Res. 2003, 121, 85-101. [CrossRef]

48. Mondal, S.K.; Baidya, T.K.; Rao, K.N.G.; Glascock, M.D. PGE and Ag mineralization in Breccia zone of the Precambrian Nuasahi ultramafic-mafic complex, Orissa India. Can. Mineral. 2001, 39, 979-996. [CrossRef]

49. Das, A.K.; Khaoash, S.; Mishra, P.; Mohapatra, B.K.; Mohanty, J. Chromite-bearing quartzite in the southern fringe of Singhbhum Craton around Ghutrigaon, Eastern India: Petrogenetic implication. Geol. J. 2021, 1-25. [CrossRef]

50. Jost, H.; Fuck, R.; Brod, J.A.; Dantas, E.L.; Meneses, P.R.; Assad, M.L.; Pimentel, M.M.; Blum, M.L.B.; Silva, A.; Spigolon, A.L.D. Geologia de terrenos Arqueanos e Proterozóicos da região de Crixás-Cedrolina, Goiás. Rev. Brasil. Geoci. 2001, 31, 315-328. (In Portuguese) [CrossRef]

51. Portella, Y.D.M.; Zaccarini, F.; Etiope, G. First Detection of Methane within Chromitites of an Archaean-Paleoproterozoic Greenstone Belt in Brazil. Minerals 2019, 9, 256. [CrossRef] 
52. Zaccarini, F.; Yuri de, M.; Portella, Y.; Bakker, R.J.; Angeli, N.; Garuti, G.; Thalhammer, O.A.R. Electron microprobe and Raman spectroscopic investigation of monazite from chromitites of Cedrolina, (Goias State, Brazil). Neues J. Mineral. 2012, 189, 207-215. [CrossRef]

53. Barnes, S.J.; Roeder, P.L. The range of spinel composition in terrestrial mafic and ultramafic rocks. Contrib. Mineral. Petrol. 2001, 42, 2279-2302. [CrossRef]

54. Irvine, T.N. Chromite crystallization in the join $\mathrm{Mg}_{2} \mathrm{SiO}_{4}-\mathrm{CaMgSi}_{2} \mathrm{O}_{6}-\mathrm{CaAl}_{2} \mathrm{Si}_{2} \mathrm{O}_{8}-\mathrm{MgCr}_{2} \mathrm{O}_{4}-\mathrm{SiO}_{2}$. Carn. Inst. Wash. Yearbook $1977,76,465-472$.

55. Latypov, R.; Chistyakova, S.; Page, A.; Hornsey, R. Field evidence for the in-situ crystallization of the Merensky Reef. J. Petrol. 2015, 56, 2341-2372. [CrossRef]

56. Garuti, G.; Proenza, J.A.; Zaccarini, F. Distribution and mineralogy of platinum-group elements in altered chromitites of the Campo Formoso layered intrusion (Bahia State, Brazil): Control by magmatic and hydrothermal processes. Mineral. Petrol. 2007, 89, 159-188. [CrossRef]

57. Garuti, G.; Pushkarev, E.V.; Thalhammer, O.A.R.; Zaccarini, F. Chromitites of the Urals (part 1): Overview of chromite mineral chemistry and geo-tectonic setting. Ofioliti 2012, 37, 27-53.

58. Evans, B.W.; Frost, B.R. Chrome-spinel in progressive metamorphism: A preliminary analysis. Geochim. Cosmochim. Acta 1975, 39, 959-972. [CrossRef]

59. Mansur, E.; Ferreira Filho, C. Chromitites from the Luanga Complex, Carajás, Brazil: Stratigraphic distribution and clues to processes leading to post-magmatic alteration. Ore Geol. Rev. 2017, 90, 110-130. [CrossRef]

60. Zaccarini, F.; Garuti, G.; Cawthorn, G. Platinum group minerals in chromitites xenoliths from the ultramafic pipes of Onverwacht and Tweefontein (Bushveld Complex). Can. Mineral. 2002, 40, 481-497. [CrossRef]

61. Zaccarini, F.; Garuti, G.; Proenza, J.A.; Longo, F. Magmatic platinum-group minerals (PGM) in the Loma Peguera chromitites (Dominican Republic): Further finding. Macla 2011, 15, 203-204.

62. Reznitsky, L.Z.; Sklyarov, E.V.; Suvorova, L.F.; Barash, I.G.; Karmanov, N.S. V-Cr-Nb-W-Bearing rutile in metamorphic rocks of the Slyudyanka Complex, Southern Baikal Region. Geol. Ore Depos. 2017, 59, 1-13. [CrossRef]

63. Zaccarini, F.; Garuti, G.; Pushkarev, E.; Thalhammer, O. Origin of Platinum Group Minerals (PGM) Inclusions in Chromite Deposits of the Urals. Minerals 2018, 8, 379. [CrossRef]

64. Cooper, R.W.; Garuti, G.; Zaccarini, F. Platinum Group Minerals distribution in chromitite layers of the Stillwater complex (Monatana, USA). In Platinum-Group Elements: From Genesis to Benefication and Environmental Impact.; Törmänen, T.O., Alapieti, T.T., Eds.; Geological Survey Finland: Oulu, Finland, 2005; pp. 58-61.

65. Prichard, H.M.; Barnes, S.J.; Fisher, P.C.; Page, P.; Zientek, M.L. Laurite and associated PGM in the Stillwater chromitites: Implications for processes of formation, and comparisons with laurite in the Bushveld and ophiolitic chromitites. Can. Mineral. 2017, 55, 121-144. [CrossRef] 